\title{
White matter integrity of the frontal brain and its relevance for catatonia and executive function
}

\author{
Dissertation \\ for the award of the degree \\ "Doctor rerum naturalium" (Dr.rer.nat.) \\ of the Georg-August-Universität Göttingen
}

within the doctoral program Center for Systems Neuroscience (CSN)

of the Göttingen Graduate School for Neurosciences,

Biophysics and Molecular Biosciences (GGNB)

and of the Georg-August University School of Science (GAUSS)

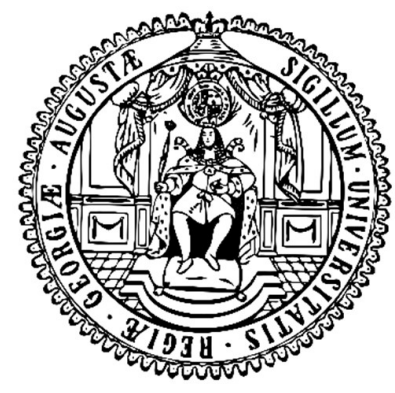

submitted by

\section{Sahab Arinrad}

born in Tehran, Iran

Göttingen, 2020 


\section{Doctoral thesis committee:}

Prof. Dr. Dr. Hannelore Ehrenreich (Supervision, $1^{\text {st }}$ referee)

Clinical Neuroscience

Max Planck Institute of Experimental Medicine

Prof. Dr. Susann Boretius ( $2^{\text {nd }}$ referee)

Functional Imaging Laboratory

German Primate Center

Prof. Dr. Ralf Heinrich

Department of Cellular Neurobiology

Schwann-Schleiden Research Center

PD Dr. Hauke B. Werner

Department of Neurogenetics

Max Planck Institute of Experimental Medicine

\section{Members of the Examination Board}

Prof. Klaus-Armin Nave, Ph.D.

Department of Neurogenetics

Max Planck Institute of Experimental Medicine

\section{Prof. Dr. Tiago Fleming Outeiro}

Department of Experimental Neurodegeneration and Restaurative Research

University Medical Center Göttingen

Prof. Dr. Walter Paulus

Department of Clinical Neurophysiology

University Medical Center Göttingen

Date of the thesis submission: September $30^{\text {th }}, 2020$

Date of the oral examination: November $23^{\text {rd }}, 2020$ 


\section{Declaration}

I hereby declare that the thesis "White matter integrity of the frontal brain and its relevance for catatonia and executive function" has been written independently by myself and without any other sources and aids than cited.

The second chapter of this thesis is adapted from the manuscript "Microglia ablation alleviated myelin-associated catatonic signs in mice", published in the Journal of Clinical Investigation in 2018. I have been given consent from the Journal of Clinical Investigation to incorporate the manuscript into my thesis. The third chapter of this thesis is adapted from the manuscript "Isolated catatonia-executive dysfunction complex in aged mice induced by forebrain-specific loss of myelin integrity", prepared in collaboration with PD Dr. Hauke B. Werner and Prof. Klaus-Armin Nave, Ph.D., from the Department of Neurogenetics at the Max Planck Institute of Experimental Medicine in Göttingen. This manuscript has been submitted and is currently under revision. I have been given consent from all co- and corresponding authors of the manuscript to incorporate it into my thesis.

Sahab Arinrad

Göttingen, September 30th, 2020 


\section{Acknowledgements}

The first person I would like to express my thanks and my appreciation to is my supervisor Prof. Dr. Dr. Hannelore Ehrenreich. I am thankful to have had the opportunity to conduct clinical neuroscientific research under your guidance and thereby to be encouraged to interact with a broad variety of excellent colleagues, all of which were a pleasure to work with. Being part of this multidisciplinary team enabled me to learn and discuss experimental ideas and acquired data ranging from cellular and molecular approaches via mouse models and behavior up to translation into men and clinical subjects, and vice versa. Personally, I am convinced that clinical neuroscience today requires the combination of all these distinct fields of research and I am grateful to have been part of this team. Moreover, throughout my studies I was lucky, not only to interact but further to collaborate scientifically with excellent colleagues such as Prof. Dr. Susann Boretius, Prof. Dr. Ralf Heinrich, PD Dr. Hauke B. Werner and Prof. Dr. KlausArmin Nave. In the framework of my studies, I always felt well supported, equally well advised and I am grateful for all the scientific input you all kindly provided and collaborative output we achieved together. Likewise, I want to express my appreciation to the members of my examination board, Prof. Dr. Tiago Fleming Outeiro and Prof. Dr. Walter Paulus for your kind consideration, and the time you invested into the evaluation of the extent of my scientific contributions.

I always felt welcomed and supported in our lab. Having been to various groups before, I can tell you all, the level of care and support present in our group is by far not common. I want to thank all of you and I will do so in person in due time. However, one member has to be mentioned here in specific. Anja, from day one we got along like a well-established team and by now we've grown into lifelong friends. I cannot express how much I value you and I am humbled to have been given the opportunity to learn all there is to know with respect to educated and proper behavioral neuroscience from you. Additionally, I have to thank Dr. Jan Seidel and Dr. Umer Javed Butt. You guys know exactly what I am grateful for. Friends like the two of you are rare to find.

Lastly, I just want to say that I sincerely hope I was able to contribute to the team, the institute and maybe even to our collaborators throughout the last years. At least I always aimed to do so. Moreover, I am grateful to have had this experience in my hometown; this really meant a lot to me. 


\begin{abstract}
Catatonia is a CNS derived psychomotor syndrome comprising disturbed volition and aberrant motor and behavioral features. Targeted and effective treatment today is scarce and further impeded by its heterogeneous clinical representation across various CNS disorders. Moreover, respective research on the etiology and underlying cellular pathomechanisms are hampered by sustained conceptual limitations, inadequate clinical rating scales and the lack of reliable animal models.

To this end, we had previously reported a catatonia-like phenotype in C57Bl/6 mice heterozygous for the major myelin genes $C n p$ and $M b p$ upon progressed age, along with indications of low-grade neuroinflammation. In the first project of my thesis, I thus addressed the question whether neuroinflammation of subcortical white matter is causative to catatonia in both mice and man. Neurological assessment of schizophrenic subjects $(n=1095)$ revealed a high prevalence of catatonic signs $(25 \%)$, which were more pronounced in carriers of a CNP loss-of-sunction SNP (rs207106-AA). Additionally, elevated signs of white matter hyperintensities were observed in carriers of the SNP in a general population sample by neuroimaging. Cnp-null mutant mice exhibit catatonic signs as early as 8 weeks of age. Importantly, microglia targeted treatment via the CSF1 receptor inhibitor PLX5622, successfully prevented the occurrence of the phenotype upon early treatment and further alleviated catatonic signs even at progressed age. The beneficial impact of PLX5622 on mouse behavior was accompanied by sustained reduction of neuropathology, i.e. microgliosis and neurodegeneration. Collectively these findings indeed suggest key involvement of impaired white matter integriy and neuroinflammation in the etiology of catatonia.

Based on a follow-up study, which revealed a strong correlation of catatonia and executive dysfunction in mice and man, the objective of the second project was to determine the relevance of white matter integrity of the frontal brain in the etiology of the psychomotor syndrome. A novel mouseline, lacking the major myelin gene $P / p 1$ in $E m x 1$ expressing ventricular zone stem cells of the forebrain (cKO), was characterized on a behavioral and neuropathological scope. Longitudinal and elaborate behavioral assessment revealed an isolated catatonia-executive dysfunction in cKO mice of both genders, while no other behavioral domain was affected. Neuropathology revealed significant astro- and microgliosis along with neurodegeneration, exclusively in frontal strucutres such as the fimbria and corpus callosum, thereby confirming the crucial importance of white matter integrity of the frontal brain in the observed catatonia-like phenotype in the here reported mouse models.
\end{abstract}




\section{Table of contents}

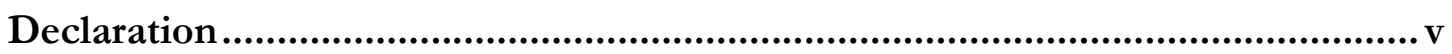

Acknowledgements..................................................................................................vii

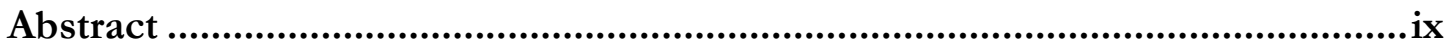

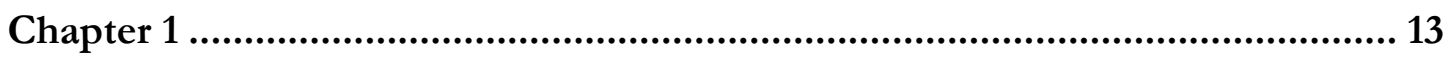

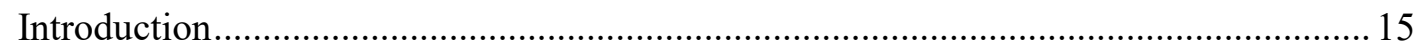

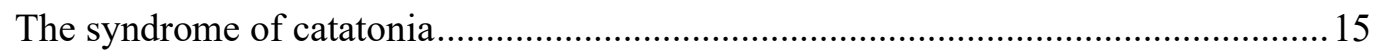

Myelination in health and neuropsychiatric conditions ............................................. 19

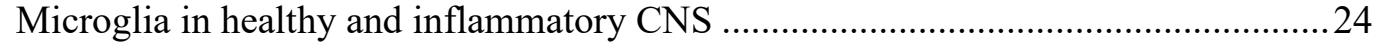

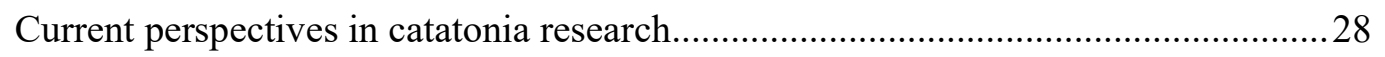

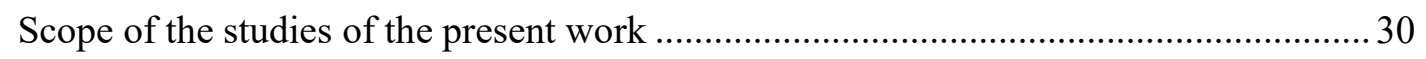

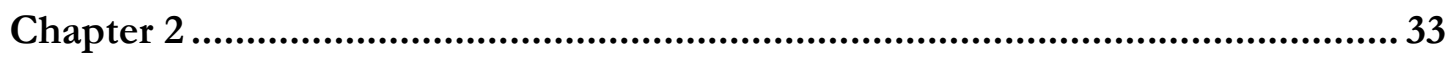

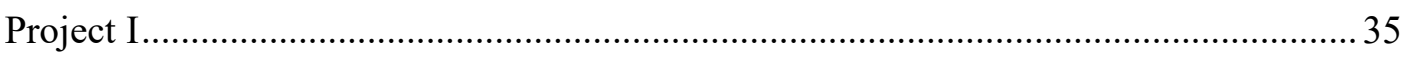

Microglia ablation alleviates myelin-associated catatonic signs in mice ......................35

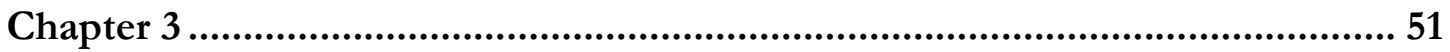

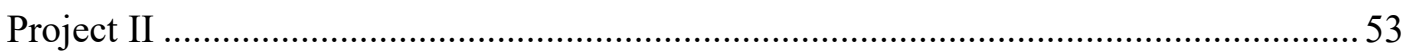

Isolated catatonia-executive dysfunction complex in aged mice induced by forebrainspecific loss of myelin integrity ............................................................................ 53

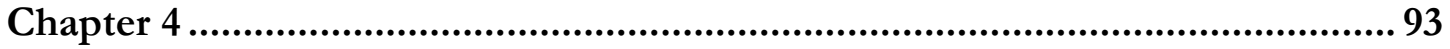

Conclusions and Future Perspectives ...........................................................................95

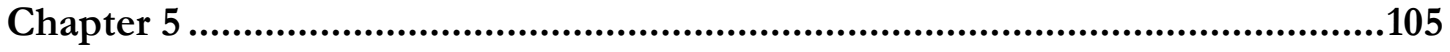

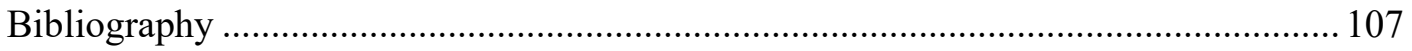

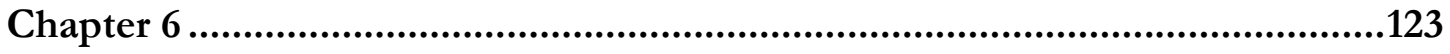

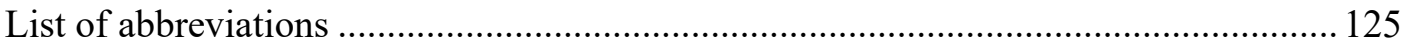

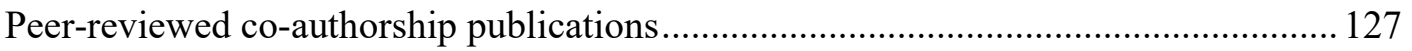

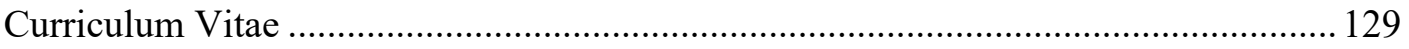


Chapter 1

\section{Introduction}




\section{Introduction}

\section{The syndrome of catatonia}

The first report on the syndrome of catatonia dates back to the year 1874. The German physician Karl Ludwig Kahlbaum reported on observations of patients exhibiting hypo- and hyperkinetic motor and behavioral abnormalities along with affective symptoms, which he characterized as the independent psychomotor syndrome catatonia (Kahlbaum, 1874, 2007). Specifically, catatonic patients present with disturbed volition, resulting in distinct motor and behavioral features, such as rigor, posturing, counteracting or catalepsy as well as affective symptoms like anxiety or flat affect (Hirjak et al., 2020). Collectively, all three symptom domains result in aberrant psychomotor behavior, which can be both increased or decreased, likely dependent on the given context (Walther et al., 2019).

In contrast to this initial characterization, catatonia has historically been considered a subtype of dementia praecox, as described by Emil Kraepelin (1899), thereby diminishing its acknowledgement as an independent diagnostic syndrome. Eugen Bleuler later on introduced the concept of schizophrenia (1911), in which he classified catatonia as a subtype. It was thereby further neglected as a separate entity, until the removal of all schizophrenia subtypes in the Diagnostic and Statistical Manual of Mental Disorders 5 (DSM-5) in 2013 (American Psychiatric Association, 2013; Walther et al., 2019). However, as defined by DSM-5 and the International Statistical Classification of Diseases 10 (ICD-10; American Psychiatric Association, 2013; World Health Organization, 2004), catatonia remains unacknowledged as an independent diagnostic entity to date. Due to these tight limitations it was mostly recognized by its motor and behavioral features only, thereby neglecting the affective component as originally described by Kahlbaum. Consequently, clinical examinations focused particularly on behavioral and motor abnormalities, of which DSM-5 defines a total of 12 as catatonic symptoms (American Psychiatric Association, 2013). Likewise, the application of many respective diagnostic rating scales, such as the Modified Rogers Scale, the Bräunig Catatonia Rating Scale or the Bush-Francis Catatonia Rating Scale (Bräunig et al., 2000; Bush et al., 1996a, 1996b; Lund et al., 1991; McKenna et al., 1991), unfortunately neglect the important factor of affective symptoms. Taken together, these shortcomings are rendering catatonia essentially misperceived and underepresented in scientific research.

This misconception of catatonia (Appiani et al., 2018), fueled by the failure of examiners to assess signs or their misinterpretation (Van der Heijden et al., 2005), ultimately impairs the 


\section{Introduction}

distinction of catatonia from other medical conditions. In addition, catatonia has a high comorbidity with various medical conditions, and its features are observed across numerous neuropsychiatric disorders such as schizophrenia, mood disorders, autism spectrum disorders (ASD) and affective psychosis (Hirjak et al., 2020; Walther et al., 2019). Inflammatory and immunological conditions such as anti- $N$-methyl-D-aspartate receptor (NMDAR) encephalitis or multiple sclerosis (MS) have also been associated with catatonic features (Rogers et al., 2019). In summary, lack of experience by examiners along with rating scales, which often neglect affective symptoms, and its high comorbidity, are rendering catatonia underdiagnosed. Moreover, the prominent psychomotor symptoms, nowadays used to define catatonia, are observed across various medical conditions, ultimately hampering research, diagnosis and therapy. Walther et al. (2019), therefore, recently suggested future studies to evaluate differences in the clinical presentation, quality and the possibly underlying mechanisms of these symptoms throughout different disease conditions, to improve both diagnosis and treatment of catatonia.

Therapeutical approaches specifically rely on mechanistic insight, allowing for improved understanding of the underlying neuroanatomical and biochemical causes of catatonia. In this regard, an abundance of neuroimaging studies on human subjects have been conducted, collectivly aiming to uncover and delineate underlying neural mechanisms and involved central nervous circuits (Iseki et al., 2009; Northoff, 2000; Northoff et al., 2004; Scheuerecker et al., 2009). Depending on the diagnostic rating scales applied, as well as the central symptom domain considered thereby, the current state of research is associating catatonia to alterations and dysfunctions of either cortico-subcortical circuits or higher-order fronto-parietal networks (Hirjak et al., 2020; Northoff, 2000). Although both concepts share the involvement of motor circuits, such as the primary motor cortex and the supplementary motor area, they differ with regard to the importance of other interconnected brain regions. Studies focusing on corticosubcortical circuits suggest a critical involvement of the thalamus and the cerebellum and find mainly dopaminergic neurotransmission to be causative to catatonia (Hirjak et al., 2020; Northoff et al., 1995; Walther et al., 2017). The higher-order fronto-parietal concept of catatonia, however, implies key involvements of the parietal and prefrontal cortex (PFC) and reports altered gamma-aminobutoric acid (GABA) transmission to be crucial in the etiology of catatonia (Northoff, Braus, et al., 1999; Richter et al., 2010; Ungvari et al., 1994). 
Despite the advantages of state-of-the-art research and imaging techniques, the etiology and underlying cellular pathomechanisms of catatonia remain poorly understood. Consequently, adequate and efficient treatment are scarce and a challenge to date. The most commonly used approaches are electroconvulsive therapy (ECT) and medication targeting GABAergic neurotransmission, such as benzodiazepines (American Psychiatric Association, 2013; Bush et al., 1996b; Girish et al., 2003; Northoff, Braus, et al., 1999; Northoff et al., 1995; Seethalakshmi et al., 2008). However, both approaches are of unspecific nature and the effectiveness of benzodiazepines depends on various factors, including chronicity and presence or absence of psychosis (Bush et al., 1996b; Ungvari et al., 1994; Ungvari et al., 1999). Clinical presentation of catatonia is heterogeneous and may show along with other complications such as renal failure, pulmonary thrombosis, delirium or psychosis. Antipsychotic treatment of catatonic patients has been reported (Numata et al., 2001; Valevski et al., 2001; Van Den Eede et al., 2005), although their frequent usage is controversial (Bahro et al., 1999; Markham-Abedi et al., 2007). In fact, antipsychotic treatment, by dopamine $\mathrm{D}_{2}$ receptor antagonists, may induce or worsen catatonia (Cassidy et al., 2001; Graham et al., 2001; Hirjak et al., 2020). Thus, in treating psychotic patients, presenting with catatonic features, alternative treatment with a low affinity for dopamine receptors is suggested (Beach et al., 2017; Madigand et al., 2016; Martényi et al., 2001; Walther et al., 2019).

In summary, research on catatonia has been hampered by various factors. Moreover, translational approaches, by application of animal models, remain challenging to date, due to its heterogeneous symptomatology. However, animal models are crucial to studying the underlying mechanisms in the various clinical presentations of catatonia, rendering them indispensable with respect to the development of potential therapeutic strategies. While the scientific community is essentially lacking a construct-valid rodent model of catatonia to date, recent studies from our group have reported an age-dependent catatonia-depression syndrome in $\mathrm{C} 57 \mathrm{Bl} / 6$ mice upon heterozygous deficiency of the myelin genes $2^{\prime}, 3^{\prime}$-cyclic nucleotide $3^{\prime}$-phosphodiesterase $(C n p)$ and myelin basic protein (Mbp; Hagemeyer et al., 2012; Poggi et al., 2016). In both studies, the authors reported an age-dependent low-grade inflammation of the central nervous system (CNS) along with a catatonia-like phenotype. Interestingly, Hagemeyer et al. (2012) also described a similar age-dependent catatonia-depression syndrome in human subjects suffering from schizophrenia, which carry the loss-of-function single nucleotide polymorphism (SNP) rs2070106. Carriers of this SNP exhibited diminished expression of CNP messenger ribonucleic acid (mRNA). Additionally, diffusion tensor imaging (DTI) revealed signs of increased axonopahty and inflammation in the frontal brain regions of carriers (Hagemeyer et al., 2012). 


\section{Introduction}

Collectivly, these findings indicate a critical role of white matter integrity and neuroinflammation in catatonia and point to altered frontal brain functions. In need of a better understanding of catatonia, its mechanistic causes and involved brain circuits, experimental approaches in elaborate mouse models constitute reasonable and promising measures, ultimately aiming to improve treatment of the syndrome. 


\section{Myelination in health and neuropsychiatric conditions}

Healthy functioning of the CNS and peripheral nervous system, including cognition, motor and sensory functions, require fast and coherent conduction of signals between cortical and subcortical areas and peripheral organs. This rapid propagation of action potentials is provided by myelination, the ensheathment of neuronal axons with multiple concentrical layers of compact myelin, providing electrical insulation. The complex process of myelination by CNS oligodendrocytes has been reported on in great detail before (Bunge, 1968; Hildebrand et al., 1993; Kuhn et al., 2019; Michalski et al., 2015; Nave, 2010; Nave \& Werner, 2014; Simons et al., 2016). Schwann cells or oligodendrocytes carry out this critical task within the PNS or CNS, respectively. However, while both cell types essentially carry out the same functions, they differ with respect to various features including their origin, the process of myelination and the thereby utilized structural proteins (Nave \& Werner, 2014). In the framework of this doctoral thesis, I focused on the white matter integrity of the CNS, knowingly dependent on proper oligodendrocyte function. Alterations, such as deficiency of critical myelin genes, not only impair structure and function of myelin, but are increasingly appreciated as underlying features of neuropsychiatric disorders, as I will discuss below.

Myelination, carried out by mature oligodendrocytes, allows for rapid and efficient saltatory signal propagation (Nave, 2010). Initially, multipolar oligodendrocyte progenitor cells (OPCs), originating from precursor cells of the subventricular zone (SVZ), migrate through white matter tracts and constantly extend and retract their numerous processes in search for unoccupied space (Hughes et al., 2013; Kirby et al., 2006). Unmyelinated axons are enwrapped by concentrical layers of expanded oligodendrocyte membrane, via simultaneous radial and lateral movements (Michalski \& Kothary, 2015; Snaidero et al., 2014). While ensheathment is driven by the uncompacted most inner layer outwards, compaction of myelin is carried out the other way around and crucially relies on the myelin gene Mbp (Aggarwal et al., 2013; Readhead et al., 1990; Roach et al., 1983; Schain et al., 2014; Snaidero et al., 2014). Essentially, MBP encloses the myelin membrane by connecting two opposing bilayers at their cytoplasmic sites (Aggarwal et al., 2013; Nave \& Werner, 2014). By simultaneous interaction with multiple axons, a single oligodendrocyte can generate and maintain numerous internodes (Nave, 2010), thereby facilitating and maintaining healthy CNS function and white matter integrity. 


\section{Introduction}

In addition to myelination, oligodendrocytes further ensure survival of the axons by long-term trophic and metabolic support. Metabolites such as lactate are transported to the cytoplasmic non-compacted sites via monocarboxylate transporters (MCTs), and provide energy supply by mitochondrial production of adenosine triphosphate (ATP; Fünfschilling et al., 2012; Lee et al., 2012; Rinholm et al., 2011; Saab et al., 2013). Structural compounds, required for myelin maintenance, are transported through non-compacted cytosolic channels within compacted myelin (Flores et al., 2008; Narayanan et al., 2009; Nave, 2010). By secretion of respective exosomes, oligodendrocytes even exert neuroprotective functions (Frühbeis et al., 2013). Moreover, they remain adaptive in adulthood and can thus respond appropriately to environmental demands (Baraban et al., 2016; Mount et al., 2017; Young et al., 2013). Gibson et al. (2014) reported proliferation of OPCs and particularly thickening of myelin in response to distinct neuronal activity. In fact, numerous studies have shown myelination to be stimulated upon activity (Bengtsson et al., 2005; Keller et al., 2009; Sampaio-Baptista et al., 2013), while other studies linked diminished myelination of the PFC to social isolation in mice (Liu et al., 2012; Makinodan et al., 2012). Additionally, transgenic mice lacking the ability of de novo myelination exhibited inferior motor performance compared to controls (McKenzie et al., 2014). Taken together, these findings reflect the ability of oligodendrocytes to adapt to environmental demands on multiple levels including number of cells, size of axonal myelin and de novo myelination. Thus, myelinating cells constitute a crucial feature of white matter integrity and the preservation of reliable, plastic and healthy CNS functioning (Hughes et al., 2013; Purger et al., 2016; Sampaio-Baptista et al., 2017; Young et al., 2013).

Insults to this sensitive system may cause or promote CNS disorders, as previously reported by Nave and Ehrenreich (2014) . The authors discussed possible mechanisms, reflecting how altered function of oligodendrocytes may contribute to the development of neuropsychiatric disorders such as schizophrenia, ASD as well as degenerative disorders including Alzheimer's disease (AD) or Huntington's chorea. The hypothesized mechanisms range from hypo- or hypermyelination to the loss of axonal metabolic support. The downstream impact on CNS functioning and cognition likely depending on the initial alteration of oligodendrocyte function (Nave \& Ehrenreich, 2014). Continuous metabolic support of axons, by myelinating oligodendrocytes, is critical to healthy axonal functioning (Nave, 2010) and disruption of energy supply has been associated with cognitive decline (Dean et al., 2017; Mosconi et al., 2009; Winkler et al., 2015), likely due to the formation of spheroids and degenerative processes (Saab et al., 2013). Postmortem analysis of brains of SSD subjects revealed significant reduction of oligodendrocyte transcripts (Hakak et al., 2001). Additionally, diminished white matter integrity 


\section{Introduction}

has been reported in SSD, bipolar disorder and major depression disorder (MDD; Edgar \& Sibille., 2012; Fields, 2008; Roussos \& Haroutunian, 2014). Hence, the structural and functional integrity of white matter is increasingly appreciated in higher-order cognitive abilities and mental conditions particularly in recent years (Filley et al., 2016; Haroutunian et al., 2014; Tomassy et al., 2014). As Haroutunian et al. (2014) reported, each of these conditions are known to be multifactorial, and while some are linked to developmental alterations, others unfold throughout adulthood and can be facilitated by the process of aging. The latter is known to affect white matter integrity and cognitive abilities such as memory (Bowley et al., 2010; Lasiene et al., 2009; Peters, 2002; Sturrock, 1976; Wang et al., 2020). However, disruption of white matter integrity is likely a consequence of neuronal loss, triggering dys- or demyelination and eventually gliosis and inflammation of the CNS (Nave \& Ehrenreich, 2014).

To evaluate the relevance of the above-mentioned hypothesis in the context of my doctoral studies, I focused on the consequences of $C N P / C n p$ and proteolipid protein $(P L P / P / p)$ deficiencies. Both are critical myelin genes with individual subsets of features and distinct spatiotemporal expression by oligodendrocytes. The cholesterol-associated transmembrane protein PLP, predominantly expressed by oligodendrocytes (Lüders et al., 2017; Trapp et al., 1987; Werner et al., 2013), is the most abundant protein residing in compact CNS myelin (de Monasterio-Schrader et al., 2012; Jahn et al., 2009; Nave \& Werner, 2014). While PLP and its shorter isoform DM20 are not required for myelin assembly or compaction (Klugmann et al., 1997), they have been shown to be critical to the maintenance and survival of myelinated axons (Griffiths et al., 1998), likely being key players in their proper metabolic support (Hirrlinger et al., 2014; Nave, 2010; Stassart et al., 2018). Moreover, PLP and DM20 are involved in the assembly of cholesterol into CNS myelin (Werner et al., 2013), known to be a rate limiting factor to myelin biogenesis (Nave \& Werner, 2014), as well as the rate of biogenesis itself (de Monasterio-Schrader et al., 2013; Yool et al., 2001). Mutations of PLP are causative to a number of human diseases such as Pelizaeus-Merzbacher disease (PMD) or spastic paraplegia type 2 (SPG2), with similar phenotypes observed in respective mouse models (Anderson et al., 1998;

Griffiths et al., 1998; Saher et al., 2012; Saugier-Veber et al., 1994). Constitutive deficiency $\left(P / p^{\text {null } / Y}\right)$ in mice causes alterations of CNS myelin regarding both its components and ultrastructure (Duncan et al., 1987; Garbern et al., 2002; Klugmann et al., 1997; Möbius et al., 2016; Möbius et al., 2008; Rosenbluth et al., 2006; Werner et al., 2013; Werner et al., 2007) along with impaired axonal transport (Edgar et al., 2004). 


\section{Introduction}

Importantly, $P / p^{\text {mull/Y }}$ mice exhibit progressive axonopathy including axonal spheroids and degeneration throughout the CNS, along with secondary neuroinflammation and gliosis (Edgar et al., 2004; Griffiths et al., 1998; Lüders et al., 2019; Lüders et al., 2017). However, they do not exhibit demyelination (Griffiths et al., 1998), suggesting independent functions of oligodendrocytes with respect to myelination or axonal survival and maintenance (Saab et al., 2013). The most prominent behavioral phenotype of these mice is their inability to perform on the RotaRod at progressed age, reflecting a strong motor impairment along with altered gaiting and spasticity (Griffiths et al., 1998). The severe motor impairments are preceded by other subtle behavioral deficits including increased pain sensitivity, diminished odor perception and particularly impaired executive function in the Puzzle box paradigm, as early as 3 months of age (Gould et al., 2018; Petit et al., 2014). The latter, suggests oligodendrocyte malfunction in higherorder cognitive functions of the frontal lobes and possibly throughout neuropsychiatric conditions per se (Nave \& Ehrenreich, 2014).

In this respect, significant reduction of another important myelin gene, $C N P$, has been reported in postmortem brains of schizophrenic, bipolar and depressive patients (Aston et al., 2005; Mitkus et al., 2008; Tkachev et al., 2003). Like PLP, CNP is abundantly expressed by oligodendrocytes in the brain and not required for myelin assembly but for axonal survival (Lappe-Siefke et al., 2003; Scherer et al., 1994; Yu et al., 1994). Within myelin, CNP is only found in non-compacted regions and counteracts the MBP-driven compaction of myelin (Braun et al., 1988; Gravel et al., 1996; Saab et al., 2013; Scherer et al., 1994; Simons \& Nave, 2016; Yin et al., 1997). CNP provides numerous functions to CNS myelin including involvement in oligodendrocyte process outgrowth, interaction with the cytoskeleton, metabolic support of axons by maintenance of cytosolic channels, and maintenance of axon-glia interactions at nodes of Ranvier (Bauer et al., 2009; Lappe-Siefke et al., 2003; Lee et al., 2005; Rasband et al., 2005; Saab et al., 2013). Constitutive lack of $C_{n} p\left(C_{n}{ }^{-/}\right)$in mice results in progressive neurodegeneration and inflammation throughout the CNS along with reactive gliosis and severe motor dysfunctions, which eventually lead to premature death (Lappe-Siefke et al., 2003). Hence, deficiency of the critical myelin genes Plp and Cnp both result in severe axonopathy and combination of them has recently been shown to enhance pathology (Lüders et al., 2017). Heterozygous deficiency of $\operatorname{Cnp}\left(C_{n} p^{+-}\right)$results in an age-dependent low-grade neuroinflammation, axonal spheroids and a distinct behavioral phenotype reminiscent of the psychomotor syndrome of catatonia (Hagemeyer et al., 2012). Importantly, a similar agedependent catatonia-depression syndrome along with indications of axonopathy was observed in subjects suffering from schizophrenia, carrying a partial CNP loss-of-function SNP 


\section{Introduction}

(Hagemeyer et al., 2012). However, it remains unclear to which extent neuroinflammation contributes to the observed phenotype regarding its development and its progression. Furthermore, we need to evaluate whether this phenotype is exclusive to oligodendrocytespecific alterations. Importantly, the distinct role of higher-order frontal brain circuits remains to be addressed, aiming to evaluate whether catatonia is driven by motor impairments or rather reflects impaired volition and executive functions. 


\section{Introduction}

\section{Microglia in healthy and inflammatory CNS}

The integrity of a healthy CNS further requires intact function and appropriate immune responses by microglia, the resident macrophages of the CNS. These highly dynamic cells are covering a broad array of tasks, ranging from CNS development and homeostasis to responding to environmental demands and insults to the system, including remyelination upon damage (Colonna et al., 2017; Lloyd et al., 2017). Under healthy CNS conditions, subpopulations exhibit characterisitic and region-specific functions along with respective gene expression patterns (Ayata et al., 2018; De Biase et al., 2017; Grabert et al., 2016; Marshall et al., 2014). Microglial functions and morphology may shift to detrimental phenotypes under pathological conditions and thereby facilitate neuroinflammation and -degeneration (Colonna et al., 2017; Tay et al., 2018). Consequently, abberant functions have been shown to be implicated in various developmental, degenerative and psychiatric CNS disorders (Frick et al., 2013; Salter et al., 2017). In this section, I will therefore discuss the origin and function of microglia in health and conclude with implications of microglia in CNS disorders predominantly due to neuroinflammation.

Microglia derive from erythro-myeloid progenitors in the yolk-sac and sustain into adulthood via continuous self-renewal (Ajami et al., 2007; Alliot et al., 1999; Kierdorf et al., 2013; Perdiguero et al., 2015). By rapid proliferation they colonize the entire CNS (Ginhoux et al., 2010; Kierdorf et al., 2013), reaching a steady-state population within weeks after birth (Nikodemova et al., 2015). In this regard, the class III receptor tyrosine kinase colonystimulating factor 1 receptor (CSF1-R) and its natural ligands CSF1 and interleukin-34 (IL-34) are of particular importance, since they are essential to microglial development, proliferation and survival (Chitu et al., 2016 ). Expression of the CSF1-R is not limited to microglia only, but also found on neuronal subpopulations (Murase et al., 1998; Nandi et al., 2012; Wang et al., 1999). Its ligands CSF-1 and IL-34 are predominantly expressed by neurons with distinct spatiotemporal patterns throughout the CNS (Nandi et al., 2012; Wang et al., 2012). Both contribute to the development and steady-state maintenance of microglia and other target cells (Greter et al., 2012; Nandi et al., 2012; Wang et al., 2012; Wei et al., 2010). Deficiency of the CSF1-R and CSF-1 in mice have been shown to affect microglial numbers as well as structure and function of respective CNS sites, largely reflecting neurodegeneration, which can result in behavioral and anatomical alterations (Chitu et al., 2016). 


\section{Introduction}

Separated from the periphery via the blood-brain barrier (BBB), microglia are the innate immune cells of the CNS and crucial to regulating brain homeostasis (Matcovitch-Natan et al., 2016). Along with the developing CNS, they mature into a ramified and motile morphology (Colonna et al., 2017), constantly surveilling and interacting with the environment via their dynamic processes (Davalos et al., 2005; Nimmerjahn et al., 2005). Microglial motility enforces multiple physiological responsibilities at resting state including sculpting neural circuits during development (Schafer et al., 2012), neurogenesis and synaptic pruning and plasticity by diverse measures (Kettenmann et al., 2013; Salter et al., 2016), even in adulthood. Of the various microglial surface receptors, the C-X3-C Motif Chemokine Receptor 1 (CX 3 CR1) is of major importance to microglia-neuron interactions (Eyo et al., 2013; Kierdorf et al., 2017). Moreover, microglia are a part of the innate immune system and respond to CNS insults by exerting tissue repair and anti-inflammatory functions (Colonna et al., 2017; Ransohoff et al., 2016). By promoting oligodendrocyte survival and functions (Miron, 2017), microglia are also involved in myelination and remyelination processes (Hagemeyer et al., 2017; Lampron et al., 2015; Olah et al., 2012; Wlodarczyk et al., 2017). Importantly, remyelination upon damage is a prerequisite of restoring and preserving healthy axonal functions (Irvine et al., 2008; Mei et al., 2016).

Taken together, microglia assist in maintaining CNS integrity by above-mentioned means, though their morphology and funtions can be heterogeneous (Doorn et al., 2015; Grabert et al., 2016; Lawson et al., 1990). Microenvironments, and other signals experienced over time, may distinguish microglial subpopulations (De Biase et al., 2017; Gosselin et al., 2017). Collectivly, microglia ensure CNS maintenance and respond to injury and damage via immune responsive and neuroprotective functions (Glezer et al., 2007, Hickman et al., 2013; Rivest et al., 2009). However, excessive activation by environmental factors (Gosselin et al., 2014; Mosher et al., 2015; Perry et al., 2014), aging (Hickman et al., 2013; Ritzel et al., 2015; Sierra et al., 2007) or CNS disease (Mastroeni et al., 2018; Spittau, 2017) can shift microglial activities into detrimental and pro-inflammatory modifiers (Frick et al., 2013; Tay et al., 2018; Wes et al., 2016). Rather than exerting protective functions, they become highly activated, neurotoxic and fail to exert regular functions in the CNS (Choi et al., 2011; Du et al., 2017; Graeber et al., 2011; Streit et al., 2005). Dependent on the given context, progressive neuroinflammation along with microgliosis, abberant phagocytic activities and demyelination, ultimately contribute to degeneration, which in turn facilitates various CNS disorders (El Khoury, 2010; Frick et al., 2013; Streit et al., 2004; Tay et al., 2018). Microglial dysfunctions are thereby implicated in developmental disorders such as Nasu-Hakola disease(NHD; Bianchin et al., 2004; Paloneva et al., 2001), ASD (Lee et al., 2017; Morgan et al., 2010) or hereditary diffuse leukoencephalopathy with spheroids (HDLS; 


\section{Introduction}

Konno et al., 2014; Rademakers et al., 2012). Moreover, abberant microglial functions are linked to the development of neuropsychiatric disorders including MDD (Holmes et al., 2018; Wohleb et al., 2018), bipolar disorder (Muneer, 2016; Naaldijk et al., 2016) and SSD (Monji et al., 2013; Wierzba-Bobrowicz et al., 2005). Lastly, microglia are also associated with degenerative conditions such as AD or Parkinson’s disease (PD; Doorn et al., 2014; Hong et al., 2016; McGeer et al., 1987; Subramaniam et al., 2017; Wang et al., 2015).

The involvement of microglia in numerous CNS disorders is increasingly investigated, particularly in recent years. However, the question whether microglial dysfunctions are the cause or a mere consequence of other underlying features remains to be addressed. Therapeutic strategies, such as glial replacement (Cartier et al., 2014; Shen et al., 2017; Srivastava et al., 2018), have emerged as promising approaches in that regard. Another frequently used experimental setup involves genetic or pharmaceutical depletion of microglia along with repopulation subsequent to the latter (Han et al., 2019; Waisman et al., 2015). A critical feature of repopulating microglia in restoring CNS health upon damage are their contributions to remyelination (Beckmann et al., 2018; Lloyd et al., 2017). Interestingly, mutations of the CSF1-R in HDLS are associated with progressive atrophy of frontal and parietal white matter along with thinning of the corpus callosum (CC; Kondo et al., 2013; Konno et al., 2014; Sundal et al., 2012), suggesting an important interplay of microglial and myelin abnormalities. Noteworthy, frontro-parietal alterations have been suggested in catatonia as well (Hirjak et al., 2020) and increased neuroinflammation and axonal swellings in the CC have been reported in aged $\mathrm{Cnp}^{+/}$ (Hagemeyer et al., 2012). Recent studies successfully attenuated axonopathy in mouse models of Charcot-Marie-Tooth (CMT) disease, a neuropathy modeled by myelin gene defects (Sereda et al., 1996; Sereda et al., 2006), upon depletion of macrophages via the CSF1-R inhibitor PLX3397 (Klein et al., 2015; Scherer, 2015). Usage of PLX3397 and PLX5622, targeting microglia more selectively (Dagher et al., 2015), further improved pathology and cognition in mouse models of AD and MS (Asai et al., 2015; Dagher et al., 2015; Groh et al., 2019; Nissen et al., 2018; Sosna et al., 2018).

Such therapeutic approaches seem promising, since studies suggest healthy genetic profiles (Huang et al., 2018), proper surveillance activities (Varvel et al., 2012) and even restored behavior (Dagher et al., 2015; Elmore et al., 2015; Elmore et al., 2014) upon replacement or repopulation. However, microglial subpopulations are known to have region- and contextspecific activities (Grabert et al., 2016; Xue et al., 2014), which can turn into pro- or antiinflammatory responses (Han et al., 2019). Thus, particularly the characterization of 


\section{Introduction}

autonomous subpopulations and their functions remains to be tackled. The thereby generated more profound knowledge on microglia as disease modifiers, could promote developing antiinflammatory therapeutic approaches to a variety of CNS disorders. Yet, the timepoint of application and impacts on other target cells or immunomodulatory microglia have to be considered in subsequent preclinical and clinical trials (Han et al., 2019; Nelson et al., 2017). 


\section{Introduction}

\section{Current perspectives in catatonia research}

As described above, catatonia was misperceived as a subtype of other medical conditions for a long period of time. The detachment from SSD in DSM-5 (2013), may enable urgently needed new diagnostic and experimental approaches, despite the failure to classify catatonia as an independent entity (Hirjak et al., 2020). Improved clinical rating scales, considering all symptomatic domains of catatonia, such as the Northoff Catatonia Rating Scale (NCRS) (Northoff, Koch, et al., 1999) may further promote reliability and accuracy of diagnosis (Oldham, 2019). Advanced technologies, such as targeted neuroimaging studies, are further essential to understanding catatonic symptoms on a functional and structural scope (Hirjak et al., 2020). Such advancements are crucial to improving clinical representation of a syndrome with a high comorbidity and heterogeneous symptomatology.

However, the lack of reliable animal models to date, continues to hamper research, particularly with regard to mechanistic insights. While not all features of catatonia can be sculpted or assessed in animal models, they constitute an important scientific tool in translational and therapeutic approaches. Previous studies from our group reported a catatonia-like phenotype in $\mathrm{Cnp}^{+/-}$and $\mathrm{Mbp} \mathrm{p}^{+/-}$mice upon aging (Hagemeyer et al., 2012; Poggi et al., 2016). Importantly, mice of both models exhibited signs of neuroinflammation, most prominently in frontal brain regions, which was also observed in human SSD carriers of a partial loss-of-function SNP in the CNP gene (Hagemeyer et al., 2012). Taking into account that dysregulated microglia as well as myelin abnormalities are increasingly recognized as disease modifiers (Frick et al., 2013; Nave \& Ehrenreich, 2014; Salter \& Stevens, 2017), the question emerges, whether neuroinflamamtion of subcortical white matter is causative to the observed phenotype or rather a mere consequence of other neuronal dysfunctions. Additionally, a potential improvement by depletion of abberant microglia remains to be evaluated, since such strategies are suggested as a promising therapeutic application in multiple CNS disorders (Han et al., 2019; Waisman et al., 2015). Noteworthy, inflammatory signs were observed in the frontal brain, suggesting a key function of inherent structures. Executive function, a higher-order cognitive ability, is known to be predominantly carried out by the frontal lobes (Alvarez et al., 2006; Otero et al., 2014; Stuss et al., 1984). It embraces a wide range of tasks including selective attention, working memory, cognitive flexibility and problem solving (Diamond, 2013). These functions are regulated by an interplay of multiple cortical structures (Carpenter et al., 2000), of which the PFC appears to be substantial (Chudasama, 2011; Yuan et al., 2014). Thus, the relevance of frontal lobe inflammation and executive dysfunction remain to be delineated and will help to clarify, whether 


\section{Introduction}

catatonia is driven by dysfunctional motor circuits or rather a frontal lobe disorder resulting in impaired volition and psychomotor behavior. 


\section{Introduction}

\section{Scope of the studies of the present work}

The clinical presentation of catatonia is heterogeneous and its widespread occurence throughout various CNS disorders is well known. Due to several shortcomings, including conceptual limitations and general misrepresentations, the etiology and underlying cellular pathomechanisms of the syndrome remain largely unclear to date. Importantly, research on catatonia has been hampered by these misperceptions and respectively inadequate or incomplete clinical rating scales. In addition, the scientific community is essentially lacking a targeted and reliable animal model, which in turn continues to retard mechanistic insights.

To this end, previous findings from our group reported a catatonia-like phenotype in C57Bl/6

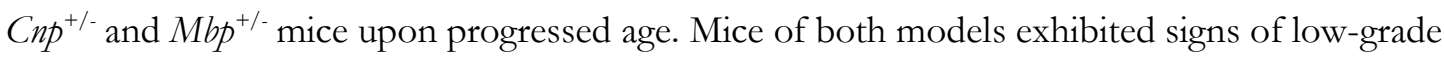
inflammation of the CNS, particularly observed in frontal brain structures. Interestingly, a comparable age-dependent catatonic phenotype was noted in human SSD carriers of a partial loss-of-function SNP in the CNP gene. Collectively, these findings suggest altered white matter integrity, possibly accompanied by abberant microglial activities, as key features of the observed phenotypes. However, critical question remain to be tackled, to assess whether and how neuroinflamation contributes to the catatonia-like phenotype. Moreover, the distinct roles of white matter integrity and the frontal brain need to be evaluated, aiming to enhance our understanding of the mechanisms resulting in the catatonia. Taking into account that dysregulated microglia as well as impaired white matter integrity are increasingly recognized as important disease modifiers, I aimed to address and characterize the relevance of these factors in the context of catatonia, a syndrome yet to be better understood and treated.

To address these questions we conducted experiments on both mice and men in my first project, with the former mainly focusing on $C n p^{-1-}$ mice. The aim of all respective experiments was to evaluate, whether neuroinflammtion of subortical white matter itself is indeed causative to previously reported findings. Accordingly, we investigated (i) the impact of age on exhibition of catatonic signs in our sample of schizophrenic subjects; (ii) the representation of the CNP lossof-function SNP rs2070106-AA in SSD subjects, in collaboration with Prof. Dr. Andreas Reif (iii) a potential impact of this SNP in healthy carriers on WMH, in collaboration with Prof. Dr. Hans Jörgen Grabe; (iv) the onset of catatonic signs in $C n p^{-1-}$ mice; (v) along with microgliatargeted therapeutical trials in an early intervention (prevention) and a late intervention (treatment) study; (v) immunohistochemical analysis of gliosis, neuroinflammation and degeneration within the $\mathrm{CC}$ and the cingulate cortex in the prevention and treatment study, 
respectively; (vi) neuroinflammation under microglia-targeted treatment and 5 weeks after cessation by magnetic resonance spectroscopy (MRS), in collaboration with Prof. Dr. Susann Boretius. A detailed description of the project along with respective findings are reported in Chapter 2 of this thesis.

Based on the findings from project I, we conducted further experiments on wildtype (WT) and $\mathrm{Cnp}^{-1-}$ mice, aiming to characterize microglial activities before and upon repopulation. We thereby recently reported the relevance of executive function in catatonia in mice and men (Garcia-Agudo et al., 2019). Executive function comprises a broad variety of cogntitive abilities (Diamond, 2013), which the frontal lobes are known to be critical to (Alvarez \& Emory, 2006; Otero \& Barker, 2014). To further delineate the role of neuroinflammation of the former in catatonia, I employed a second project in which we investigated and characterized mice lacking another important myelin gene, $P / p 1$, predominantly in the frontal brain. $P / p 1$-deficient mice have been reported to exhibit axonopathy and neuroinflammation (Griffiths et al., 1998; Lüders et al., 2019; Lüders et al., 2017) along with catatonia in adulthood, rendering them a suitable follow-up model of catatonia, in which we specifically targeted white matter integrity of the frontal brain.

In close collaboration with PD Dr. Hauke B. Werner and Prof. Klaus-Armin Nave Ph.D., we generated conditional knockout (cKO) by crossing mice with loxP site-flanked P/p1 alleles to mice expressing the Cre recombinase under the control of the Emx1 promoter promoter (Gorski et al., 2002). Subsequently, cKO mice were carefully characterized by (i) immunohistochemisty and (ii) neuropathological assessment, carried out by PD Dr. Hauke B. Werner and co-workers; (iii) extensive longitudinal behavioral phenotyping of mice of both genders, covering a wide range of behavioral domains such as motor and sensory function, cognition and specifically catatonia and executive function. A detailed description of the project along with respective findings are reported in Chapter 3 of this thesis. 
Chapter 2

\section{Project I}




\section{Project I}

\section{Microglia ablation alleviates myelin-associated catatonic signs in mice}

\section{Outline of the project}

Previous studies from our group reported an unexpected age-dependent behavioral phenotype reminiscent of the psychomotor syndrome of catatonia (Hagemeyer et al., 2012; Poggi et al., 2016). Mice heterozygous for the myelin genes Cnp and Mbp exhibited catatonic signs along with low-grade inflammation and microgliosis. The latter further showed significant reduction of myelination in the PFC upon aging, accompanied by reduction of both Mbp and Cnp as well as thinning of the CC (Poggi et al., 2016). Noteworthy, a similar age-dependent catatoniadepression phenotype was reported in SSD carriers of a partial loss-of-function SNP in the CNP gene resulting in increased inflammatory signs by DTI (Hagemeyer et al., 2012). In my doctoral thesis, I aimed to address the question whether neuroinflammation of subcortical white matter is causative to the observed phenotype in mice and men. Based on accumulating findings associating aberrant microglial functions with neuropsychiatric disorders (Frick et al., 2013) and implications of therapeutic benefits by microglial depletion and subsequent repopulation (Han et al., 2018; Waisman et al., 2015), I further investigated the impact of the CSF1-R inhibitor PLX5622 (Dagher et al., 2015) on the onset and progression of the phenotype by behavioral, immunohistochemical and neuroimaging measures of distinct target sites.

\section{(I) Assessment of catatonic signs in subjects suffering from SSD}

A total of 1095 subjects from the GRAS schizophrenia sample were evaluated for potential catatonic signs and their severity by application of the Cambridge Neurological Inventory (CNI) catatonia score (Chen et al., 1995). These analyses were carefully carried out by our co-worker Dr. Martin Begemann and revealed an age-dependent increase in the CNI catatonia score in SSD subjects. Subsequently, Marina Mitjans, Ph.D., conducted a genome-wide association study (GWAS) on all subjects to investigate the representation of carriers of the CNP SNP rs2070106 within groups exhibiting no CNI catatonia score up to subjects with a score of 2 or higher. Comparison of extreme groups revealed approximately $18 \%$ of the latter to be carriers of the SNP. This finding was further confirmed in another independent sample of SSD patients $(\mathrm{N}=288)$ by Prof. Dr. Andreas Reif and co-workers. 


\section{Project I}

\section{(II) Impact of the SNP rs2070106 on white matter hyperintensities}

To tackle this question, we carried out another GWAS on the baseline cohort of SHIPTREND-0 (N=552; Völzke et al., 2011) in close collaboration with Prof. Dr. Hans Jörgen Grabe and co-workers. Healthy subjects with avaialbale magnetic resonance imaging (MRI) and SNP information were included into the study and revealed an age-dependent increase in white matter hyperintensities (WMH), which was significantly exceeded by carriers of the SNP. Regional anaylsis of CNS areas most affected suggested particularly frontotemporal regions as such.

\section{(III) Onset and prevention of catatonic signs in $\mathrm{Cnp}^{-/-}$mice}

Since we previously observed a catatonia-depression phenotype in $\mathrm{Cn}^{+/ \text {- }}$ mice upon progressed age (Hagemeyer et al., 2012), we initially addressed the question whether homozygous KO would facilitate the phenotype. Analysis of the Bar test data revealed catatonic-signs in $\mathrm{Cnp}^{-1}$ mice as early as 8 weeks age. Subsequently, WT and $C n p^{-1-}$ mice were fed with PLX5622 or control chow for 5 weeks and tested in the Bar test at 8 weeks age. Indeed, microglia-targeted treatment with PLX5622 alleviated catatonia in $C n p^{-1-}$ mice, while it did not affect WT behavior. To address the question whether depletion of microglia would have a sustained impact on neuropathology and inflammation, observed in untreated $C_{n} p^{-1}$ mice, we conducted immunohistochemical labeling of ionized calcium-binding adapter molecule 1 (IBA1), amyloid precursor protein (APP) and glial fibrillary acidic protein (GFAP) in the CC of cross-sectioned brain slices 4 weeks after cessation of treatment. Surprisingly, treatment with PLX5622 sustainably reduced the numer of Iba1- and APP-positive (IBA1 ${ }^{+}$and $\mathrm{APP}^{+}$, respectively) cells in $\mathrm{Cn}^{-/}$mice, while the number of $\mathrm{GFAP}^{+}$cells remained elevated in treated and untreated $\mathrm{Cnp}$ /- mice. These findings strongly suggested a prevention of the catatonia-like phenotype in $\mathrm{Cnp}^{-1}$ mice along with diminished signs of neuropathology in white matter tracts. The latter was confirmed by MRS in close collaboration with Prof. Dr. Susann Boretius and co-workers, since myoinositol, an appreciated marker of glial activation and neuroinflammation (Chang et al., 2013; Poggi et al., 2016; Ross et al., 1997), was reduced in the CC and cortex of PLX5622treated $\mathrm{Cnp}^{- \text {- }}$ mice, even 5 weeks after cessation of treatment.

\section{(IV) Treatment of catatonic signs upon disease progression in $C_{n p^{-/-}}$mice}

Since we efficiently intercepted the catatonic phenotype in the above-mentioned studies, we next aimed to investigate, whether microglial depletion via PLX5622 would also benefit the 
phenotype at a more progressed state. To this end, WT and $C n p^{-/}$mice were treated with PLX5622 and control chow for a total of 8 weeks starting at the age of 27 weeks. Analysis of the Bar test after 5 and 8 weeks of treatment successfully attenuated catatonic signs in $\mathrm{Cnp}^{\%}$ mice, while treatment did not affect WT behavior. Analysis of neuropathology under 8 weeks of treatment further revealed significant reduction of microgliosis $\left(\mathrm{IBA}^{+}\right)$and phagocytic microglial activities $\left(\mathrm{CD}^{+} 8^{+}\right)$, axonal spheroids $\left(\mathrm{APP}^{+}\right)$, astrogliosis $\left(\mathrm{GFAP}^{+}\right)$and infiltrating $\mathrm{T}$ lymphocytes $\left(\mathrm{CD}^{+}\right)$, all of which were drastically increased in the $\mathrm{CC}$ of untreated $\mathrm{Cnp} \mathrm{p}^{-/}$mice. Morever, $\mathrm{KO}$ mice exhibited an expected increase in OPCs in the CC, which was counteracted by PLX5622, irrespective of genotypes. Interestingly, IBA $1^{+}$cells were increased in the cingulate cortex of $\mathrm{Cn}^{-1-}$ mice and successfully reduced by PLX5622 treatment, suggesting expansion of inflammatory processes into the frontal gray matter in $\mathrm{Cnp}^{-1}$ mice.

\section{(V) Catatonic signs in myelin mutant mice and other mouse models of CNS disease}

In conclusion, we investigated whether the observed catatonic signs are restricted to mice with altered white matter integrity due to myelin gene deficiencies and neuroinflammatory processes., I conducted the Bar test with a battery of mutant mice including $C n p^{+/}, M b p^{+/}, P h p^{\text {mullly }}$. The former two exhibited catatonic signs upon progressed age, as reported previously (Hagemeyer et al., 2012; Poggi et al., 2016) while the later showed catatonic signs at an earlier time point during adulthood. As a proof-of-principle experimental approach, I tested various other mouse models with deficiencies of the autophagy and beclin 1 regulator gene (Ambra ${ }^{+/}$; Dere et al.,

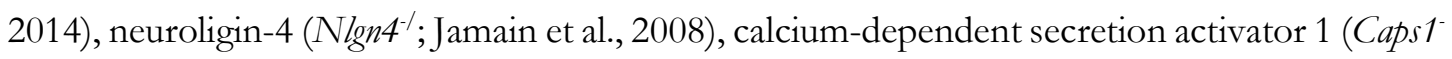
'; Jockusch et al., 2007), postsynaptic density protein-93 and -95 (PSD93\% al., 2018) and apolipoprotein-E (ApoE $E^{-/}$; Hammer et al., 2014), all of which did not exhibit catatonic signs at any time. These findings strongly suggested a critical role of altered white matter integrity and neuroinflammatory processes to be causative to the observed phenotype, at least in our mouse models of catatonia. 


\section{Project I}

\section{Peer-reviewed publication}

Janova, H.*, Arinrad, S.*, Balmuth, E.*, Mitjans, M., Hertel, J., Habes, M., Bittner, R.A., Pan, H., Goebbels, S., Begemann, M., Gerwig, U.C., Langner, S., Werner, H.B., Kittel-Schneider, S., Homuth, G., Davatzikos, C., Völzke, H., West, B.L., Reif, A., Grabe, H.J., Boretius, S., Ehrenreich, H. and Nave, K.A. (2018). Microglia ablation alleviates myelin-associated catatonic signs in mice. Journal of Clinical Investigation, 128(2):734-745.

* Shared first authorship.

Personal contributions: Under supervision of Prof. Dr. Dr. Hannelore Ehrenreich, I was particularly responsible for all behavioral mouse studies conducted in the process of this publication. Hence, I conducted the Bar test in animals of all cohorts including the initial comparison of WT versus $C n p^{+/}$and $C n p^{-1}$ mice (Figure 2A) as well as mice of the prevention and the treatment studies (Figures 2B-D, 4A-B). All data acquired with the Bar test were analyzed and prepared by myself (Figures 2A, 2D, 4B, 5G). Moreover, I performed and monitored treatment of all mice from the prevention, the treatment and the MRS studies with control diet or PLX5622 (Figures 2D, 3B-G, 4B). The latter experiments were carried out in collaboration with Prof. Dr. Susann Boretius. I further conducted enzymatic immunohistochemical labeling and quantification of $\mathrm{APP}^{+}$swellings within the CC of mice from the prevention and treatment studies (Figures 2F, 5A). Together with Dr. Hana Janova, I carried out statistical analyses of all data acquired by studies in mice. In conclusion, I was also actively involved in the assembly of figures and display items as well as preparation of the manuscript itself. Specifically, I contributed by writing the material and methods sections involving mouse maintenance and behavioral experiments as well as description of applied statistical analyses within the caption of figures 2 to 5 . 


\title{
Microglia ablation alleviates myelin-associated catatonic signs in mice
}

\author{
Hana Janova, ${ }^{1,2}$ Sahab Arinrad, ${ }^{1}$ Evan Balmuth, ${ }^{1}$ Marina Mitjans, ${ }^{1,2}$ Johannes Hertel, ${ }^{3}$ Mohamad Habes, ${ }^{3,4}$ Robert A. Bittner, ${ }^{5}$ \\ Hong Pan, ${ }^{1}$ Sandra Goebbels, ${ }^{2,6}$ Martin Begemann, ${ }^{1,2,7}$ Ulrike C. Gerwig, ${ }^{6}$ Sönke Langner, ${ }^{8}$ Hauke B. Werner, ${ }^{6}$ \\ Sarah Kittel-Schneider, ${ }^{5}$ Ceorg Homuth, ${ }^{9}$ Christos Davatzikos, ${ }^{4}$ Henry Völzke, ${ }^{10}$ Brian L. West, ${ }^{11}$ Andreas Reif, ${ }^{5}$ \\ Hans Jörgen Grabe, ${ }^{3}$ Susann Boretius, ${ }^{2,12}$ Hannelore Ehrenreich, ${ }^{1,2}$ and Klaus-Armin Nave ${ }^{2,6}$ \\ 'Clinical Neuroscience, Max Planck Institute of Experimental Medicine, Cöttingen, Germany. ${ }^{2} \mathrm{DFG}$ Research Center for Nanoscale Microscopy and Molecular Physiology of the Brain (CNMPB), Cöttingen, \\ Germany. ${ }^{3}$ Department of Psychiatry and Psychotherapy, University Medicine, and German Center for Neurodegenerative Diseases (DZNE), Greifswald, Germany. ${ }^{4}$ Center for Biomedical Image Computing \\ and Analytics, University of Pennsylvania, Philadelphia, Pennsylvania, USA. 5Department of Psychiatry, Psychosomatic Medicine and Psychotherapy, University Hospital, Goethe University, Frankfurt, \\ Germany. ${ }^{6}$ Department of Neurogenetics, Max Planck Institute of Experimental Medicine, Göttingen, Germany. 'Department of Psychiatry and Psychotherapy, University Medical Center Cöttingen (UMG), \\ Georg-August-University, Göttingen, Germany. ${ }^{8}$ Institute of Diagnostic Radiology and Neuroradiology, ${ }^{9}$ Interfaculty Institute for Genetics and Functional Genomics, and ${ }^{10}$ Institute for Community Medicine, \\ University Medicine Greifswald, Greifswald, Germany. ${ }^{11 T r a n s l a t i o n a l ~ P h a r m a c o l o g y, ~ P l e x x i k o n ~ I n c ., ~ B e r k e l e y, ~ C a l i f o r n i a, ~ U S A . ~}{ }^{2}$ Functional Imaging Laboratory, Leibniz Institute for Primate Research, \\ Göttingen, Germany.
}

\begin{abstract}
The underlying cellular mechanisms of catatonia, an executive "psychomotor" syndrome that is observed across neuropsychiatric diseases, have remained obscure. In humans and mice, reduced expression of the structural myelin protein CNP is associated with catatonic signs in an age-dependent manner, pointing to the involvement of myelin-producing oligodendrocytes. Here, we showed that the underlying cause of catatonic signs is the low-grade inflammation of white matter tracts, which marks a final common pathway in Cnp-deficient and other mutant mice with minor myelin abnormalities. The inhibitor of CSF1 receptor kinase signaling, PLX5622, depleted microglia and alleviated the catatonic symptoms of Cnp mutants. Thus, microglia and low-grade inflammation of myelinated tracts emerged as the trigger of a previously unexplained mental condition. We observed a very high $(25 \%)$ prevalence of individuals with catatonic signs in a deeply phenotyped schizophrenia sample $(n=1095)$. Additionally, we found the loss-of-function allele of a myelin-specific gene (CNP rs2070106AA) associated with catatonia in 2 independent schizophrenia cohorts and also associated with white matter hyperintensities in a general population sample. Since the catatonic syndrome is likely a surrogate marker for other executive function defects, we suggest that microglia-directed therapies may be considered in psychiatric disorders associated with myelin abnormalities.
\end{abstract}

\section{Introduction}

White matter tracts in the CNS largely comprise long axons, associated glial cells, and the ensheathment of axons with myelin. While the role of myelin for axonal conduction and normal motor-sensory function is well known (1), the contribution of white matter integrity to cortical networks and higher cognition is just emerging. Moreover, myelin defects are increasingly linked to mental disease, but mechanistic insight is still lacking and the relationship between cause and consequence difficult to establish in humans $(2,3)$.

Catatonia is among the most mysterious and as yet poorly understood neuropsychiatric phenotypes. Appearing as a "psychomotor syndrome," it reflects temporary disruption of executive control in the absence of any "classical" motor dysfunction. Cata-

Related Commentary: https://doi.org/10.1172/JCI98761

Authorship note: $\mathrm{H}$. Janova, S. Arinrad, and E. Balmuth contributed equally to this work. H. Ehrenreich and K.A. Nave contributed equally to this work. Conflict of interest: Brian West is an employee of Plexxikon Inc. Submitted: August 22, 2017; Accepted: November 7, 2017. Reference information: / Clin Invest. https://doi.org/10.1172/JCI97032. tonia is typically characterized by a fluctuating course with episodic exacerbations and has historically been associated with schizophrenia, for which it is classified as a positive symptom. Catatonia is, however, also observed in mood- and substance-induced psychotic disorders, malignant neuroleptic syndrome, most encephalitides, and even general medical conditions $(4,5)$. Reports on brain areas involved in catatonia are scarce. Available data point to frontal lobe regions, such as the pronounced catatonia in a case of butterfly glioma of the frontal corpus callosum (6) or frontal activation in akinetic catatonic patients detected by functional MRI (7).

The full-blown clinical picture of catatonia is dominated by immobility, catalepsy, or stupor, sometimes suddenly switching from "frozen posturing" to excessive motor activity ("movement storm"). Milder forms are more common, even though frequently missed in the diagnostic process, and addressed as catatonic signs (8). In schizophrenia, prevalence is estimated at $0.2 \%-3.0 \%$ (5). Treatment with benzodiazepines or electroconvulsive therapy is nonspecific and not always effective $(4,5)$.

Similarly to what occurs in humans, catatonia appears in mice as a state of transient immobility in which mice persist in an externally imposed abnormal posture. However, in animals, catatonia has previously been reported only upon induction by body pinch 


\section{Project I}

Downloaded from http://www.jci.org on December 19, 2017. https://doi.org/10.1172/JCI97032

RESEARCH ARTICLE

A

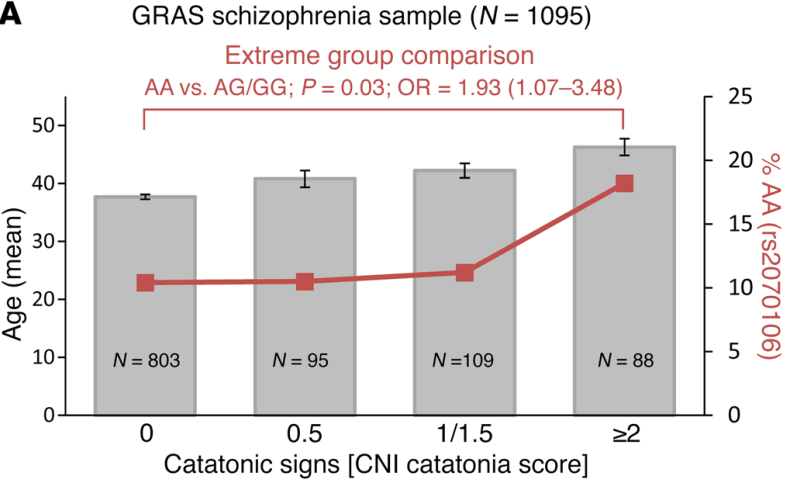

B Würzburg schizophrenia replication sample $(N=288)$ Distribution of rs2070106 genotype
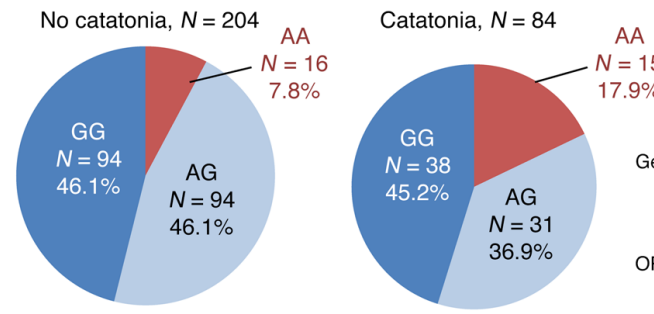

AA

$7.9 \%$

Genotypic comparison

$P=0.035$

$A A$ vs. $A G / G G$

$P=0.015$

$\mathrm{OR}=2.55(1.19-5.44$

\section{General population (SHIP-TREND-0; $N=552$ ) White matter hyperintensities (WMH)}
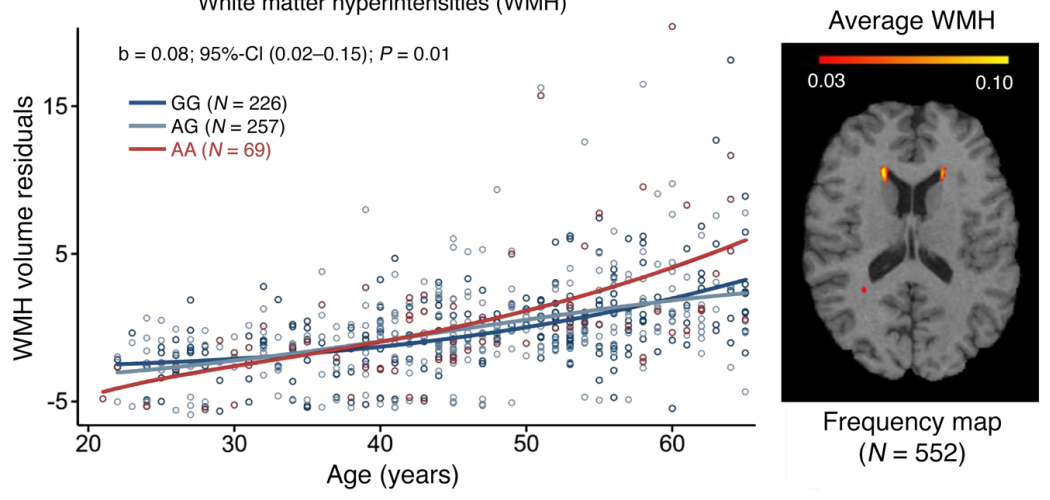

The Journal of Clinical Investigation
Figure 1. Age-dependent association of the lossof-function genotype CNP rs2070106-AA with catatonia in 2 independent schizophrenia cohorts and with WMH in a general population sample. (A) Bars show mean age of schizophrenic subjects (GRAS), sorted by severity of catatonic signs. Red line denotes percentage of risk for genotype carriers (rs2070106-AA) within each severity group. Note that severity of catatonic signs increases with age. Two-sided values for Kruskal-Wallis $(P=$ $\left.7.6 \times 10^{-9}\right)$ and Jonckheere-Terpstra $\left(P=1.3 \times 10^{-9}\right)$. Mean \pm SEM. Also note that CNP rs2070106-AA carriers are most frequent (18.2\%) among individuals with highest expression of catatonic signs compared with noncatatonic subjects (10.4\%). Two-sided $P$ value for Mann-Whitney $U$ test for extreme-group comparison given in the figure. (B) Distribution of CNP rs2070106 genotype in the Würzburg replication sample of schizophrenia patients based on dichotomous catatonia classification. The AA genotype is significantly more prevalent in patients with (17.9\%) than without catatonia (7.8\%). Two-sided $P$ values from $\chi^{2}$ test given. (C) Left panel: interaction effect between age and genotype in SHIP-TREND-O sample on overall WMH volume (minimum $10 \mathrm{~mm}^{3}$ per single WMH cluster). Shown are WMH volume residuals after correcting for intracranial volume, age (nonlinear), and gender. Genotype and age-genotype interaction term contributed $1.1 \%$ of variance to overall WMH volumes. Right panel: frequency map averaging all subjects of the general population (SHIP-TREND-0; $n=552$ ), analyzed here. Data highlight WMH appearance predominantly in frontal regions. or drug exposure $(9,10)$. More recently, we detected catatonia in aging mice heterozygous for either Cnp or Mbp (also known as shiverer), both encoding structural proteins of the myelin sheath, 2'-3'-cyclic nucleotide 3'-phosphodiesterase (Cnp) and myelin basic protein (Mbp). Interestingly, in aging $M b p$ heterozygotes, Cnp expression in the forebrain is also reduced by $50 \%(11,12)$. Correspondingly, individuals homozygous for the A allele of the SNP rs2070106 in human CNP show reduced mRNA expression (13) and association with catatonia (11). Therefore, mutant oligodendrocytes yield a predisposition to catatonia, which is not explained by any paucity of myelin; however, the responsible mechanisms have remained obscure.

The neuropathology of Cnp and Mbp heterozygous mice starts late in life and is surprisingly mild, but in either mutant accompanied by an increased number of microglial cells $(11,12,14)$. We therefore hypothesized that neuroinflammation of subcortical white matter, possibly spreading into the prefrontal cortex, could be the cause of catatonic signs. Such a mechanism would be in line with the emerging role of microglia for behavioral phenotypes (15-18).

Here, we demonstrate an unexpectedly high, age-associated prevalence of catatonic signs in more than $25 \%$ of deeply phenotyped schizophrenic subjects. In 2 independent schizophrenia cohorts, we found severe catatonic signs associated with the CNP partial loss-of-function genotype rs2070106-AA. Moreover, we show by MRI that CNP rs2070106-AA carriers in the general population are more likely than $\mathrm{G}$ carriers (GG or AG) to display frontotemporal white matter hyperintensities $(\mathrm{WMH})$ on T2-weighted images as proposed subclinical signs of vascular changes, neuroinflammation, and demyelination (19-21).

To provide proof-of-principle for microgliosis as the key disease mechanism, we studied $\mathrm{Cnp}^{-/}$(null) mutant mice. Surprisingly, they developed neuroinflammation with catatonic signs by the early age of 8 weeks. Indeed, we show causality by depletion of microglia with the colony-stimulating factor 1 receptor (CSF1R) 


\section{Project I}

Downloaded from http://www.jci.org on December 19, 2017. https://doi.org/10.1172/JCI97032

\section{Table 1. ROI analyses of WMH in analogous regressions showing that frontotemporal regions including deep structures contribute most to the overall increase of WMH in AA carriers versus subjects with AG/GG genotype}

\begin{tabular}{|c|c|c|c|}
\hline Brain region & $\begin{array}{c}\text { Age-genotype interaction }{ }^{\mathrm{A}} \\
(95 \% \mathrm{Cl})\end{array}$ & $P$ value & $\begin{array}{c}\text { Variance contribution } \\
\left(\Delta \text { adj. } R^{2}\right)\end{array}$ \\
\hline Frontal & $0.06(0.01 ; 0.10)$ & 0.013 & $0.68 \%$ \\
\hline Deep structures ${ }^{\mathrm{B}}$ & $0.02(0.00 ; 0.03)$ & 0.048 & $0.36 \%$ \\
\hline Parietal & $0.05(-0.00 ; 0.09)$ & 0.054 & $0.71 \%$ \\
\hline Temporal & $0.05(0.00 ; 0.10)$ & 0.032 & $1.17 \%$ \\
\hline Occipital & $0.02(-0.02 ; 0.05)$ & 0.333 & $0.13 \%$ \\
\hline
\end{tabular}

${ }^{A}$ Regression weight. ${ }^{B}$ Deep structures include anterior limb of internal capsule, fornix, and posterior limb of internal capsule including cerebral peduncle. $\Delta$ adj. $\mathrm{R}^{2}$ calculated by comparing the whole model with the model devoid of the genotype and the agegenotype interaction term. Analyses are adjusted for intracranial volume, age (nonlinear), and gender. All $P$ values are 2 sided. Bold numbers indicate $P<0.05$.

inhibitor PLX5622 $(22,23)$, which blocks a critical microglial survival pathway. The so-caused ablation of microglia prevents catatonia onset in young mutants and ameliorates existing catatonia in adult $\mathrm{Cnp}^{-/-}$mice. These findings shed light on the nature of catatonia and suggest that this striking neuropsychiatric syndrome and possibly related executive function deficits - may be preventable as well as treatable.

\section{Results}

Catatonic signs are highly prevalent in schizophrenia and associated with the CNP partial loss-of-function genotype rs2070106-AA in independent samples. Within the Göttingen Research Association for Schizophrenia (GRAS) population of deeply phenotyped schizophrenic subjects, $26.7 \%$ exhibited signs of catatonia. The severity of catatonic signs clearly increases with age (Figure 1A). The percentage of CNP loss-of-function SNP rs2070106-AA carriers among individuals with the highest expression $(\geq 2)$ of catatonic signs is greater compared with that in noncatatonic subjects (18.2\% versus $10.4 \% ; P=0.03 ; \mathrm{OR}=1.93$; Figure $1 \mathrm{~A})$. This association between catatonia and rs2070106-AA is replicable in an independent sample of schizophrenic individuals (17.9\% versus $7.8 \% ; P=0.015 ; \mathrm{OR}=2.55$; Figure $1 \mathrm{~B})$, categorically classified for catatonia according to Leonhard (24).

CNP rs2070106-AA carriers in the general population display increased age-dependent WMH in frontal and temporal brain areas. We wondered whether the CNP loss-of-function rs2070106-AA would reveal any measurable effects on suggested MRI indicators of neuroinflammation and white matter alterations. Employing a subsample of the baseline cohort of Study of Health in Pomerania (SHIP-TREND-0), namely general population subjects with MRI scans available, we quantified WMH. AA carriers showed age-dependent higher WMH volume residuals as compared with GG and AG carriers, most prominently in frontotemporal brain regions and deep brain structures, with all analyses adjusted for total intracranial volume, age (nonlinear), and gender (Figure 1C and Table 1). Control covariance analyses, stepwise including further (potentially interfering) covariates alone or together, namely education, waist circumference, serum triglycerides, and smoking, did not appreciably alter the results. Importantly, peripheral inflammation markers, namely C-reactive protein serum levels (natural logarithm) and white blood cell count were tested as covariates, but also did not substantially change the significance level $(P=0.013$ and $P=0.014$, respectively).

Catatonic signs in Cnp-null mutant mice start at around 8 weeks of age and are prevented by the CSF1R inhibitor PLX5622. To show proof-of-principle for the causal relationship of inflammation and catatonic signs in a construct-valid experimental model, we turned to $\mathrm{Cnp}^{-/-}$mutant mice. As early as the age of 8 weeks, $\mathrm{Cnp}^{-/-}$mice developed catatonic signs (Figure 2, A and B). For illustration, Supplemental Video 1 (supplemental material available online with this article; https:// doi.org/10.1172/JCI97032DS1) demonstrates a striking example of catatonia in a young $\mathrm{Cnp}^{-/-}$mutant, tested on the bar, followed by normal-appearing motor performance. This exemplifies the transiently obvious executive dysfunction in the absence of an underlying motor disturbance. Supplemental Video 2 shows excerpts of undisturbed home-cage observation in an enriched environment of another $\mathrm{Cnp} \mathrm{p}^{-/}$mutant with normal motor performance and phases of spontaneous catatonia-like posturing (note the "manneristic" stretching of hind limbs when on the bar).

Catatonic signs were prevented by a 5-week oral application of the CSF1R inhibitor PLX5622 via food pellets, starting at 3 weeks of age, immediately after weaning (Figure 2, C and D), consistent with a nearly complete depletion of microglia as reported earlier $(22,23)$ and reproduced by pilot experiments in preparation of the present study (8-week-old WT mice, treated for 5 or 8 weeks with PLX5622 versus untreated: 1-2 versus 260 ionized calcium binding adaptor molecule- 1 positive [ $\left.\mathrm{Iba1}^{+}\right]$cells $/ \mathrm{mm}^{2}$ corpus callosum area, as delineated in Figure 2E). Intriguingly, immunohistochemical (IHC) analysis of the corpus callosum of these mice after 4 weeks of drug recovery showed still lower numbers of Iba $1^{+}$ cells and amyloid precursor protein-positive $\left(\mathrm{APP}^{+}\right)$swellings compared with nontreated $\mathrm{Cnp} \mathrm{p}^{-/-}$mice, suggesting that PLX5622 treatment has a persistent antiinflammatory benefit, since it is known that microglia recover from inhibition within only 1 week $(22,23)$. The slightly enhanced glial fibrillary acidic protein-positive $\left(\mathrm{GFAP}^{+}\right)$area (Figure 2, $\mathrm{E}$ and $\mathrm{F}$ ) as well as the mildly extended $\mathrm{CD}^{\circ} 8^{+}$(macrosialin) area $(0.8 \% \pm 0.04 \%$ in WT versus $6.8 \% \pm$ $0.7 \%$ in $\left.\mathrm{Cnp}^{-1-} ; \mathrm{P}<0.014\right)$ in young mutants is not reduced after PLX5622 treatment.

Magnetic resonance spectroscopy signs of white and gray matter inflammation in $\mathrm{Cnp}^{-/}$mice and their prevention by CSF1R inhibition. An independent cohort of WT and $\mathrm{Cnp}^{-/-}$mice underwent a follow-up magnetic resonance spectroscopy (MRS) study at 8 and 13 weeks of age (design shown in Figure 2C), focusing on regions of interest (ROI) in corpus callosum (white matter) and cortex (gray matter) (Figure 3A). The first MRS in 8-week-old mice was performed after 5 weeks of control versus PLX5622 diet (starting at age 3 weeks, as in the prevention study above), and the second MRS was performed in the same mice at the age of 13 weeks, i.e., after 5 weeks of PLX5622 food cessation/microglia repopulation. Brain myoinositol is seen as a global marker of glial activation including microglia that strongly correlates with neuroinflam- 


\section{Project I}

Downloaded from http://www.jci.org on December 19, 2017. https://doi.org/10.1172/JCI97032

RESEARCH ARTICLE

The Journal of Clinical Investigation

A Catatonic signs in $\mathrm{Cnp}$ mutant mice (age 8 weeks)

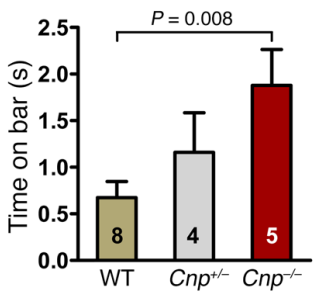

B

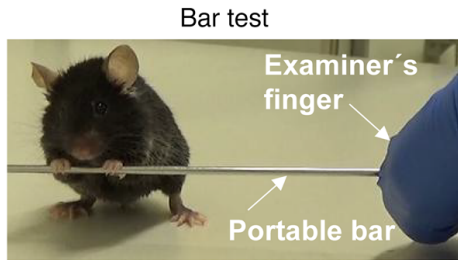

C Prevention study design: CSF1R inhibition

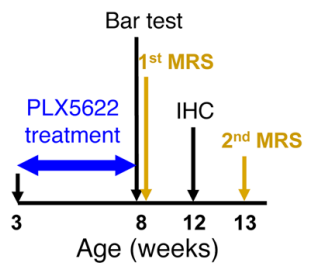

D Catatonic signs after 5 weeks PLX5622

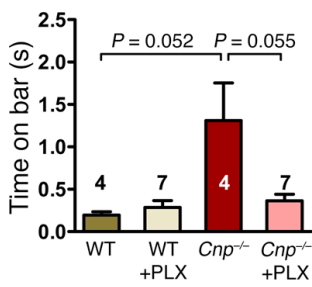

$\mathbf{E}$ Areas of $\mathrm{IHC}$ quantifications in $\mathrm{CC}$ and $\mathrm{Cg}$ cortex

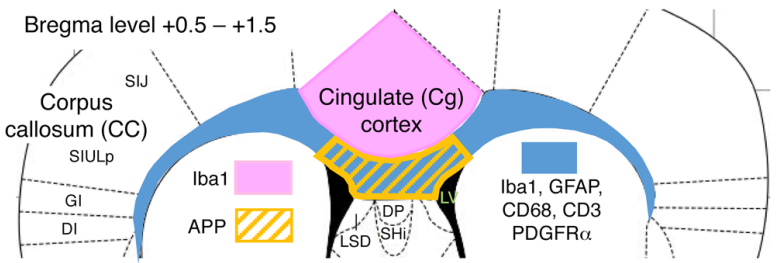

$\mathbf{F}$

IHC: Corpus callosum after 5 weeks of PLX5622 + 4 weeks of microglial repopulation
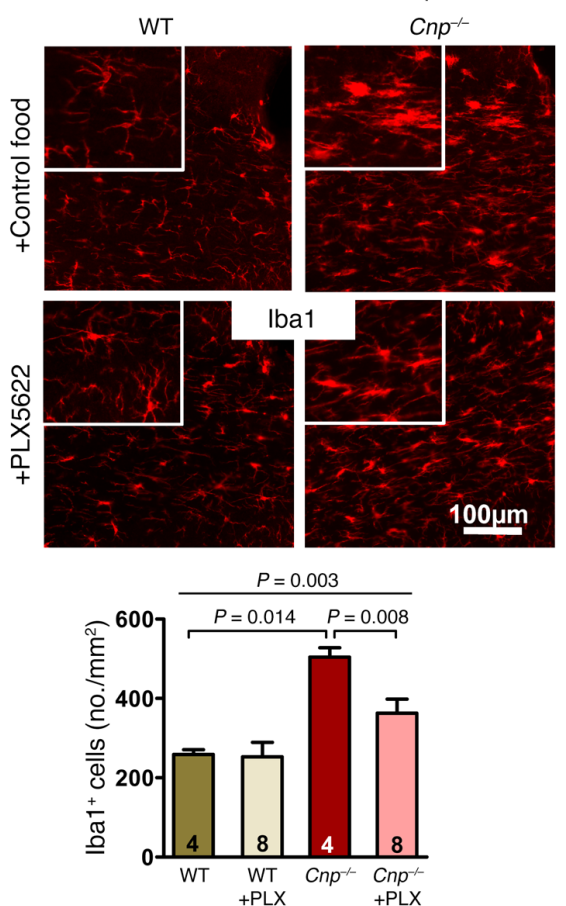

WT

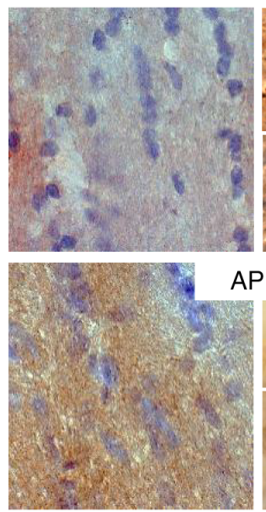

$\mathrm{Cnp}^{-1-}$
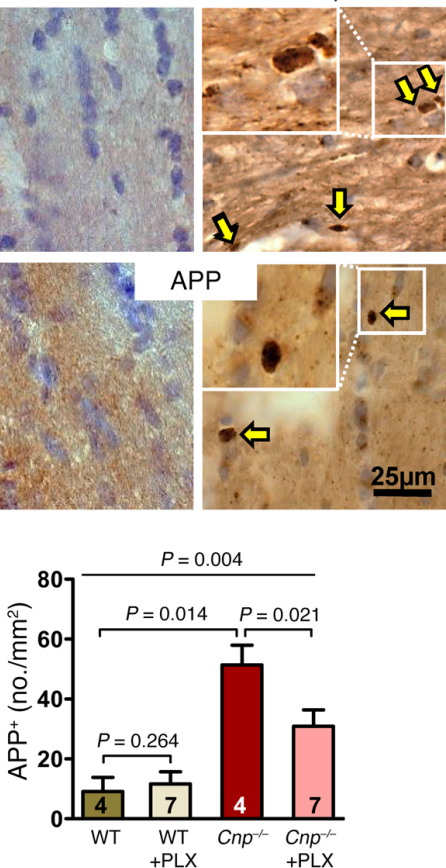

WT
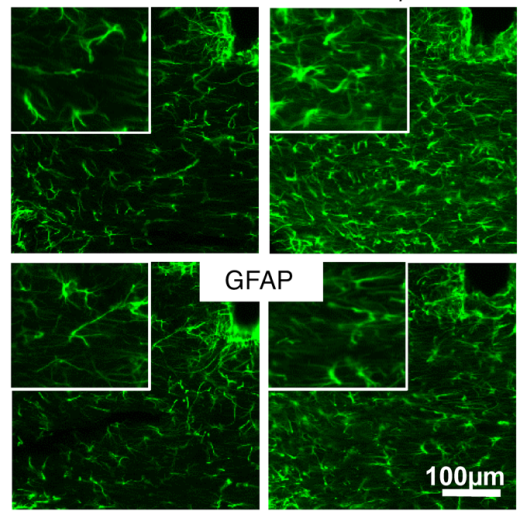

AP

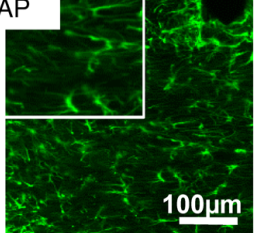

Figure 2. Early catatonia and white matter inflammation in Cnp mutant mice and their prevention by CSF1R inhibition. (A) Catatonic signs measured by the bar test in WT, $\mathrm{Cnp}^{+/-}$, and $\mathrm{Cnp}^{-/-}$mice at the age of 8 weeks (Kruskal-Wallis, $\left.P=0.034\right)$. (B) Image illustrating a mouse with typical catatonic posture during the bar test. (C) Schematic overview of the prevention study design, including PLX5622 (versus regular food) feeding phase (blue arrow) and time points of testing/analyses. Black arrows: bar test (results in Figure 2D) and IHC (results in Figure 2F); yellow arrows: MRS measurements (results in Figure 3). (D) Catatonic signs in WT and Cnp ${ }^{-/-}$mice (age 8 weeks) after 5-week PLX5622 or control food diet (Kruskal-Wallis, $P=0.165$ ). (E) Schematic overview illustrating corpus callosum and neighboring cingulate cortex areas for IHC quantifications: defined ROI for quantifying APP+ swellings, Iba1 ${ }^{+}$and CD ${ }^{+}$cells as well as GFAP ${ }^{+}$and $\mathrm{CD}_{8} 8^{+}$areas (densitometric analysis) shown by the yellow striped field and the blue area, respectively; cingulate area (Cg1/Cg2) for

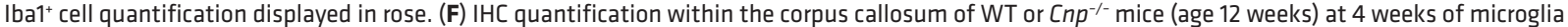
repopulation after 5 weeks of PLX5622 or control diet, as shown in C and E. Upper panels show representative images, including higher-magnification inserts, of the quantifications shown underneath: Iba1 ${ }^{+}$cells (no. $/ \mathrm{mm}^{2} ; 1$ section/brain), APP ${ }^{+}$swellings (yellow arrows indicating APP ${ }^{+}$spheroids; no./mm; 3 sections/brain), and densitometric analysis of GFAP+ area (\%; 1 section/brain). Original magnification (insets), $\times 2$ (Iba1, GFAP); $\times 4$ (APP). All data in A, $\mathbf{D}$, and $\mathbf{F}$ were individually tested for Gaussian distribution using the Kolmogorov-Smirnov test. Nonparametric Kruskal-Wallis test was performed for $\mathbf{A}$, D and $\mathbf{F}$ for multiple group comparisons, followed by post hoc 1-tailed Mann-Whitney $U$ test. All data are shown as mean \pm SEM; $n$ indicated within bars. 


\section{Project I}

Downloaded from http://www.jci.org on December 19, 2017. https://doi.org/10.1172/JCI97032

The Journal of Clinical Investigation

RESEARCH ARTICLE

A

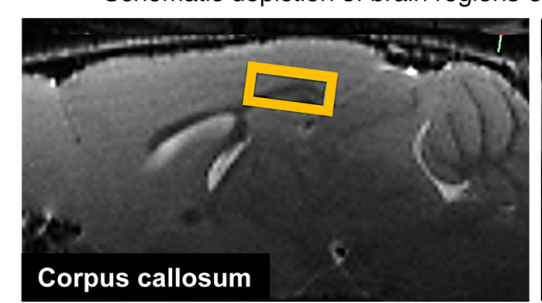

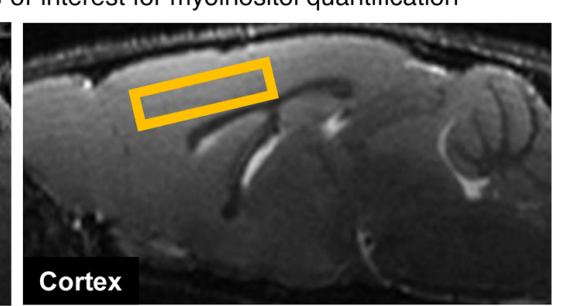

Corpus callosum
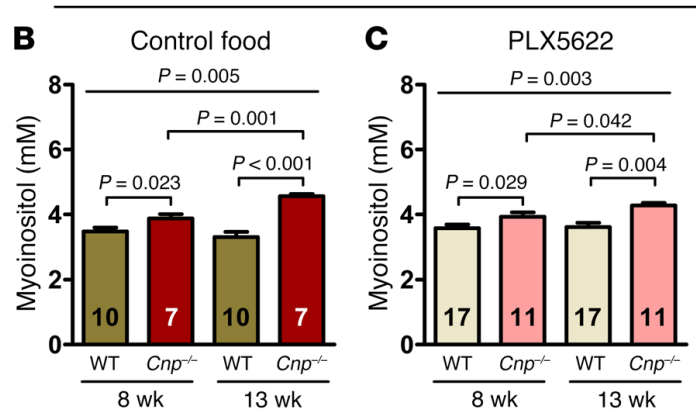

Cortex
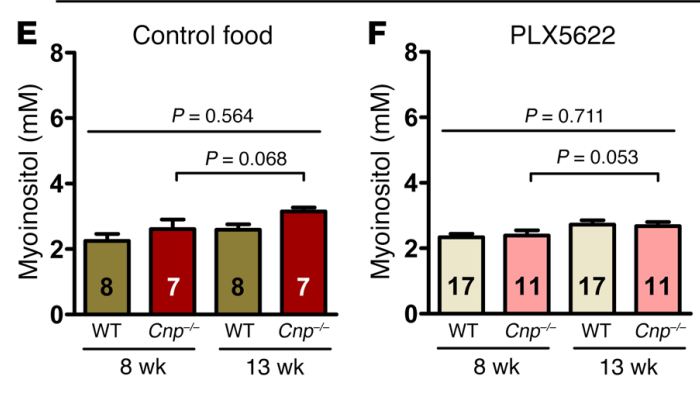

D

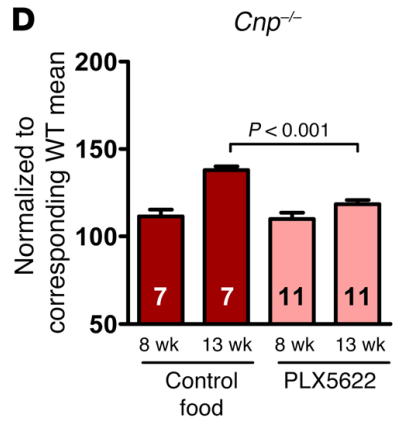

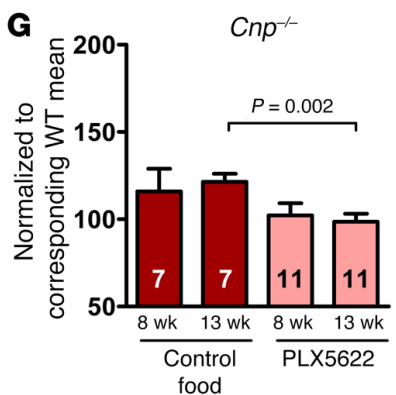

Figure 3. MRS signs of white matter (corpus callosum) and gray matter (cortex) inflammation (myoinositol) in Cnp $^{-/-}$mice and prevention by CSF1R inhibition.

(A) Representative sagittal MR images illustrating corpus callosum and cortex ROI for analysis of myoinositol levels (yellow squares). (B-D) Corpus callosum: follow-up MRS for quantification of myoinositol in WT and $\mathrm{Cnp}^{-/-}$mice at 8 and 13 weeks of age (experimental design shown in Figure 2C). Statistical comparison of the first MRS in 8-week-old mice, after 5 weeks of control (B) versus PLX5622 (C) diet (starting at age 3 weeks) and the second MRS in these same mice at the age of 13 weeks, after 5 weeks of regular food (repopulation after PLX5622). (D) Note the return to nearly WT level in PLX5622-treated Cnp ${ }^{-/-}$mice. (E-G) Cortex: same design as for corpus callosum. (E) Inflammatory phenotype of $\mathrm{Cnp} /$ mice less pronounced. ( $\mathbf{F}$ and $\mathbf{G}$ ) Effect of PLX5622 less prominent. All data in B-G were individually tested for Gaussian distribution using the Kolmogorov-Smirnov test. Two-way ANOVA was performed for B, E, and $\mathbf{F}$, followed by post hoc 1-tailed $t$ tests. Nonparametric Kruskal-Wallis test was used for multiple group comparisons in C, followed by post hoc 1-tailed Mann-Whitney $U$ test. Two-way ANOVA for treatment $\times$ time interaction performed in $\mathbf{D}$ and $\mathbf{G}$, followed by post hoc unpaired $t$ test. $P=0.008$ (D); $P=0.52$ (G). All data shown as mean \pm SEM; $n$ indicated within bars. mation (12, 25-27). Quantification of myoinositol in the corpus callosum showed a distinct genotype difference and progression of inflammation over time in $\mathrm{Cnp}^{-/-}$mice (Figure 3B). Upon PLX5622, the progressive inflammatory phenotype of $\mathrm{Cnp}^{-/-}$mice was reduced to nearly the level seen in WT (Figure 3, C and D). A similar but less prominent effect of PLX5622 was observed in the cortex, where the MRS-detectable neuroinflammation in $\mathrm{Cnp}^{-1}$ mice was also less pronounced in treated mice (Figure 3, E-G).

Catatonia and IHC markers of brain inflammation and neurodegeneration in 6-month-old Cnp $\mathrm{p}^{-1}$ mice are reduced by PLX5622 treatment. The encouraging results of the prevention study made us wonder whether similar effects of microglia depletion by CSF1R inhibition could be observed at a more progressed disease state with advanced neurodegeneration (14) (Figures 4, A-F, and Figure 5, A-G). As expected, $C n p^{-/-}$mice at the age of 27 weeks were catatonic (Figure 5G). After 5 and 8 weeks of PLX5622, catatonic signs were reduced (Figure $4 \mathrm{~B}$ ). In agreement with the literature, PLX5622-treated WT mice were almost completely depleted of Iba1 ${ }^{+}$cells $(22,23)$, even though - compared with young mice - around 10 times more microglial cells $/ \mathrm{mm}^{2}$ corpus callosum area were retained. In contrast, $\mathrm{Cnp}^{-/-}$mice showed substan- tially reduced microglia numbers upon PLX5622 administration to one-third of untreated $\mathrm{Cnp}^{-/-}$mice, but just reached untreated WT levels (Figure 4C). This finding may imply that upon aging and/or in situations of strong inflammatory stimulation, a considerable number of microglia lose their responsiveness to CSF1R inhibition. CD68 immunostaining, localized to the lysosomal membrane and used as an additional readout of microglia activation that is upregulated in actively phagocytic cells (28), displayed a pattern very similar to that of Iba1 (Figure 4, D-F). Interestingly, quantification of microglia in the neighboring cingulate cortex as a crucial part of the prefrontal cortex also revealed a distinct increase in Iba1 ${ }^{+}$cells in $\mathrm{Cnp}^{-/-}$mice (Figure $2 \mathrm{E}$ and Figure $4 \mathrm{E}$ ). This demonstrates that white matter inflammation in mutants spreads onto gray matter areas where PLX5622 again leads to a considerable reduction in $\mathrm{Iba}^{+}$cells.

Both the remarkably increased axonal swellings $\left(\mathrm{APP}^{+}\right)$and the strongly enhanced $\mathrm{GFAP}^{+}$area seen in untreated $\mathrm{Cnp}^{-/}$mice were diminished under PLX5622, but remained greater than that seen in WT (Figure 5, A and B). The same holds true for $\mathrm{CD}^{+} \mathrm{T}$ lymphocytes, which are attracted by chemokines and cytokines into the inflammatory brain and were also considerably reduced upon 
A

Treatment study design: CSF1 receptor inhibition

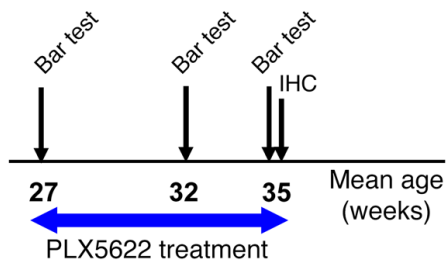

IHC: 8 weeks of continuous PLX5622
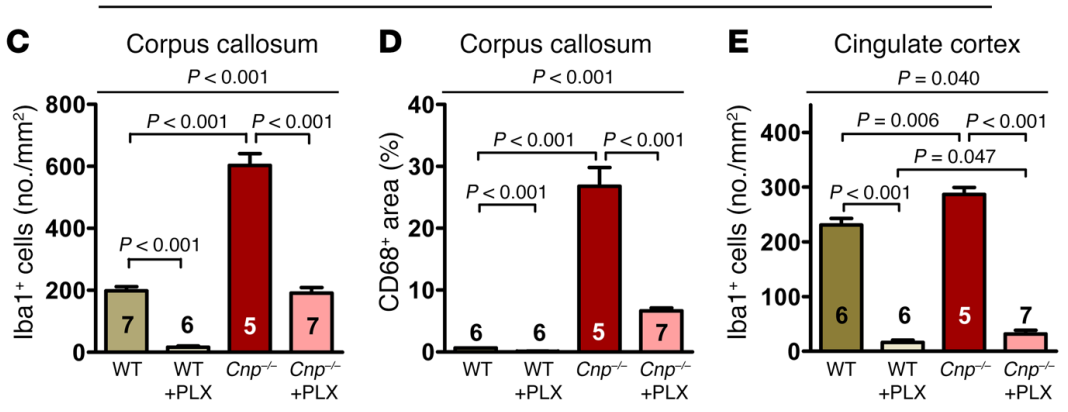

$\mathbf{F}$

IHC: corpus callosum upon 8 weeks of continuous PLX5622

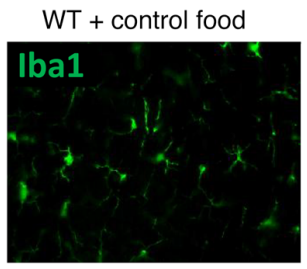

\section{WT + PLX5622}
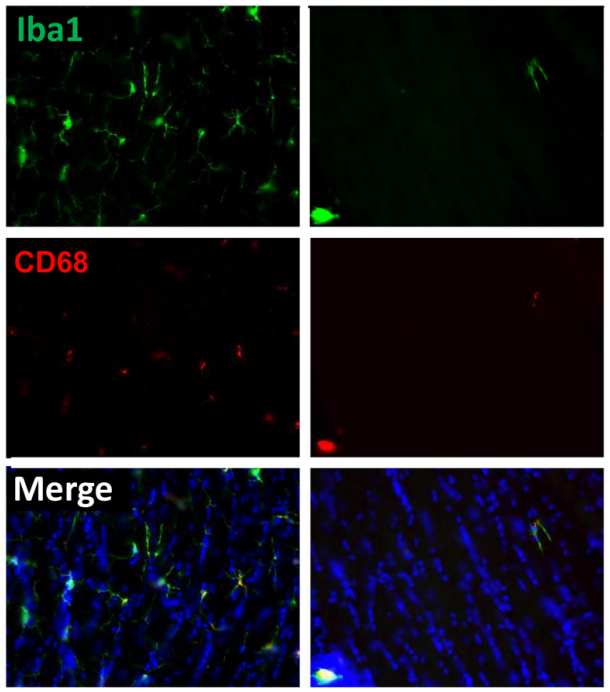

B Catatonic signs after 5 and 8 weeks on PLX5622

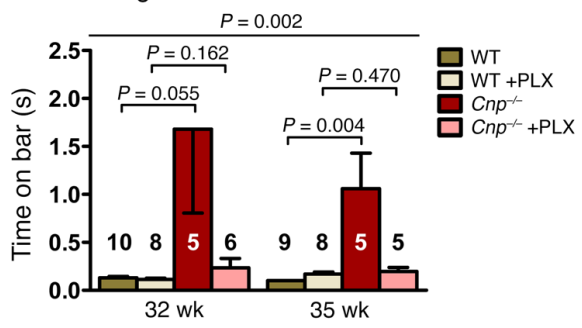

Figure 4. Catatonia and white matter inflammation in Cnp mutant mice and their treatment by CSF1R inhibition: Part I. (A) Schematic overview of the treatment study design, including PLX5622 (versus regular diet) feeding phase (blue arrow) and time points/age of testing/analyses (black arrows). (B) Catatonic signs in WT and $\mathrm{Cnp}^{-/-}$mice after 5 and 8 weeks on PLX5622 or control diet. (C and D) IHC quantifications in the corpus callosum (as shown in Figure 2E) at the age of 35 weeks after 8 weeks of PLX5622 or control diet: lba1+ cells (no./mm²; 1 section/brain) and $\mathrm{CD68}^{+}$area (\%; 1 section/brain). (E) Iba $1^{+}$cells in the cingulate cortex (no./mm²; 1 section/brain; area described in Figure 2E). (F) Representative IHC images illustrating the results in $\mathbf{C}$ and $\mathbf{D}$. All data in $\mathbf{B}$ C, D, and $\mathbf{E}$ were individually tested for Gaussian distribution using the Kolmogorov-Smirnov test. Nonparametric Kruskal-Wallis test was performed in B for multiple group comparisons, followed by post hoc 1-tailed Mann-Whitney $U$ test. Two-way ANOVA was performed for $\mathbf{C}, \mathbf{D}$, and $\mathbf{F}$, followed by post hoc 1-tailed unpaired $t$ test. All data are shown as mean \pm SEM; $n$ indicated within bars.
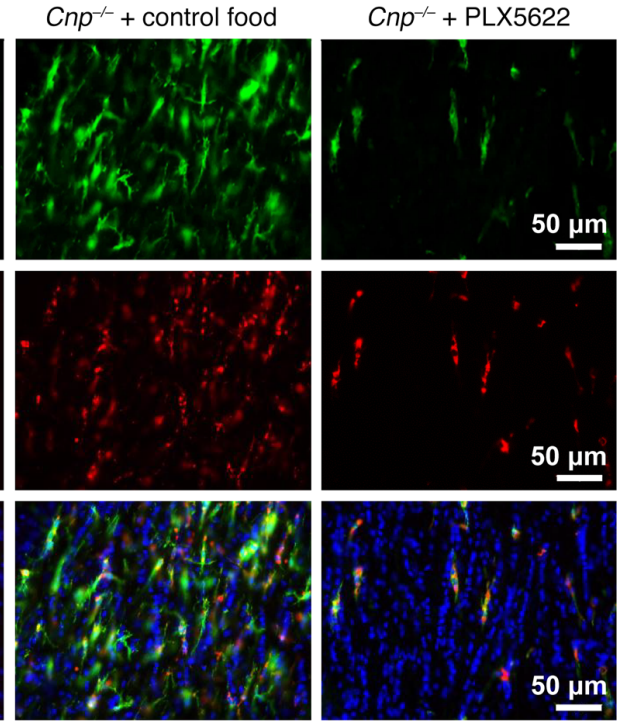

PLX5622 treatment (Figure 5, C and D). Since the abundance of oligodendrocyte precursor cells is known to be influenced by microglia and CSF1R inhibition during postnatal development was shown to decrease their numbers (29), PDGFR $\alpha$ staining was also performed. Indeed, $\mathrm{Cnp}^{-/-}$mice, with their elevated numbers of PDGFR $\alpha^{+}$oligodendrocyte precursors, as well as WT mice respond to PLX5622 with a reduction of PDGFR $\alpha^{+}$cells to nearly $50 \%$ (Figure $5, \mathrm{E}$ and F).

Other myelin mutants with neuroinflammation also develop catatonia. Finally, we asked whether and when other mouse mutants exhibiting subtle myelin abnormalities and mild neuroinflammation later in life would likewise display a catatonic phenotype. This included mice heterozygous for $M b p$ (12) and $P l p$-null mutant mice 44
(30). Behavioral testing began at 8 weeks of age and was repeated every 4 to 6 weeks. Indeed, we detected signs of catatonia in heterozygous $M b p^{+/-}$mice at ages 60 to 72 weeks, similar to those seen in the aging phenotype of $C n p^{+/-}$mice (11). Hemizygous $P l p^{-/ y}$ mice displayed catatonic signs already at 25 weeks of age (Figure $5 \mathrm{G}$ ). We note, however, that in all of these mouse lines, including Cnp mutants, and similarly across the different age groups, catatonic signs show some variability regarding time on the bar. While as a group, catatonic mice clearly differ from WT mice, the severity range is sometimes considerable.

To test for specificity of catatonic signs as a white matter problem, the bar test was performed at various ages in a wide range 


\section{Project I}

Downloaded from http://www.jci.org on December 19, 2017. https://doi.org/10.1172/JCI97032

The Journal of Clinical Investigation

RESEARCH ARTICLE

IHC: 8 weeks of continuous PLX5622 - corpus callosum

A

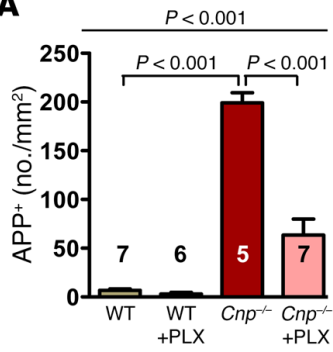

B

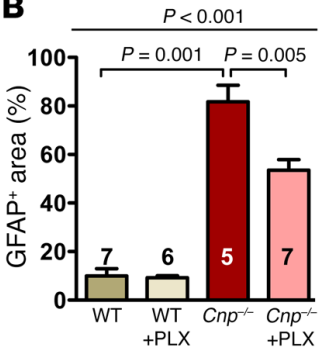

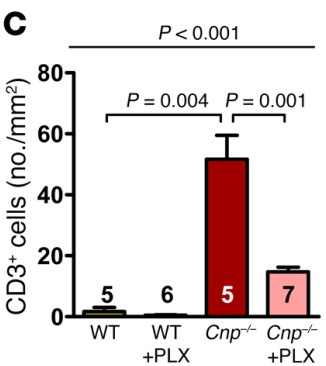

D

WT

$\mathrm{Cnp}^{-1}$
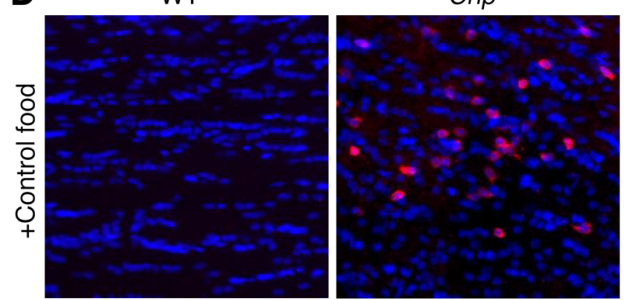

E

WT
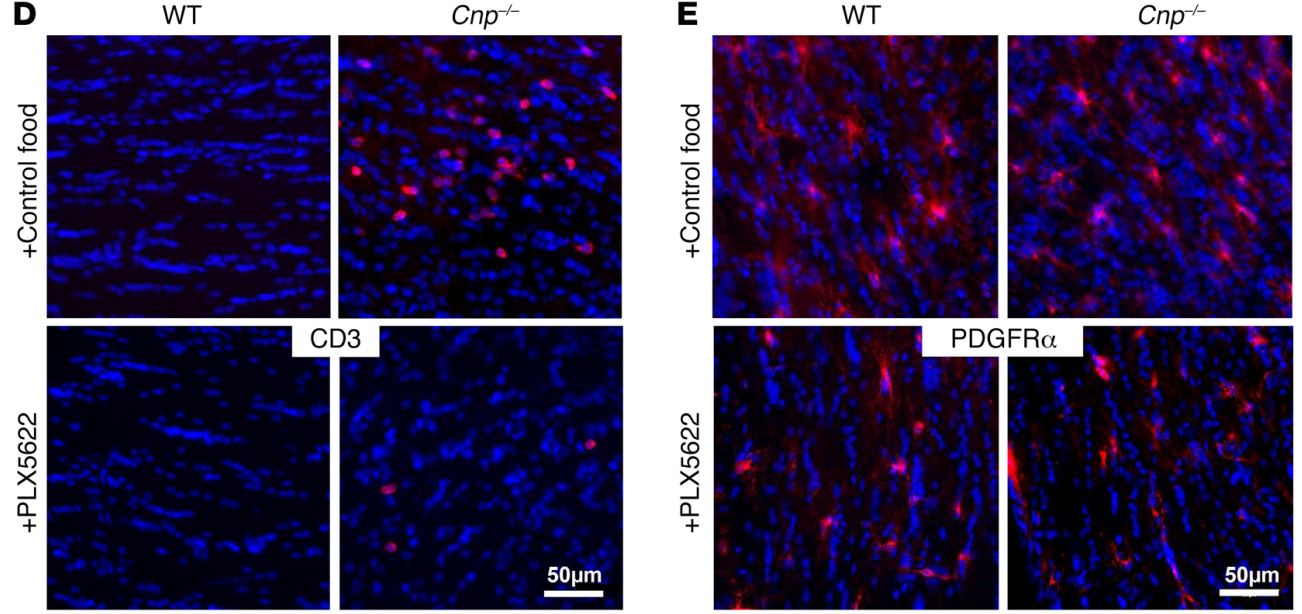

CD3
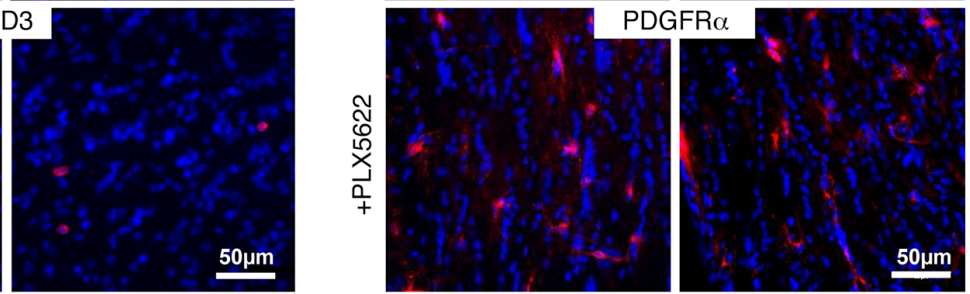

$\mathbf{F}$

G

Catatonic signs in other myelin mutants
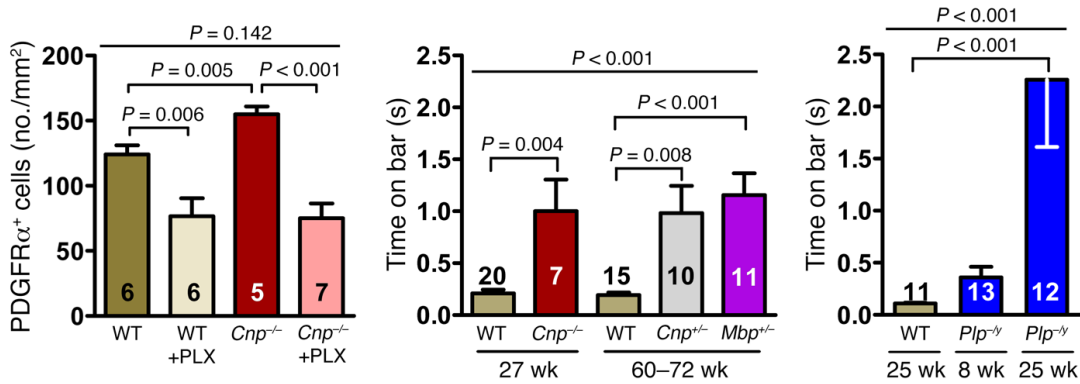

Figure 5. Catatonia and white matter inflammation in Cnp mutant mice and their treatment by CSF1R inhibition: Part II. (A) APP' swellings (indicating $\mathrm{APP}^{+}$spheroids; no./mm²; 3 sections/brain) and (B) densitometric analysis of GFAP ${ }^{+}$area (\%; indicating astrogliosis; 1 section/brain). (C) $\mathrm{CD}^{+}$cells (no./ $\mathrm{mm}^{2}$; indicating T lymphocyte invasion; 1 section/brain). ( $\mathbf{D}$ and $\left.\mathbf{E}\right)$ Representative IHC images illustrating CD3 and PDGFR $\alpha$ staining. (F) PDCFR $\alpha^{+}$cells (no./mm²; indicating oligodendrocyte precursors; 1 section/brain). (C) Catatonic signs in WT, Cnp ${ }^{-1 /}$ (age of onset at 8 weeks of age, shown in Figure 2A), and $\mathrm{Cnp}^{+-}$mice compared with mice with mutations in other myelin-related genes $\left(\mathrm{Mbp}^{+/}\right.$, $\left.\mathrm{Pl} \mathrm{p}^{-/ \mathrm{y}}\right)$. Age of onset of catatonia in heterozygous mice $\left(\mathrm{Cnp}^{+-}\right.$, $\mathrm{Mbp}^{+-}$) seen at around 60-72 weeks and in Plp ${ }^{-/ y}$ mice at 25 weeks. All data in $\mathbf{A}, \mathbf{B}, \mathbf{C}, \mathbf{F}$, and $\mathbf{G}$ were individually tested for Gaussian distribution using the Kolmogorov-Smirnov test. Nonparametric Kruskal-Wallis test was performed for B, C, and $\mathbf{G}$ for multiple group comparisons, followed by post hoc 1-tailed Mann-Whitney $U$ test. Two-way ANOVA was performed for $\mathbf{A}$ and $\mathbf{F}$, followed by post hoc 1-tailed unpaired $t$ test. All data are shown as mean \pm SEM; $n$ indicated within bars.

of CNS mutants with reported behavioral phenotypes relevant for other facets of neuropsychiatric disease, e.g., autistic, cognitive, or metabolic syndromes. This included mutations of synaptic genes Nlgn $4^{-/-}$(31), Caps1 ${ }^{-/}$(32), Psd93\%, and Psd $95^{-/-}$(33), autophagy dysfunction in $\mathrm{Ambra1}^{+/-}$mutants (34), and $\mathrm{ApoE}^{-/-}$mice with a disturbed blood-brain barrier (35), yet none of these control mice revealed catatonic signs (all $P \geq 0.2$ compared with respective
WT). In addition, normal WT (C57BL/6N) mice were tested at 1 , 6,10 , or 14 weeks after a single injection of high-dose LPS $(5 \mathrm{mg} /$ $\mathrm{kg}$ i.p. given at the age of 4 months), known to induce persistent microgliosis (36). Also here, no catatonic signs were observed (all $P \geq 0.2$ compared with respective untreated controls). Interestingly, even catatonic $P l p^{-/ y}$ mice did not reveal any further increase in their catatonic signs upon LPS (5 mg/kg i.p.) when tested at 1 , 


\section{Project I}

Downloaded from http://www.jci.org on December 19, 2017. https://doi.org/10.1172/JCI97032

RESEARCH ARTICLE

The Journal of Clinical Investigation

4,8 , or 12 weeks after a single injection (all $P \geq 0.8$ compared with PBS-injected controls), suggesting that just the myelin-associated inflammation is critical for catatonic signs.

\section{Discussion}

We have identified altered myelin gene expression and minor structural abnormalities of CNS myelin as the trigger of an inflammatory response predominantly in white matter tracts and an underlying cause of catatonic signs in the behavior of mice and humans. This provides a mechanistic insight into a previously enigmatic neuropsychiatric phenotype and expands our view on the role of white matter integrity in cognitive and executive functions in general. Importantly, we have discovered a potential therapy by targeting microglial cells, which emerge as mediators of this neuropsychiatric syndrome.

Our study was based on the previously reported "catatoniadepression" phenotype of aged mice heterozygous for Cnp (11), which led us to a translational approach from mice to humans and back. Studying the deeply phenotyped GRAS sample of schizophrenic patients $(37,38)$, we first demonstrated an age-dependent, unexpectedly high prevalence of more than $25 \%$ of catatonic signs, exceeding by far the current estimates of approximately $0.2 \%-3.0 \%$ catatonia in schizophrenic subjects (5). This major discrepancy is likely explained by the often-missed clinical diagnosis, in particular of the milder forms that are much more common and classified as neurological soft signs (8).

Importantly, we noticed that the more severe catatonic signs of GRAS patients are associated with rs2070106-AA, a CNP partial loss-of-function genotype $(13,39,40)$, a finding that we replicated in an independent schizophrenia cohort. We could further show by MRI in a general population sample that CNP rs2070106AA subjects were more likely than G carriers (GG or AG) to display WMH in frontal and temporal brain areas as well as in deep brain structures. These subclinical findings are not unusual in healthy individuals, where they have been associated with vascular changes, demyelination, and activated microglia (19-21). Even though this literature is suggestive, we have of course no direct proof (e.g., brain biopsies) that inflammation is increased in white matter tracts of live human AA carriers. We note, however, that diffusion tensor imaging identified higher axial diffusivity and a higher apparent diffusion coefficient in the frontal part of the corpus callosum of AA as compared with GG subjects, consistent with a more progressed axonal loss/degeneration (11). This finding further supports the presence of at least low-grade inflammation in AA individuals.

While WMH in humans may be an indirect indicator of white matter inflammation, presence of the latter in $\mathrm{Cnp}^{-/-}$mice and its reduction upon microglia depletion were directly shown in the present study. In fact, since microgliosis is a feature of Cnp mutant mice (14) and aged Cnp heterozygotes exhibit a catatonia-depression phenotype together with late-onset brain inflammation (11), we tested our hypothesis that neuroinflammation itself is causal for the catatonic signs of myelin mutant mice. We chose to treat Cnp-null mutant mice at an age at which they were still free of motor impairments, and indeed, we could completely prevent catatonia onset in these young animals by depleting microglia via administration of the CSF1R inhibitor PLX5622 (22, 23, 41) imme- diately after weaning. The CSF1R inhibitor also proved effective for treatment of existing catatonia in older $\mathrm{Cnp}^{-/}$mutants, in which it caused a reduction (but not prevention) of axonal degeneration. This is in line with CNP deficiency as a "driver" of neurodegeneration following traumatic brain injury (42). However, the complete prevention of catatonic signs in the young Cnp-null mutants strongly suggests that catatonia is not caused by axonal degeneration but is primarily a "microglial disease" induced by mild myelin perturbations. This conclusion is also supported by the catatonic phenotype in aged heterozygous $M b p$ mice that exhibit microglial activation (12), but will not develop the axonal degeneration phenotype of Plp- and Cnp-null mutant mice with motor impairments $(14,43)$.

We note that catatonic signs in mice are presently measured by the bar test only, which requires an experienced examiner to yield reproducible results. Having to build on a single readout may explain the cohort-to-cohort variation in the expression severity of this phenotype, which does not show consistent worsening over time (compared with WT controls). Thus, catatonic signs constitute a dichotomous variable in mice (yes/no) rather than a continuous one, as in humans. In fact, several readouts underlie the severity rating used here for humans ( 9 subtests of Cambridge Neurological Inventory [CNI], including gait mannerisms, gegenhalten, mitgehen, imposed posture, abrupt, or exaggerated spontaneous movements, iterative movements, automatic obedience, and echopraxia; ref. 8). To obtain a similarly robust continuous measure for mice, more catatonia tests are presently being developed in our laboratory, but these tests still require replications in independent mouse cohorts and extensive crossvalidation with bar test results. We hope to ultimately provide a catatonia severity composite score for mice - as we previously established for autistic phenotypes (44) - which will then help diminish sample heterogeneity as typically obtained for single tests.

Interestingly, treating older Cnp mutants with PLX5622 was less effective in eliminating microglial cells. Thus, at least a subpopulation of microglia seems to become unresponsive to CSF1R inhibition in the course of neurodegeneration. This represents a therapeutically relevant observation that may reflect the activation status of these cells and deserves further investigation. Based on our data, we cannot exclude that some of these resilient cells are invading peripheral macrophages, known to be $\mathrm{Iba1}^{+}$and perhaps resistant to PLX5622, or that a higher dose of PLX5622 would have eliminated even those seemingly less responsive cells. The fact, however, that in our pilot experiments with older WT mice, a higher cumulative dose (8-week treatment versus 5-week treatment) had not resulted in any stronger depletion may point against this interpretation.

We point out that CSF1R inhibition also affects cells other than microglia, which may have contributed to the catatonic phenotype (45). Upon CSF1R inhibition, we saw not only a decline in CD3 ${ }^{+}$ $\mathrm{T}$ cells that are attracted to the brain by the inflammatory milieu and may influence microglial behavior, but also a decrease in the $\mathrm{GFAP}^{+}$area as a measure of robust astrogliosis. In addition, we noted a diminished number of PDGFR $\alpha^{+}$oligodendrocyte precursors following CSF1R inhibition, similar to what was shown earlier during brain development (29). We thus have to assume that indirect effects could add to the therapeutic benefit of PLX5622. 


\section{Project I}

Downloaded from http://www.jci.org on December 19, 2017. https://doi.org/10.1172/JCl97032

A large number of studies in mouse models of neuropsychiatric disease have analyzed the contributions of single genes and developmental defects on cognitive dysfunction, autistic traits, signs of depression, and other mental disease-relevant phenotypes (46-49). However, this research has mainly focused on negative and cognitive symptoms, which are easier to model in mice than any of the positive symptoms (delusions, hallucinations), most of which are considered human specific. Catatonia, defined in DSM- 5 as a disease specifier for schizophrenia and major mood disorders (5), emerges as an intriguing exception, a positive symptom, and quantifiable readout that can be studied across species.

Our study is, to our knowledge, the first molecular-genetic approach to catatonia and catatonic signs. However, we have to assume that the etiology of these conditions might be heterogeneous. Myelin perturbations may be just one of several possible causes. Further studies are needed to determine whether secondary neuroinflammation is always essential in the "final common pathway" to catatonia. The inefficiency, however, of LPS-mediated brain inflammation to induce catatonic signs in WT mice or to further enhance them in catatonic $P l p^{-/ y}$ mutants supports the concept of primary myelin alterations (or other underlying causes) being of critical importance for the development of a catatonic phenotype. In fact, peripheral LPS injection most likely acts by causing the liver to produce high levels of circulating TNF- $\alpha$, which enters the brain at specific sites to incite the abnormal stimulation of endogenous microglia and trigger a vicious and long-lasting circle of events that may even lead to neurodegeneration $(50,51)$. Here, gray matter areas, such as dopaminergic nuclei, seem to be at higher risk than white matter, whereas myelin-associated inflammation is a unique feature of $\mathrm{Cnp}^{-/-}$and other myelin mutant mice.

The sudden loss of motor control in catatonia, followed by an equally sudden regaining of control - often within seconds - ultimately suggests a dysfunction of synaptic circuitry. Importantly, we could show that chronic neuroinflammation in the subcortical white matter progresses into the cingulate cortex, where activated microglia are known to perturb normal synaptic function (16). The production of nitric oxide and axonal conduction blocks, for instance, constitute well-established links between activated microglia and neurodegeneration (52). Within the cortex, the inflammation associated with myelinated fibers may also affect nonmyelinated axons and dendrites (as bystanders), but is probably rather transient or fluctuating because neuronal somata (unlike axons) and the synaptic circuitry are more likely to recover from acute mitochondrial perturbations caused by reactive oxygen species such as $\mathrm{NO}$, as seen in chronic progressive multiple sclerosis (53).

Inflammation, identified in the present study as a major mechanism of catatonic signs, might also affect other phenotypes of mental disease, which could explain the frequently observed fluctuations in their clinical presentation ("episodes"). Alteration of the body's inflammatory state, as encountered in infectious diseases or even during the normal estrous cycle (luteolysis), may have an additional amplifying impact on inflammation within the CNS and thus contribute to the still poorly explained episodic course of many neuropsychiatric diseases. Also, the most severe acute form of catatonia, the life-threatening febrile pernicious catatonia (4), may represent a fulminant inflammation of white matter tracts.
Catatonia in Cnp mutant mice was prevented by depletion of microglia at a young age and was even treatable in older animals. While this is important as a proof-of-principle, more research will be needed to define effects of repeated treatment cycles with CSF1R inhibitors as well as to delineate more specific pharmacological targets in activated microglia. In fact, repeated treatment cycles with treatment-free intervals may still be effective, but reduce the risk of side effects, potentially resembling hereditary diffuse leukoencephalopathy with spheroids (HDLS), a CNS white matter disease described in individuals with loss-of-function mutations of the CSF1R gene (54). Catatonic signs in patients with schizophrenia are generally mild and per se may not even require specific treatments. However, they likely constitute a surrogate marker for disturbance of broader executive functions and for cognitive deficits, which are severely disabling and currently untreatable, but may also be responsive to specific microglia-targeting antiinflammatory therapies.

\section{Methods}

\section{Human studies}

GRAS sample of schizophrenic subjects. The GRAS data collection (37, 38) involved deeply phenotyped patients $(n=1095$, age $39.1 \pm 12.7$ years; $66.8 \% \mathrm{men}$ ) diagnosed with schizophrenia or schizoaffective disorder according to DSM-IV-TR (55). Catatonic signs as the present study's target phenotype are from the CNI (8). Genotyping of CNP SNP rs2070106 was performed using a semi-custom Axiom MyDesign Genotyping Array (Affymetrix) as reported previously $(35,56)$.

Würzburg replication sample. Schizophrenic subjects $(n=288$, age $41.4 \pm 13.5$ years; $54.5 \%$ men) were assessed categorically into catatonia versus no catatonia according to Leonhard's classification (24), as described earlier (57). Genotyping of CNP SNP rs2070106 was performed by means of a quantitative reverse-transcriptase PCR-based (qRT-PCR-based) system using a custom-made primer (KASP assay, LGC Genomics).

SHIP general population sample. A subsample $(n=552$, age 46.2 \pm 11.4 years; $42.6 \%$ men) of the baseline cohort of SHIP-TREND-0 (accessible via application at www.community-medicine.com) was analyzed (58). Only individuals with available SNP information, valid brain MRI scans, complete covariate data, and no neurological conditions were included. Genotyping was performed using the Illumina HumanOmni2.5-Quad and imputation of genotypes via IMPUTE v.2.1.2.3 against the HapMap II (CUv22, Build36) reference panel. The call rate was very high for the CNP SNP rs2070106 (1.00).

MRI acquisition. T1- and T2-weighted MRI were used to measure regional patterns of WMH. All images were obtained using a 1.5T Siemens MRI scanner (Magnetom Avanto, Siemens Medical Systems) with an axial T1-weighted MPRAGE sequence and the following parameters: $1 \mathrm{~mm}$ isotropic voxels (flip-angle $15^{\circ}$ ); $3.37 \mathrm{~ms}$ echo time; $1900 \mathrm{~ms}$ repetition time, and $1100 \mathrm{~ms}$ inversion time. Axial T2-FLAIR sequence had the following parameters: $0.9 \times 0.9 \mathrm{~mm}$ in-plane spatial resolution; $3.0 \mathrm{~mm}$ slice thickness (flip-angle $15^{\circ}$ ); $325 \mathrm{~ms}$ echo time; $5,000 \mathrm{~ms}$ repetition time. An automated multimodal segmentation algorithm for WMH determination produced a probabilistic map, thresholded to generate a binary image. Thresholding was based on the default threshold value obtained from algorithm training data. To calculate WMH volume within specific ROI, we applied a multiatlas 


\section{Project I}

Downloaded from http://www.jci.org on December 19, 2017. https://doi.org/10.1172/JCI97032

RESEARCH ARTICLE

The Journal of Clinical Investigation

segmentation method. This included nonlinear registration of multiple atlases with ground-truth labels for every individual scan. Finally, WMH was determined for every region of the brain by masking WMH from all other regions (20).

\section{Mouse studies}

In all experiments, the experimenter was unaware of mouse genotypes and treatments (fully blinded).

Mouse maintenance and genotyping. Male and female mice were group-housed separately in ventilated cabinets (Scantainers; Scanbur Karlslunde) unless otherwise indicated for experiments requiring single housing. Mice were maintained on a 12-hour light/12-hour dark cycle (lights off at $7 \mathrm{pm}$ ) at $20-22^{\circ} \mathrm{C}$, with access to food and water ad libitum, woodchip bedding, and paper tissue as nesting material. Mutant mice (all C56BL/6J background) were genotyped as previously described for $C n p(11,14), M b p$ (12), and Plp (30). Only males were used for experiments with $M b p$ and $P l p$ mutants, while both females and males were used for experiments with Cnp mutants (housing and testing were always separated). We did not observe any gender differences in catatonic signs. The following neuropsychiatric phenotype-relevant mouse lines, with mutations affecting synapses, blood-brain barrier function or autophagy, were used as controls:

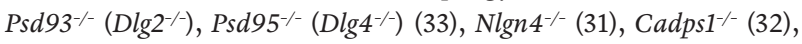
ApoE - $^{-1}$ (35), and $\mathrm{Ambra1}^{+/-}$(34).

Treatments. PLX5622 (formulated in AIN-76A standard chow by Research Diets; 1,200 ppm) and control food (AIN-76A) were provided by Plexxikon Inc. $(22,23)$. For tracking of potential batch-tobatch variations in future studies, PLX5622 lot numbers are given: 17032710A5TT1.0i; 16010809A9TT1.0i; 17010309A7TT1.0i; and 16092608A1TT1.0i. LPS was injected intraperitoneally ( $5 \mathrm{mg} / \mathrm{kg}$ LPS; O111:B4; Sigma-Aldrich).

Bar test for catatonia. All mice were transferred to the experimental room 30 minutes prior to testing for habituation. The bar test was performed as previously described $(11,59)$. Briefly, the mouse was gently carried by the tail to a horizontal bar made of stainless steel (12 cm length, $2.5 \mathrm{~mm}$ diameter). Upon grasping the bar with both forepaws, the mouse was moved downwards so that its hind paws had contact with the floor before its tail was released. All experiments were recorded using a high-resolution camcorder (Sony HDR-CX405, Sony Europe Limited). Catatonic signs were scored manually from video recordings as the duration of uninterrupted time a mouse stood nonmoving with at least 1 forepaw on the bar and both hind paws on the ground. Scoring was performed by trained observers blinded to treatments and genotypes (for illustration, see Supplemental Video 1).

Immunohistochemistry. Anesthetized mice were perfused with Ringer's solution (Braun) followed by $4 \%$ formaldehyde. Brains were collected, postfixed overnight in $4 \%$ formaldehyde, cryoprotected in $30 \%$ sucrose, and stored at $-80^{\circ} \mathrm{C}$. Whole mouse brains were cut into 30- $\mu \mathrm{m}$-thick coronal sections on a cryostat (Leica, CM1950) and kept in storage solution ( $25 \%$ ethylene glycol/25\% glycerol in PBS) at $-20^{\circ} \mathrm{C}$. For APP and CD3 staining, sections were microwaved in citrate buffer (1 mM, pH 6.0) and for APP detection further pretreated in $3 \%$ $\mathrm{H}_{2} \mathrm{O}_{2}$. All sections were permeabilized and blocked with 5\% normal horse serum (NHS)/0.5\% Triton X-100 in PBS for 1 hour at room temperature. Sections at a bregma level between +1.15 and $+0.5 \mathrm{~mm}$ were immunostained for Iba1 (rabbit, 1:1,000, catalog 019-19741,
Wako), GFAP (mouse, 1:500, catalog NCL-GFAP-GA5, NovocastraLeica), CD3 (rat, clone CD3-12, 1:100, catalog MCA1477, Bio-Rad), CD68 (rat, 1:400, catalog MCA1957GA, Bio-Rad), PDGFR $\alpha$ (rabbit, 1:300, catalog 3174, Cell Signaling), or APP (mouse, 1:850, catalog MAB348, Chemicon-Millipore) in 3\% NHS/0.5\% Triton X-100 in PBS over 2 nights at $4^{\circ} \mathrm{C}$. For fluorescent microscopy, 1 hour incubation at room temperature with donkey anti-rabbit Alexa Fluor 647 (catalog A-31573), donkey anti-mouse Alexa Fluor 488 (catalog A-21202), goat anti-rat Alexa Fluor 647 (catalog A-21247), or goat anti-rabbit Alexa Fluor 555 (catalog A-21428) antibodies (1:1,000; Thermo Fisher Scientific-Life Technologies) in 3\% NHS/0.5\% Triton X-100 in PBS was used. For DAB-based immunostaining, biotinylated horse anti-mouse antibody (1:200; Vector Laboratories) in 3\% NHS/0.5\% Triton X-100 in PBS and subsequent Vectastain Elite ABC Kit (Vector Laboratories) were used according to the manufacturer's instructions. Cell nuclei were counterstained with DAPI (1:5,000, Sigma-Aldrich) or Mayer's hemalum solution (Merck).

Morphometry. For the analysis of Iba1, GFAP, CD3, CD68, and PDGFR $\alpha$ fluorescent staining, brain slices were scanned using an inverted epifluorescent microscope with a $20 \times$ /NA0.4 or $40 \times$ / NA0.6 objective lens (Leica, DMI6000B) and quantified using Fiji software (http://fiji.sc/Fiji). Corpus callosum or cingulate cortex (Cg1 and Cg2) of each brain was defined on a DAPI channel as ROI. $\mathrm{Iba}^{+} \mathrm{DAPI}^{+}, \mathrm{CD}^{+} \mathrm{DAPI}^{+}$, and $\mathrm{PDGFR} \alpha^{+} \mathrm{DAPI}^{+}$cells were manually counted and density calculated with normalization to ROI. GFAP ${ }^{+}$ and $\mathrm{CD} 68^{+}$regions were quantified upon uniform thresholding with the respective area expressed as percentage of corpus callosum. $\mathrm{APP}^{+}$swellings were manually counted using a light microscope (Olympus BX-50) connected to a computer-driven motorized stage, $\mathrm{z}$-axis position encoder (microcator), and a microfire video camera interfaced to a PC using Stereo Investigator 6.55 software (MicroBrightfield Inc.). Representative images of $\mathrm{APP}^{+}$swellings were taken with a light microscope with a $100 \times /$ NA 1.30 oil objective lens (Zeiss Imager Z1).

MRI and ${ }^{1} H$-MRS. Mice were anesthetized with $5 \%$ isoflurane, intubated, and kept at $1.75 \%$ isoflurane by active ventilation with a constant respiratory frequency of 85 breaths/min (Animal Respirator Advanced, TSE Systems). MRI and localized ${ }^{1} \mathrm{H}$-MRS were performed at a magnetic field strength of 9.4T (Bruker BioSpin). MRI consisted of T2-weighted images (2D-FSE, TR/TE $=2800 / 11 \mathrm{~ms}, 100 \times 100 \times$ $300 \mu \mathrm{m}^{3}$ ) based on which respective volumes of interest for localized proton-MR spectra were positioned. MR spectra (STEAM, TR/TE/ $\mathrm{TM}=6,000 / 10 / 10 \mathrm{~ms}$ ) were obtained from a volume of interest in the cortex $\left(3.9 \times 0.7 \times 3.2 \mathrm{~mm}^{3}\right)$ and corpus callosum $\left(3.9 \times 0.7 \times 1.7 \mathrm{~mm}^{3}\right)$. Metabolite quantification was completed with spectral evaluation by LCModel (Version 6.3-1L). Results with Cramer-Rao lower bounds greater than $20 \%$ were excluded from further analysis.

\section{Statistics}

Group differences for continuous variables in human samples were assessed using the Kruskal-Wallis and Jonckheere-Terpstra trend tests. Genotype comparisons used the $\chi^{2}$ test. Multivariate linear regression models were run with $\mathrm{WMH}$ volume as a dependent variable and rs2070106 genotype-age interaction term as a predictor of interest. WMH volumes were transformed via cubic root due to their highly skewed distributions (20). For whole brain, total WMH volume was calculated summing all clusters greater than $10 \mathrm{~mm}^{3}$ to 


\section{Project I}

Downloaded from http://www.jci.org on December 19, 2017. https://doi.org/10.1172/JCl97032

reduce noise, followed by hypothesis-driven (11) post hoc testing of WMH from 5 predefined ROI (frontal, temporal, parietal and, as control, occipital lobes, as well as deep structures). For sensitivity analyses, all models described above were rerun using bootstrap methodology (2,000 replications) to derive SEM and CI independently of parametric assumptions such as Gaussian distribution or homoscedasticity. No major differences in standard ordinary least squares results were found. For mouse statistics, data distribution and variance homogeneity were determined by Kolmogorov-Smirnov test and outliers via the Grubbs test (https:/graphpad.com/quickcalcs/ Grubbs1.cfm). Two-way ANOVA with/without repeated measures was used for normally distributed data. Kruskal-Wallis test was used for data without normal distribution. Between-group comparisons were performed by Student's $t$ test for dependent/independent samples or Mann-Whitney $U$ test. $P \leq 0.05$ was considered significant. All statistical analyses were performed using SPSS (v 17.0; IBMDeutschland GmbH), STATA14/MP (Stata Inc.), or Prism 5 software (GraphPad Software).

\section{Study approval}

The GRAS study was approved by the ethics committees of GeorgAugust-University and participating centers across Germany, complying with the Helsinki Declaration. The Würzburg replication sample was approved by the Würzburg University Ethics Committee. The baseline cohort of SHIP-TREND-O, conducted in Northeast Germany, was approved by the Greifswald University Ethics Committee. All subjects (and/or legal representatives) gave written, informed consent. All animal tests were approved by the local Animal Care and Use Committee (LAVES, Niedersächsisches Landesamt für Verbraucherschutz und Lebensmittelsicherheit, Oldenburg, Germany) in accordance with the German Animal Protection Law.

\section{Author contributions}

$\mathrm{HE}$ and KAN created the concept and designed and supervised the study. MM, JH, MH, RAB, MB, SL, SKS, GH, CD, HV, AR, HJG, and $\mathrm{HE}$ acquired, analyzed, and interpreted data for human studies. HJ, SA, EB, HP, BLW, KAN, SB, and HE acquired, analyzed, and interpreted data for mouse phenotyping. HJ, SA, EB, SG, UCG, HBW, KAN, and HE acquired, analyzed, and interpreted data for mouse genetics. HE and KAN drafted the manuscript. HE, KAN, MM, HJ, SA, and EB drafted display items. All authors read and approved the final version of the manuscript.

\section{Acknowledgments}

This work was supported by the Max Planck Society, the Max Planck Förderstiftung, the Deutsche Forschungsgemeinschaft (DFG) (CNMPB and SPP1757), EXTRABRAIN EU-FP7, and the Niedersachsen-Research Network on Neuroinfectiology (N-RENNT). SHIP is part of the Community Medicine Research Net of the University Medicine Greifswald, which is funded by the Federal State of Mecklenburg-West Pomerania. Genomewide data in SHIP have been supported by a joint grant from Siemens Healthineers, Erlangen, and the Federal State of Mecklenburg-West Pomerania. KAN holds a European Research Council Advanced Investigator grant. The authors thank all subjects for participating in the study, and all colleagues who have contributed over the past decade to GRAS data collection.

Address correspondence to: Klaus-Armin Nave or Hannelore Ehrenreich, Max Planck Institute of Experimental Medicine, Hermann-Rein-Strasse 3, 37075 Göttingen, Germany. Phone: 49.551.3899 ext 754; Email: nave@em.mpg.de (K.A. Nave). Phone: 49.551.3899 ext 628; Email: ehrenreich@em.mpg.de.
1. Nave KA, Werner HB. Myelination of the nervous system: mechanisms and functions. Annu Rev Cell Dev Biol. 2014;30:503-533.

2. Filley CM, Fields RD. White matter and cognition: making the connection. J Neurophysiol. 2016;116(5):2093-2104.

3. Nave KA, Ehrenreich H. Myelination and oligodendrocyte functions in psychiatric diseases. JAMA Psychiatry. 2014;71(5):582-584.

4. Fink M, Taylor MA. Catatonia: a clinician's guide to diagnosis and treatment. Cambridge, UK: Cambridge University Press; 2003.

5. Tandon R, et al. Catatonia in DSM-5. Schizophr Res. 2013;150(1):26-30.

6. Arora M, Praharaj SK. Butterfly glioma of corpus callosum presenting as catatonia. World J Biol Psychiatry. 2007;8(1):54-55.

7. Northoff G, et al. Orbitofrontal cortical dysfunction in akinetic catatonia: a functional magnetic resonance imaging study during negative emotional stimulation. Schizophr Bull. 2004;30(2):405-427.

8. Chen EY, et al. The Cambridge Neurological Inventory: a clinical instrument for assessment of soft neurological signs in psychiatric patients. Psychiatry Res. 1995;56(2):183-204.

9. Amir S. Catalepsy induced by body pinch: relation to stress-induced analgesia. Ann N Y Acad Sci. 1986;467:226-237.
10. Chaperon F, Thiébot MH. Behavioral effects of cannabinoid agents in animals. Crit Rev Neurobiol. 1999;13(3):243-281.

11. Hagemeyer $\mathrm{N}$, et al. A myelin gene causative of a catatonia-depression syndrome upon aging. EMBO Mol Med. 2012;4(6):528-539.

12. Poggi $G$, et al. Cortical network dysfunction caused by a subtle defect of myelination. Glia. 2016;64(11):2025-2040.

13. Peirce TR, et al. Convergent evidence for 2',3'-cyclic nucleotide 3'-phosphodiesterase as a possible susceptibility gene for schizophrenia. Arch Gen Psychiatry. 2006;63(1):18-24.

14. Lappe-Siefke C, et al. Disruption of Cnp1 uncouples oligodendroglial functions in axonal support and myelination. Nat Genet. 2003;33(3):366-374.

15. Chen SK, et al. Hematopoietic origin of pathological grooming in Hoxb8 mutant mice. Cell. 2010;141(5):775-785.

16. Hong S, Dissing-Olesen L, Stevens B. New insights on the role of microglia in synaptic pruning in health and disease. Curr Opin Neurobiol. 2016;36:128-134.

17. Parkhurst CN, et al. Microglia promote learning-dependent synapse formation through brain-derived neurotrophic factor. Cell. 2013;155(7):1596-1609.

18. Prinz M, Priller J. Microglia and brain macrophages in the molecular age: from origin to neuropsychiatric disease. Nat Rev Neurosci. 2014;15(5):300-312.

19. Fazekas F, et al. The morphologic correlate of incidental punctate white matter hyperintensities on MR images. AJNR Am J Neuroradiol. 1991;12(5):915-921.

20. Habes M, et al. White matter hyperintensities and imaging patterns of brain ageing in the general population. Brain. 2016;139(Pt 4):1164-1179.

21. Simpson JE, et al. Microglial activation in white matter lesions and nonlesional white matter of ageing brains. Neuropathol Appl Neurobiol. 2007;33(6):670-683.

22. Dagher NN, et al. Colony-stimulating factor 1 receptor inhibition prevents microglial plaque association and improves cognition in 3xTg-AD mice. J Neuroinflammation. 2015;12:139.

23. Elmore MR, et al. Colony-stimulating factor 1 receptor signaling is necessary for microglia viability, unmasking a microglia progenitor cell in the adult brain. Neuron. 2014;82(2):380-397.

24. Leonhard K. Classification of Endogenous Psychoses and Their Differentiated Etiology. Wien, Austria: Springer-Verlag Wien; 1999.

25. Badar-Goffer RS, Ben-Yoseph O, Bachelard HS, Morris PG. Neuronal-glial metabolism under depolarizing conditions. A 13C-n.m.r. study. Biochem J. 1992;282(Pt 1):225-230.

26. Chang L, Munsaka SM, Kraft-Terry S, Ernst T. 


\section{Project I}

Downloaded from http://www.jci.org on December 19, 2017. https://doi.org/10.1172/JCI97032

RESEARCH ARTICLE

Magnetic resonance spectroscopy to assess neuroinflammation and neuropathic pain. JNeuroimmune Pharmacol. 2013;8(3):576-593.

27. Ross BD, Bluml S, Cowan R, Danielsen E, Farrow $\mathrm{N}$, Gruetter R. In vivo magnetic resonance spectroscopy of human brain: the biophysical basis of dementia. Biophys Chem. 1997;68(1-3):161-172.

28. Zotova E, et al. Inflammatory components in human Alzheimer's disease and after active amyloid- $\beta 42$ immunization. Brain. 2013; 136(Pt 9):2677-2696.

29. Hagemeyer N, et al. Microglia contribute to normal myelinogenesis to oligodendrocyte progenitor maintenance during adulthood. Acta Neuropathol. 2017;134(3):441-458.

30. Klugmann M, et al. Assembly of CNS myelin in the absence of proteolipid protein. Neuron. 1997;18(1):59-70.

31. Jamain $\mathrm{S}$, et al. Reduced social interaction and ultrasonic communication in a mouse model of monogenic heritable autism. Proc Natl Acad Sci US A. 2008;105(5):1710-1715.

32. Jockusch WJ, et al. CAPS- 1 and CAPS-2 are essential synaptic vesicle priming proteins. Cell. 2007;131(4):796-808.

33. Winkler D, et al. Hypersocial behavior biological redundancy in mice with reduced expression of PSD95 or PSD93 [published online ahead of print February 9, 2017 ]. Behav Brain Res. https://doi. org/10.1016/j.bbr.2017.02.011.

34. Dere E, et al. Heterozygous ambra1 deficiency in mice: a genetic trait with autism-like behavior restricted to the female gender. Front Behav Neurosci. 2014;8:181.

35. Hammer C, et al. Neuropsychiatric disease relevance of circulating anti-NMDA receptor autoantibodies depends on blood-brain barrier integrity. Mol Psychiatry. 2014;19(10):1143-1149.

36. Qin L, Liu Y, Hong JS, Crews FT. NADPH oxidase and aging drive microglial activation, oxidative stress, and dopaminergic neurodegeneration following systemic LPS administration. Glia.
2013;61(6):855-868.

37. Begemann $\mathrm{M}$, et al. Modification of cognitive performance in schizophrenia by complexin 2 gene polymorphisms. Arch Gen Psychiatry. 2010;67(9):879-888.

38. Ribbe K, et al. The cross-sectional GRAS sample: a comprehensive phenotypical data collection of schizophrenic patients. BMC Psychiatry. 2010;10:91.

39. Iwamoto K, Ueda J, Bundo M, Nakano Y, Kato $\mathrm{T}$. Effect of a functional single nucleotide polymorphism in the 2',3'-cyclic nucleotide 3'-phosphodiesterase gene on the expression of oligodendrocyte-related genes in schizophrenia. Psychiatry Clin Neurosci. 2008;62(1):103-108.

40. Mitkus SN, et al. Expression of oligodendrocyteassociated genes in dorsolateral prefrontal cortex of patients with schizophrenia. Schizophr Res. 2008;98(1-3):129-138.

41. Waisman A, Ginhoux F, Greter M, Bruttger J. Homeostasis of microglia in the adult brain: review of novel microglia depletion systems. Trends Immunol. 2015;36 (10):625-636.

42. Wieser GL, et al. Neuroinflammation in white matter tracts of Cnp1 mutant mice amplified by a minor brain injury. Glia. 2013;61 (6):869-880.

43. Griffiths I, et al. Axonal swellings and degeneration in mice lacking the major proteolipid of myelin. Science. 1998;280(5369):1610-1613.

44. El-Kordi A, et al. Development of an autism severity score for mice using Nlgn 4 null mutants as a construct-valid model of heritable monogenic autism. Behav Brain Res. 2013;251:41-49.

45. Chitu V, Gokhan Ş, Nandi S, Mehler MF, Stanley ER. Emerging roles for CSF-1 receptor and its ligands in the nervous system. Trends Neurosci. 2016;39(6):378-393.

46. Lee YS, Silva AJ. The molecular and cellular biology of enhanced cognition. Nat Rev Neurosci. 2009;10(2):126-140.

47. Nestler EJ, Hyman SE. Animal models of neuropsychiatric disorders. Nat Neurosci.
The Journal of Clinical Investigation

2010;13(10):1161-1169.

48. Schroeder JC, Reim D, Boeckers TM, Schmeisser MJ. Genetic animal models for autism spectrum disorder. Curr Top Behav Neurosci. 2017;30:311-324.

49. Silverman JL, Yang M, Lord C, Crawley JN. Behavioural phenotyping assays for mouse models of autism. Nat Rev Neurosci. 2010;11(7):490-502.

50. Jeong HK, Jou I, Joe EH. Systemic LPS administration induces brain inflammation but not dopaminergic neuronal death in the substantia nigra. Exp Mol Med.2010;42(12):823-832.

51. Qin L, et al. Systemic LPS causes chronic neuroinflammation and progressive neurodegeneration. Glia. 2007;55(5):453-462.

52. Block ML, Zecca L, Hong JS. Microglia-mediated neurotoxicity: uncovering the molecular mechanisms. Nat Rev Neurosci. 2007;8(1):57-69.

53. Lassmann H, van Horssen J, Mahad D. Progressive multiple sclerosis: pathology and pathogenesis. Nat Rev Neurol. 2012;8(11):647-656.

54. Rademakers R, et al. Mutations in the colony stimulating factor 1 receptor (CSF1R) gene cause hereditary diffuse leukoencephalopathy with spheroids. Nat Genet. 2011;44(2):200-205.

55. American Psychiatric Association. Diagnostic statistical manual of mental disorders: DSM-IV-TR. Washington, DC: American Psychiatric Association; 2000.

56. Stepniak B, et al. Accumulated environmental risk determining age at schizophrenia onset: a deep phenotyping-based study. Lancet Psychiatry. 2014;1(6):444-453.

57. Reif A, et al. A neuronal nitric oxide synthase (NOS-I) haplotype associated with schizophrenia modifies prefrontal cortex function. Mol Psychiatry. 2006;11(3):286-300.

58. Völzke H, et al. Cohort profile: the study of health in Pomerania. Int J Epidemiol. 2011;40(2):294-307.

59. Kuschinsky K, Hornykiewicz O. Morphine catalepsy in the rat: relation to striatal dopamine metabolism. Eur J Pharmacol. 1972;19(1):119-122. 


\section{Chapter 3 \\ Project II}




\section{Project II}

\section{Isolated catatonia-executive dysfunction complex in aged mice induced by forebrain-specific loss of myelin integrity}

\section{Outline of the project}

In the framework of my first project we reported on a myelin gene-associated catatonic phenotype in $\mathrm{Cnp}^{-1}$ mice (Janova et al., 2018). Importantly, the phenotype was accompanied by neuroinflammation and -degenerative processes in white matter tracts and the cingulate cortex. Depletion of microglia at young and old age by usage of PLX5622 alleviated the phenotype on a behavioral and neuropathological level. Based on these findings, we aimed to characterize the phenotype of microglia undergoing depletion and upon repopulation in a subsequent study, and found catatonia and executive function to correlate in mice and men (Garcia-Agudo et al., 2019). The latter can be described as a complex cognitive function of higher-order (Diamond, 2013), even though historically diverse theories along with a respective battery of experimental approaches have been described (Chan et al., 2008). However, the importance of the frontal lobes and dominant functions of the PFC are well-accepted (Alvarez \& Emory, 2006; Chudasama, 2011; Yuan \& Raz, 2014). Knowing that Plp1 constitutive KO mice exhibit catatonic signs and impaired executive function in adulthood (Gould et al., 2018; Janova et al., 2018), I aimed to investigate the impact of neuroinflammation of frontal brain structures on these phenotypes, by application of cKO mice.

\section{(I) Immunohistochemical control of functionality of the mouse model}

In close collaboration with Prof. Klaus-Armin Nave, Ph.D., and PD Dr. Hauke B. Werner from the department of Neurogenetics from the Max Planck Institute of Experimental Medicine, we generated cKO mice lacking $P / p 1$ in frontal brain structures by crossing $P \not p^{f l / f l}$ mice to mice expressing Cre recombinase under control of the Emx1 promotor (Gorski et al., 2002). By application of immunohistochemical measures PD Dr. Werner and co-workers confirmed the functionality of the cKO mouse model, since Plp was essentially lacking in frontal brain structures such as the PFC and the hippocampal fimbria as well as the CC, but present in control regions. Additionally, Plp and DM20 were absent in the PFC, while expression was not affected in the cerebellum by immunoblot. 


\section{Project II}

\section{(II) Assessment of neuropathology in cKO mice}

To assess neuropathology in cross-sectioned brain slices, we conducted respective immunohistochemical experiments in close collaboration with PD Dr. Werner and co-workers, targeting axonal spheroids $\left(\mathrm{APP}^{+}\right)$, neuroinflammation $\left(\mathrm{IBA}^{+}, \mathrm{MAC}^{+}\right)$and astrogliosis $\left(\mathrm{GFAP}^{+}\right)$. Analysis of these features was performed in sections of the hippocampal fimbria, the CC and the PFC with diverse results. Analysis of the former two brain regions revealed neuropathological features known from other P/p1 KO mouse models (Griffiths et al., 1998; Lüders et al., 2019; Lüders et al., 2017), comprising significant increases in all measured parameters. However, none of these were affected in the PFC.

\section{(III) Behavioral characterization of the mouse model}

Founded on our knowledge that constitutive Php1 KO mice exhibit catatonic signs and executive dysfunction upon adulthood (Gould et al., 2018; Janova et al., 2018), I investigated the relevance of white matter integrity of frontal brain structures in this respect by application of these novel cKO mice. To this end, I conducted a longitudinal and elaborate behavioral characterization of mice of both genders. Experimental approaches covered a wide spectrum of behavioral domains including motor function and coordination, sensory functions, sensorimotor gating, social behavior, depression-like behavior and cognitive abilities such as working memory, spatial memory, cognitive flexibility and perserveration. Interestingly, cKO mice of both genders were not affected in any of these measures. However, cKO mice specifically exhibited an agedependent phenotype comprising catatonic signs and executive dysfunction. The latter was assessed by application of an adapted version of the Puzzle box (Ben Abdallah et al., 2011; Gould et al., 2018; O'Connor et al., 2014) and a modified version of a novel behavioral experiment we recently developed in our group, the Hurdle test. We had previously reported the latter to correlate with catatonic signs in another mouse model of catatonia (Garcia-Agudo et al., 2019). Importantly, the most prominent behavioral phenotype of constitutive $\mathrm{KO}$ mice, impaired RotaRod performance (Griffiths et al., 1998), was unaffected in our novel mouse model measured at adulthood and progressed age as well as via overnight complex running wheel (CRW) performance, thereby delineating frontal brain executive control from this motor phenotype. 


\title{
Original manuscript for publication
}

\author{
Arinrad, S., Siems, S.B., Eichel, M.A., Depp, C., Ronnenberg, A., Hammerschmidt, K, Lüders, \\ K.A., Werner, H.B., Ehrenreich, H. and Nave, K.A. (currently under revision). Isolated \\ catatonia-executive dysfunction complex in aged mice induced by forebrain-specific loss of \\ myelin integrity.
}

Personal contributions: Under supervision of Prof. Dr. Dr. Hannelore Ehrenreich and in close collaboration with PD Dr. Hauke B. Werner and Prof. Klaus-Armin Nave, I was particularly responsible for all behavioral mouse studies conducted in the process of this publication. All mice described here in the context of behavioral assessments were under my care and monitored maintenance, starting at the age of 6 weeks up to the age of 22 to 23 months. During this timeframe, I successively characterized cKO and control mice of both genders behaviorally, by applying elaborate longitudinal behavioral assays along with respective data analyses. In this process, I covered various behavioral domains such as general health monitoring, motor performance and coordination, sensory function, sensorimotor gating, pain perception, social behavior, depression-like behavior and cognitive abilities (supplementary Table 1). In particular, I applied behavioral measures to assess catatonia and executive function in all mice from adolescence to progressed age (Figures $3-4$, supplementary Table 1). In this context, I modified a novel behavioral assay to assess executive function, the Hurdle test (Figure 3 E-F), and further established a modified version of an additional known behavioral test in this regard, the Puzzle box (Figure 3 C-D, Figure 3 - supplement 1). As a critical behavioral control experiment, with respect to known phenotypes upon null mutations of P/p1, I specifically conducted the RotaRod test (Figure 3 - supplement 2) and overnight CRW (Figure 4), to asses motor performance and motor learning abilities. Anja Ronnenberg assisted with distinct behavioral setups and I assisted Dr. Kurt Hammerschmidt in executing experiments in male mice to assess vocalization (supplementary Table 1). I carried out statistical analyses of all behavioral experiments. In conclusion, I was also actively involved in the assembly of figures, display items and supplementary information as well as preparation of the manuscript itself. Specifically, I prepared the supplementary Table 1 and wrote the entire material and methods section on mouse behavior as well as proofreading of the manuscript. 


\title{
Project II
}

\section{Isolated catatonia-executive dysfunction complex in aged mice induced by forebrain-specific loss of myelin integrity}

\author{
Sahab Arinrad ${ }^{1}$, Sophie B. Siems ${ }^{2}$, Maria A. Eichel ${ }^{2}$, Constanze Depp ${ }^{2}$, Anja Ronnenberg ${ }^{1}$, \\ Kurt Hammerschmidt ${ }^{3}$, Katja A. Lüders ${ }^{2}$, Hauke B. Werner, ${ }^{2, *}$ \\ Hannelore Ehrenreich, ${ }^{1, *}$ and Klaus-Armin $\mathrm{Nave}^{2, *}$
}

${ }^{1}$ Clinical Neuroscience, Max Planck Institute of Experimental Medicine, D-37075 Göttingen, Germany

${ }^{2}$ Department of Neurogenetics, Max Planck Institute of Experimental Medicine, D-37075 Göttingen, Germany

${ }^{3}$ Cognitive Ethology, German Primate Center, D-37077 Göttingen, Germany

\section{* Correspondence:}

Dr. Hannelore Ehrenreich

E-mail: ehrenreich@em.mpg.de

Dr. Klaus-Armin Nave

E-mail: nave@em.mpg.de

Max Planck Institute of Experimental Medicine

Hermann-Rein-Str. 3

D-37075 Göttingen, Germany

Tel.: + 49 (551) 3899-757

Fax.: +49 (551) 3899-758

Running title: Forebrain-specific loss of myelin integrity 


\section{Project II}

Key words: Oligodendrocyte, myelin, glia-axonal support, axonopathy, gliosis, proteolipid protein (PLP), hereditary spastic paraplegia (SPG2), executive function, catatonia

Word count: Abstract 192 words; introduction/results/discussion 2878 words; methods 4047 words.

Display items: 4 main figures, 3 supplemental figures, 1 supplemental table.

\section{Author contributions}

S.A., A.R. and K.H. performed behavioral analyses (supervised by H.E.). S.B.S. and M.E. performed neuropathology and K.A.L. immunoblot analysis (supervised by H.B.W.). C.D. performed immunohistochemistry (supervised by K.A.N.). H.B.W., H.E. and K.A.N. designed experiments, interpreted data, and wrote the manuscript. 


\section{Project II}

\section{ABSTRACT}

A key feature of advanced brain aging includes structural defects of intracortical myelin that is associated with secondary neuroinflammation. A similar pathology is seen in specific myelin mutant mice that model 'advanced brain aging' and exhibit a range of behavioral abnormalities. However, the cognitive assessment of these mutants is problematic because myelin-dependent motor-sensory functions are required for quantitative behavioral readouts. To better understand the role of cortical myelin integrity for higher brain functions, we generated mice lacking P/p1, encoding the major integral myelin membrane protein, selectively from ventricular zone stem cells of the mouse forebrain. In contrast to conventional $P / p 1$ null mutants, subtle myelin defects were restricted to the cortex and underlying callosal tracts. Moreover, forebrain-specific P/p1 mutants exhibited no defect of basic motor-sensory performance at any age tested. Surprisingly, several behavioral alterations reported for conventional $P \not p 1$ null mice (Gould et al., 2018) were absent and even social interactions appeared normal. However, with novel behavioral paradigms, we determined catatonia-like symptoms and isolated executive function defects in both genders at older age. This suggest that loss of myelin integrity has an impact on cortical connectivity and underlies specific defects of executive function, emerging only with increasing age. These observations are likewise relevant for human brain aging and neuropsychiatric conditions. 


\section{INTRODUCTION}

In the central nervous system (CNS), oligodendrocytes synthesize myelin to facilitate rapid impulse conduction of axons and to support functional integrity (Nave \& Werner, 2014; Stassart et al., 2018). The formation and preservation of healthy myelin is a prerequisite for normal motor and sensory functions, as indicated by human myelin diseases and their corresponding mouse models, which have been studied in more detail at the molecular and cellular level. The servere developmental defects myelination that affect children at a young age are dominated by the lack of motor development and severe neurological defects, often leading to premature death. Only milder perturbations of myelination make the associated delays of cognitive development obvious, but they are still masked by severe neurological impairments. Some demyelinating diseases of adult onset are degenerative in nature and begin with psychiatric symptoms, including psychosis, such as in metachromatic leukodystrophy (Baumann et al., 2002). Indeed, there is increasing evidence of oligodendrocyte and white matter defects in a range of neuropsychiatric diseases (Nave \& Ehrenreich, 2014; Zhou et al., 2021). Moreover, advanced brain aging in healthy individuals is associated with subtle myelin defects, as first demonstrated by electron microscopy in aged non-human primates (Peters et al., 2002). This raises the question whether structural myelin abnormalities play a role in age-dependent cognitive decline.

In psychiatric patients it is difficult to decide whether the correlation of MRI detectable white matter abnormalities or the ultrastructural loss of myelin integrity are the cause or effect of (age-dependent) neuronal dysfunctions, or even caused by long-term pharmacological interventions (Nave \& Ehrenreich, 2014). Thus, novel genetic animal models are required to define the role of oligodendrocytes and subtle myelin defects as an underlying cause of a psychiatric phenotype (Hagemeyer et al., 2012). Unfortunately, the cognitive testing of mice depends on quantitative behavioral readouts which are affected by subcortical and myelindependent motor-sensory functions.

An entire range of myelin abnormalities with a corresponding wide spectrum of clinical defects characterizes mutations of the proteolipid protein gene (PLP1/Plp1). The X-linked gene is highly expressed in oligodendrocytes and encodes a tetraspan membrane protein of CNS myelin (PLP) along with a minor splice isoform, termed DM20 (Jahn et al., 2009; Milner et al., 1985; Nave et al., 1987). At one end of the disease spectrum, PLP1 mutations cause the expression of misfolded proteins that trigger oligodendroglial ER stress, apoptosis, hypomyelination and 


\section{Project II}

connatal leukodystrophy (Pelizaeus-Merzbacher disease, PMD) with premature death in children and corresponding mouse mutants (Nave and Boespflug-Tanguy, 1996). At the other end, the mere loss-of-function (null) mutations of PLP1, such as by a genomic deletion or in Plp1 null mice (Klugmann et al., 1997), cause late onset neurodegeneration (Griffiths et al., 1998), modeling spastic paraplegia type 2 (SPG2) in humans (Inoue, 2019; Saugier-Veber et al., 1994). The isolated loss of axonal integrity in the presence of myelin led to the concept that oligodendrocytes not only facilitate impulse propagation but also maintain the axonal integrity (Griffiths et al., 1998; Nave, 2010). The latter includes glycolytic support of axonal energy metabolism (Fünfschilling et al., 2012; Lee et al., 2012) and most likely other cellular interactions, such as anti-oxidant defense (Mukherjee et al., 2020).

Loss of myelin integrity and axonal degeneration are invariably associated with secondary neuroinflammation (Kassmann et al., 2007; Lappe-Siefke et al., 2003). Recently, we discovered in different myelin mutant mice that microgliosis causes a unique behavioral phenotype: when mice were placed with their forepaws onto a horizontal bar, wildtype animals turn away within $0.2 \mathrm{~s}$, whereas these mutants exhibit longer response times. We hypothesized that this delay reflects the loss of higher brain (executive) functions rather than a motor impairment. Strikingly, these 'catatonic signs' were completely lost following the targeted pharmacological depletion of microglia (Janova et al., 2018). In an independent study (Gould et al., 2018), Plp1 null mice showed defects in the 'puzzle box' paradigm, which measures the escape latency from a brightly lit open space into a shelter via a mechanically blocked entry. Successfully overcoming these obstacles is also considered an 'executive function' (Ben Abdallah et al., 2011; Gould et al., 2018; O'Connor et al., 2014; Pease-Raissi et al., 2018).

Human catatonia and the loss of executive functions may result from defects in the frontal lobes and its underlying white matter (Arora et al., 2007; Northoff et al., 2004), but experimental evidence is lacking. To test whether executive functions are specifically dependent onforebrain integrity, we targeted the floxed Plp1 allele (Lüders et al., 2019) in mice expressing Cre under control of the Emx1 promoter in ventricular zone stem cells (Gorski et al., 2002). When applying a large battery of behavioral tests on adult mutants of both sexes, we found impaired executive functions at adult ages, coinciding with well-known histopathological signs of advanced white matter aging. Surprisingly, this was in the presence of unchanged motor-sensory performance, memory functions and social behavior. Our genetic data are thus in strong support 
of the hypothesis that the integrity of myelinated fibers in the forebrain is critical for isolated aspects of executive functions.

\section{RESULTS}

\section{Targeting PLP expression in the mouse forebrain}

We crossbred mice harboring a floxed Plp1 allele (Lüders et al., 2017) with mice expressing Cre recombinase under control of the Emx1 promoter (EmxCre mice; Gorski et al., 2002), yielding experimental male $\left(P l p^{f b x / Y *} E m x^{C r y}\right)$ and female $\left(P l p^{f l o x / f b x *} E m x^{C r y}\right)$ mice, termed cKO in the following, as well as male $P / p^{f b x / Y}$ and female Plp ${ }^{f l o x / f b x}$ mice as controls (Ctrl). EMX1 is a homeobox transcription factor in ventricular zone stem cells of the forebrain, including the prefrontal cortex, neocortex, corpus callosum and hippocampal fimbria (Gorski et al., 2002). Substantial reduction of PLP in the fully myelinated target region was confirmed in 22 months old mice by light-sheet microscopy (Figure 1A,B). By Western blotting of prefrontal cortical lysates, PLP/DM20 was hardly detectable, whereas the abundance of myelin basic protein (MBP), another myelin-specific structural protein (Boggs, 2006; Nawaz et al., 2013), was unchanged (Figure 1C). Using the cerebellum as a control region that lacks Emx-Cre expression, the abundance of PLP/DM20 did not differ between genotypes (Figure 1D). The detection of isolated PLP positive cells in the forebrain indicates that recombination efficacy did not reach 100\%. However, since expression of Plp1 is restricted to oligodendrocytes (Lüders et al., 2017; Trapp et al., 1997), phenotypical effects are caused by the vast majority of PLPdeficient oligodendrocytes.

\section{Restricted neuropathology}

Conventional Plp1 null mutant mice develop a secondary axonopathy throughout the CNS at higher age (Griffiths et al., 1998). In Plp1 cKO mice this neuropathology was largely restricted to the forebrain. We studied 17 months and 22 months old mice by immunohistochemistry and found amyloid precursor protein (APP) positive axonal spheroids in white matter structures of the forebrain, such as the hippocampal fimbria (Figure 2A,B and I,J, respectively). Ventricular enlargement was obvious. $\mathrm{APP}^{+}$axonal spheroids were also increased in the dorsal corpus callosum, although less pronounced (Figure 2-supplement $\mathbf{1 A , B}$ ). In contrast, the prefrontal cortex itself did not exhibit $\mathrm{APP}^{+}$axonal spheroids (Figure 2-supplement 1I,J) at 22 months, the oldest age tested. However, axonal pathology included some white matter structures outside the Emx1 expression domain. We presume these reflect distal axonal 


\section{Project II}

projections from neurons in the target region, such as fibers in the internal capsule (Figure 2I,J).

\section{Inflammation in white matter tracts}

At higher age, Plp1 null mutant mice show widespread microglia activation and astrogliosis (de Monasterio-Schrader et al., 2013; Griffiths et al., 1998), presumbably triggered by progressive axonopathy and/or oligodendroglial stress signals. Staining 22 months old mice for astroglial GFAP (glial fibrillary acidic protein) and two microglial markers, MAC3 (lysosomal associated membrane protein 2) and IBA1 (ionized calcium-binding adaptor molecule 1), gliosis was increased in the hippocampal fimbria and in the corpus callosum of Plp1 cKO mice (Figure 2C-H; Figure 2-supplement $\mathbf{1 C}-\mathbf{H}$ ). Interestingly, the prefrontal cortex itself, comprising numerous myelinated axons, did not show these signs of gliosis (Figure 2-supplement 1K-P).

\section{Behavioral defects of Plp1 cKO mice}

In contrast to Plp1 null mice (Griffiths et al., 1998), Plp1 forebrain-specific mutants remained free of spasticity and motor impairments at any age. On the rotarod, the latency to fall did not differ between cKO and control at 6 months and 17 months of age, also when quantified separately for males and females (Figure 3-supplement 2). Details are summarized in supplemental Table 1. Interestingly, in several other behavioral paradigms, in which conventional Plp1 null mice were affected (Gould et al., 2018; Petit et al., 2014), the Plp1 cKO mice were normal; e.g. hot plate (pain), beam balance (coordination), motor performance and olfaction.

On the other hand, $P / p 1$ cKO mice showed and shared defects in paradigms that measured executive functions. Exclusively in our test set-up, the 'bar test' was included and repeatedly performed. For this, mice were placed with their forelimbs against a horizontally held rod (Hagemeyer et al., 2012; Janova et al., 2018; Poggi et al., 2016). Here, adult Plp cKO mice showed an extended response time, in which the apparent immobility constitutes a 'catatonialike' feature (Figure 3A,B). Both female and male groups showed delayed response times on the bar, also when tested at several ages.

We also employed the 'puzzle box' paradigm, a problem solving task, using skills obtained in previous stages of the test (Ben Abdallah et al., 2011; Gould et al., 2018; O'Connor et al., 2014). Placed into the brightly lit area of an open arena, the latency to reach entry into a 'safe box' was monitored, but made increasingly difficult by a series of camouflaging tools. When 
assessed at the age of 18 and 20 months, both male and female Plp1 cKO mice, exhibited significant delays compared to controls, respectively (Figures 3C and 3D).

We additionally found performance differences of Plp1 cKO mice in comparison to controls in an adapted 'hurdle test' that measures 'executive function'. Mice were placed into the middle 7 of a $120 \mathrm{~cm}$ round open field that was divided into squares $(5 \times 5 \mathrm{~cm})$, separated from each other by $5 \mathrm{~cm}$ 'hurdles', but $2.3 \mathrm{~cm}$ higher than in our previous experiments (Garcia-Agudo et al., 2019). We measured the time it took Plp1 cKO mice and controls to reach the periphery of this arena. Since motor performance was not affected, a longer than normal latency reflects a reduced goal-directed orientation towards the periphery, an executive psychomotor function, together perhaps with dampened motivational force. While behaving normally at age 6 months, when analyzed at the age of 18 months, male Plp1 cKO mice required more time to reach the periphery in two of two trials (Figure 3F), in agreement with an age-dependent decline of white matter integrity. Interestingly, in subsequent trials mutant mice often lost this abnormal delay, which confirms an abnormal executive function rather than motor impairments. Female Plp1 cKO mice displayed the longer delay in the first of two trials already at the age of 6 months, but when tested again at 20 months only with a non-significant trend. This demonstrates contributory effects of both, sex and age, in this paradigm (Figure 3E).

Finally, we assessed the performance of Plp1 cKO and controls at more advanced age (23 months) on the 'complex running wheel' (CRW), a paradigm testing cortical motor learning that involves myelinating oligodendrocytes (Gibson et al., 2014; McKenzie et al., 2014). Both female and male groups displayed significantly reduced total running distance and running times (Figures 4A,B and 4C,D, respectively). Since the average running speed did not differ between cKO and control groups (Figure 4E,F), this performance difference may reflect a reduced drive rather than altered motor learning, in accordance with a feature of executive dysfunction in the Puzzle box and Hurdle test paradigms. 


\section{Project II}

\section{DISCUSSION}

We report the generation and behavioral analysis of a novel mouse mutant, in which the lack of oligodendroglial Plp1 expression was spatially restricted to neocortex, hippocampus and corpus callosum by targeting Plp1 in ventricular zone stem cells of the embryonic forebrain. Since Plp1 is only required by oligodendrocytes in the CNS (Lüders et al., 2019), the resulting conditional mouse exhibits a region- and cell type-specific defect. This allowed behavioral tests of higher brain functions that rely on motor output, because the myelinating oligodendrocytes in cerebellum and spinal cord, which contribute to basic motor-sensory functions, were spared.

The Plp1 gene was chosen as a target, because the cardinal features of PLP-deficiency are slowly-progressive axonopathy in the presence of close to normal amounts of myelin, with ultrastructural features reminiscent of advanced brain aging (Griffiths et al., 1998; Janova et al., 2018). Moreover, the Plp1 null mutation in mice is a genetic model of human Spastic Paraplegia type-2 (SPG2; Garbern et al., 2002). The conventional Plp1 null mutation in mice (Klugmann et al., 1997), which has recently been studied at the behavioral level (Gould et al., 2018), can be directly compared.

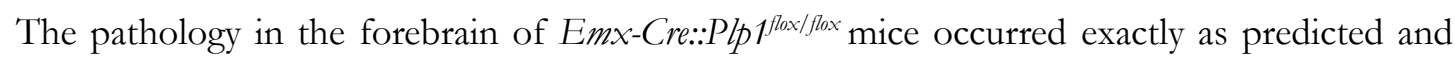
includes previously described subtle ultrastructural abnormalities of PLP-deficient myelin (Klugmann et al., 1997; Möbius et al., 2016; Patzig et al., 2016; Rosenbluth et al., 2006) that become disease relevant at age one year. We have shown that PLP, which provides structural stability to compact myelin, also stabilizes the architecture of the 'myelinic channel system' (Steyer et al., 2020). The latter is a cytosolic space that connects the oligodendroglial cell body with the peri-axonal myelin compartment that contains transporters involved in axonal metabolic support. We could further show that conventional Plp1 KO mice, which are well myelinated, exhibit lower ATP levels in axons of the optic nerves compared to controls, when analyzed with transgenically expressed metabolic sensors (Trevisiol et al., 2020). This makes it likely that conduction blocks, axonal swellings and neurodegeneration are caused by perturbed axonal energy metabolism (Fünfschilling et al., 2012; Lee et al., 2012; Saab et al., 2016; Trevisiol et al., 2017). In addition, axonal degeneration leads to secondary neuroinflammation. We note that other myelin mutants, such as Cnp KO mice (Lappe-Siefke et al., 2003), exhibit an even earlier onset of axonal pathology that virtually coincides with the onset of neuroinflammation (Edgar et al., 2009). 


\section{Project II}

This raises the possibility that inflammation in myelinated tracts is a more general response of resident microglia to oligodendrocyte dysfunctions, possibly reflecting the failure to deliver lactate to the axonal compartment and its release into the extracellular space. It is this secondary neuroinflammation that contributes to reversible catatonic signs in myelin mutant mice (Janova et al., 2018) and that can now be defined as a clinical feature of forebrain-specific white matter disturbance.

Theoretically, the specific behavioral defects of Plp1 cKO mice could be caused by (1) the moderate decrease of the axonal conduction velocity that was previously reported (Gould et al., 2018; Gutiérrez et al., 1995), (2) the progressive axonopathy that is caused by energy deficits and is likely preceded by conduction blocks in fiber tracts that appear morphologically intact (Trevisiol et al., 2020), (3) the secondary neuroinflammation and gliosis, as also documented for Cnp KO mice (Garcia-Agudo et al., 2019; Janova et al., 2018), another mutant with structural abnormalities of myelin (Lappe-Siefke et al., 2003; Snaidero et al., 2017), or (4) any combination hereof. The age-dependent increase of symptom severity suggests that the responsible mechanisms are the progressive myelin-dependent axonopathy (including conduction blocks) plus neuroinflammation rather than conduction delays. That is in agreement with the "rescue" of the catatonic signs, which we consider a readout of reduced executive functions, by microglial depletion (Janova et al., 2018). We also note that reduced conduction velocity has by itself little impact on cortical processing, as we have shown for auditory signalsin shiverer mice (Moore et al., 2019).

With respect to CNS regions, the present work further uncouples the role of myelinated tracts for basic motor performance and executive functions. Conventional Plp1 KO mice exhibit first behavioral abnormalities at the age of 3 months (Gould et al., 2018), i.e. before the impairment of motor performance that we had initially determined by rotarod experiments (Griffiths et al., 1998). Here, we show in Plp1 cKO mice that the forebrain-specific loss of axon-myelin integrity impairs selectively executive functions that require the multimodal integration of cortical processing upstream of any motor output. Motor performance itself was spared at any age tested. Also olfactory and thalamic input as well as emotionally relevant input into (and from) the amygdala should be less affected in Plp1 cKO mice, which could explain different behaviors of conventional Plp1 KO mice (Gould et al., 2018).

The principle finding that (PLP-dependent) aging processes affecting myelin and axonal integrity in the forebrain cause the impairment of executive functions is relevant to higher brain 


\section{Project II}

functions in aging humans independent of PLP itself. Similar ultrastructural abnormalities of myelin were found in the cortex of aged primates (Peters et al., 2012) and are associated with low grade inflammation, all of which is triggered in our mouse model prematurely by the absence of PLP. We have previously shown that mice with reduced Cnp1 gene dosage show virtually all these features of the aging white matter approximately 8 months earlier than corresponding wildtype controls, and are thus models of "advanced" aging (Hagemeyer et al., 2012). It is plausible that our observations in aging white matter and its functional impact in mouse mutants can be carefully extrapolated to the aging human brain, which is not amenable to comparable ultrastructural analyses. The resulting working hypothesis is that age-dependent loss of structural integrity of myelinated fibers in the forebrain reduces executive functions. It will be important to learn whether these changes are a risk factor for age-dependent psychiatric conditions, including dementia. 


\section{MATERIALS AND METHODS}

\section{Mouse model}

To delete the $P \not p 1$ gene in the forebrain we interbred $P / p^{f o x}$ mice in which exon 3 of the $P / p 1$ gene is flanked by loxP sites (Lüders et al., 2019; Lüders et al., 2017; Wang et al., 2017) with mice expressing Cre recombinase under control of the Emx1 gene promoter (Gorski et al., 2002) on C57Bl/6N background. Genotyping was as reported previously (Gorski et al., 2002; Lüders et al., 2017). Experimental male $P / p^{f b x} / Y^{*} E m x^{C r e}$ and female $P / p^{f l o x} / f b x * E m x^{C r e}$ mice are termed conditional knockout mice $(\mathrm{cKO})$ whereas male $P / p^{f b x} / Y$ and female $P / p^{f l o x / f b o x}$ mice served as controls (Ctrl). Mice were bred and kept in the animal facility of the Max Planck Institute of Experimental Medicine with a $12 \mathrm{~h}$ light/dark cycle and 2-5 mice per cage. All experiments were performed in accordance with the German animal protection law (TierSchG) and approved by the Niedersächsisches Landesamt für Verbraucherschutz und Lebensmittelsicherheit (LAVES); License numbers were 33.19-42502-04-15/1833 and 33.1942502-04-18/2803.

\section{Light-sheet microscopy (LSM)}

Light-sheet microscopy to detect PLP labelling displayed in Figure 1 A,B was perfomed as follows. Animals were sacrificed using CO2 followed by transcardial perfusion with HBSS and Paraformaldehyd (4\%) in PBS. Brains were extracted and postfixated in 4\% PFA/PBS overnight and stored in PBS at $4^{\circ} \mathrm{C}$ until further use. Brains were cut using a $1 \mathrm{~mm}$ custom made brain matrix and subjected to whole mount staining and clearing. Samples were dehydrated in a methanol/PBS series $(50 \%, 80 \%, 100 \%)$ followed by overnight bleaching and permeabilization in a mixture of 5\% $\mathrm{H} 2 \mathrm{O} 2 / 20 \%$ dimethyl sulfoxide (DMSO) in methanol at $4^{\circ} \mathrm{C}$. Samples washed further in methanol prior to incubation in $20 \% \mathrm{DMSO}$ in methanol at RT for $2 \mathrm{~h}$. Samples were then rehydrated using a descending methanol/PBS series $(80 \%, 50 \%$, PBS)and further washed with in PBS/0.2\% TritonX-100 for $2 \mathrm{~h}$. The samples were then incubated overnight in $0.2 \%$ TritonX-100, 20\% DMSO, and $0.3 \mathrm{M}$ glycine in PBS at $37^{\circ} \mathrm{C}$ and blocked using PBS containing 6\% goat serum, 10\% DMSO and $0.2 \%$ Triton-X100 for 2 days at $37^{\circ} \mathrm{C}$. Samples were retrieved, washed twice in PBS containing $0.2 \%$ Tween20 and $10 \mu \mathrm{g} / \mathrm{ml}$ heparin $(\mathrm{PTwH})$ at RT for $1 \mathrm{~h}$ and incubated with primary antibody solution [rat anti-PLP (aa3, 1:250), mouse anti-CNP (Atlas, 1:250)] for 14 days at $37^{\circ} \mathrm{C}$. After several washes in PTwH, samples were incubated with secondary antibody solution (goat anti-rat Alexa555; goat antimouseAlexa633) for 7 days at $37^{\circ} \mathrm{C}$. Prior to clearing, the samples were again washed in PTwH and brain slices were embedded in $2 \%$ Phytagel in PBS. Slices were dehydrated using an 


\section{Project II}

ascending series of Methanol/PBS (20\%, 40\%, 60\%, 80\%, 2x 100\% 1h, RT) followed by overnight incubation in a mixture of 33\% dichloromethan (DCM) and 66\% methanol at RT. Samples were further delipidated by incubation in 100\% DCM for 40min and transferred to pure ethyl cinnamate (Eci; Sigma Aldrich \#112372) as clearing agent. LSM was performed using a LaVision Ultramicroscope II equipped with a 2x objective, corrected dipping cap and zoom body. Slices embedded in phytagel tubes were mounted onto the sample holder. Images were acquired in mosaic acquisition mode with the following specifications: $5 \mu \mathrm{m}$ sheet thickness; $30 \%$ sheet width; $2 \mathrm{x}$ zoom; $2 \mathrm{x} 3$ tiling; $4 \mu \mathrm{m}$ z-step size; dual site sheet illumination; $100 \mathrm{~ms}$ camera exposure time. Green, red and far red fluorescence were recorded using $488 \mathrm{~nm}$, $561 \mathrm{~nm}$ and 633 laser excitation (30\%) and respective emission filters. Images were loaded into Vision4D 3.3 (Arivis) and stitched using the tile sorter setup. Datasets were pseudocoloured and visualized in 3D (maximum intensity mode).

\section{Immunohistochemistry}

Immunohistochemistry for neuropathological analysis displayed in Figure 2 and Figure 2supplement 1 was performed as previously described (Lüders et al., 2019; Patzig et al., 2016). Antibodies were specific for MAC3 (1:400; Pharmingen 553322; clone M3/84), IBA1 (1:1,000; Wako 019-19741), APP (1:1,000; Millipore MAB 348) or GFAP (1:200; Novocastra NCLGFAP-GA5). Images were captured at 20x (GFAP, IBA1, MAC3) or 40x (APP) magnification using a bright-field light microscope (Zeiss AxioIm7ager Z1) coupled to a Zeiss AxioCam MRc camera controlled by Zeiss ZEN 1.0 software and processed using Fiji. To quantify axonal swellings, the region of interest was selected and APP-immunopositive axonal swellings were counted. To quantify brain region immunopositive for IBA1, MAC3 or GFAP, the region of interest was selected and analyzed using an ImageJ plug-in (de Monasterio-Schrader et al., 2013; Lüders et al., 2019; Lüders et al., 2017) for semi-automated analysis. Per genotype (Ctrl, cKO), 5-7 male mice were assessed at the age of 22 months as indicated by the data points in Figure 2 and Figure 2-supplement 1, in which n numbers represent individual mice. Assessment of neuropathology was performed blinded to the genotype. Statistical analysis was performed by two-tailed unpaired t-test in GraphPad Prism 6.0. Levels of significance were set as $\mathrm{p} 0<0.05$ $(*)$, p $0<0.01(* *)$, and p $0<0.001(* * *)$.

\section{Immunoblotting}

Prefrontal cortex (frontal to bregma 5.22) and cerebellum were dissected from mice and homogenized in 1x TBS with protease inhibitor (Complete Mini, Roche). Protein concentration was measured using the DC protein assay (BioRad). Immunoblotting displayed in Figure 1C,D 
was essentially as described (Kusch et al., 2017; Schardt et al., 2009). Briefly, lysates from prefrontal cortex $(3.2 \mu \mathrm{g}$ for PLP/DM20 and $1 \mu \mathrm{g}$ for actin) and cerebellum (0.8 $\mu \mathrm{g}$ for PLP/DM20 and $1 \mu \mathrm{g}$ for actin) were separated on 15\% SDS-polyacrylamide gels and blotted onto PVDF membranes (Hybond, Amersham) using the Novex Semi-Dry Blotter (Invitrogen). Primary antibodies were incubated in $5 \%$ milk powder in TBST over night at $4^{\circ} \mathrm{C}$. Primary antibodies were specific for PLP/DM20 (A431; 1:5000; (Jung et al., 1996)), MBP (1:500; DAKO) and actin (1:1000 for cerebellum, 1:5000 for prefrontal cortex; Sigma). Secondary HRPcoupled antibodies (dianova) were detected using the ChemoCam system (Intas).

\section{Mouse maintenance and behavioral tests}

All mice for behavioral tests were maintained in ventilated cabinets (Scantainers, Scanbur, Karlslunde, Denmark), separated by gender, under standard laboratory conditions, including a $12 \mathrm{~h}$ light/dark cycle (lights off at $7 \mathrm{PM}$ ) at $20-22^{\circ} \mathrm{C}, 50-60 \%$ humidity and with access to food and water ad libitum. Upon weaning, mice were separated by gender and genotype and remained group-housed in standard plastic cages (2-5 mice per cage). Male mice were singlehoused at age of 6 months, due to requirements of experimental tests. Male and female cKO mice $(\mathrm{N}=14$ and 20, respectively) were compared to respective control mice $(\mathrm{N}=16$ and 17 , respectively) in all behavioral experiments. A series of behavioral paradigms, described below, was conducted on mice throughout lifespan, covering ages between 2 - 23 months (supplemental Table 1). General health status of mice was continuously monitored by body weight, home-cage observation including general activity and appearance, nest building and interaction with littermates. Group sizes decreased upon progressing age due to natural death. In all behavioral experiments, mice were allowed to habituate to conditions of the experimental room for a minimum of 30 min prior to testing. Male and female mice were tested separately.

\section{Muscle strength, Motor coordination and Motor Learning}

Rotarod - motor coordination and learning. The rotarod (ENV-577M, Med Associates Inc. Georgia, Vermont, USA) was performed as previously described (Dere et al., 2014). Briefly, all mice were tested in a total of two trials over two consecutive days. Each trial consisted of a maximum of $5 \mathrm{~min}$, in which mice had to run continuously on a horizontal rotating drum accelerating from 4-40 rpm. The latency to fall (s) was assessed for each mouse during both trials. Mice of both genders were tested in the rotarod at the age of 6 and 17 months.

Grip-strength - forelimb muscle strength. The forelimb grip strength of male and female mice was assessed at age 6,13 and 15 months. The test was performed as previously described (Netrakanti et al., 2015). Briefly, each mouse was tested in a total of three 


\section{Project II}

consecutive trials. During each trial, mice were lifted gently by their tails and allowed to grasp a wire of the grip strength meter (TSE Systems, Bad Homburg, Germany). Upon grasping the wire, mice were brought into a horizontal position before being gently pulled back by their tails, to assess the applied forelimb force. The average grip strength per mouse was calculated.

Beam balance - motor coordination. Motor coordination was assessed at age 13 months, using beam balance test as previously described (Netrakanti et al., 2015). Briefly, the ability of mice to cross elevated beams (59 cm length) of decreasing diameter $(25 \mathrm{~mm}, 10 \mathrm{~mm}$ or $8 \mathrm{~mm}$ ) was measured. Mice were placed at the illuminated end of the respective beam and the latency to reach the target zone, an attached cardboard cage with bedding, was recorded. The test was conducted over three consecutive days, starting with the $25 \mathrm{~mm}$ beam (day 1), then $10 \mathrm{~mm}$ (day 2) and $8 \mathrm{~mm}$ beam (day 3). Each mouse was tested in a maximum of three trials per day, in case it fell off the beam, and given $60 \mathrm{~s}$ to reach the target.

\section{Hearing and Sensorimotor Gating}

Pre-pulse inhibition of the acoustic startle response (PPI). Male and female mice were tested for both general hearing and sensorimotor gating at age of 6 and 18 or 6 and 20 months, respectively. A detailed protocol of PPI was reported previously (Netrakanti et al., 2015). Sensorimotor gating experiments were conducted within sound attenuating chambers (TSE Systems, Bad Homburg, Germany), in which acoustic stimuli (120 dB) both with and without a preceding pre-pulse evoked startle responses, recorded by a force-sensitive platform. PPI, using a 70,75 or $80 \mathrm{~dB}$ pre-pulse, was calculated by following formula: \%PPI $=100-$ [(startleamplitude after prepulse) / (startle amplitude after pulse only) x 100] (Pan et al., 2018). To assess hearing abilities of male and female mice, the amplitude of response (arbitrary units) to $65 \mathrm{~dB}$ background noise as well as to six pulse-alone trials with startle stimuli of $120 \mathrm{~dB}$ was measured.

\section{Sensory functions}

Hot plate test - nociception. Pain perception in male and female was assessed at the age of 13, 15, 17 and 20 months, using the hot plate test as described (Dere et al., 2014). Briefly, mice were placed on a preheated $\left(55^{\circ} \mathrm{C}\right)$ metal plate (Ugo Basile Srl, Comerio, Italy) and the latency (s) to retract by jumping or licking of the hind paws was recorded. Mice were exposed for a maximum of $40 \mathrm{~s}$, used as cut-off time, in case they did not show an aversive response to the heated plate.

Cued platform training in the Morris water maze - vision. General vision of male and female mice was evaluated within the Morris water maze experiment at age 19 or 13 months, 
respectively, as detailed before (Dere et al., 2014). Vision was assessed during the first two days of acquisition training, in which an escape platform was submerged $1 \mathrm{~cm}$ below the surface of opaque water. A small blue flag protruding above the water surface was attached at the center of the platform and used as cue for locating the escape platform. Each mouse was tested on two consecutive days á 4 trials per day with a 5 min inter-trial-interval (ITI). Escape latency, velocity of swimming and path length were recorded with video-tracking system (Viewer3, Biobserve GmbH, Bonn, Germany).

\section{Social behavior and social preference}

Ultrasound vocalization - communication. Ultrasonic vocalization was evaluated as described in male mice only, at age 6 months (Dere et al., 2014; Hammerschmidt et al., 2012). Male mice of both genotypes were single-housed one week prior to testing. Each male mouse was exposed to an anesthetized unfamiliar C57Bl/6N WT female within its home-cage for 3 min. Number of ultrasonic calls of the resident males and latency to first call (s) were recorded. Social boxes in the IntelliCage $\mathbb{B}$ design - pheromone-based social preference.

Pheromone-based social preference was evaluated at age 6 months in female mice only as described (Dere et al., 2018). Briefly, mice underwent anesthesia for subcutaneous implantation of ISO standard transponders (PM162-8) below the skin of the neck, one day prior to group-housing within IntelliCages ${ }^{\circledR}$. Mice remained in the cages for a total of 6 days for an IntelliCage ${ }^{\circledR}$-based behavioral phenotyping (see below). Social preference was tested on the last day by connecting two social boxes to the left and right side of the cages. Connection of the boxes was carried out via plastic tubes equipped with 2 ring RFID antennas, which monitored entrance and exit of individual mice to each box. Initially, both boxes were filled with fresh bedding and animals were allowed to freely explore for a $1 \mathrm{~h}$ habituation. Subsequently, used bedding containing pheromones of male $\mathrm{C} 3 \mathrm{H}$ mice was added to one of the boxes and the time spent in the pheromone vs. non-pheromone box was recorded for another hour.

\section{Cognitive flexibility, episodic-like memory and anhedonia}

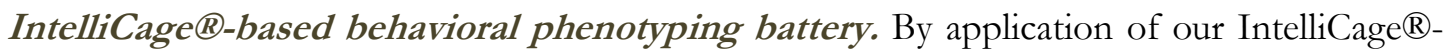
based behavioral phenotyping design, we assessed multiple facets of cognition as well as sucrose preference, as measure of anhedonia, in female mice only, at age 6 months as described (Dere et al., 2018). One day after subcutaneous implantation of transponders (see above), mice were group-housed, separated by genotype, and remained in the IntelliCages ${ }^{\circledR}$ for 6 days. Place learning was acquired within the first $24 \mathrm{~h}$ (day 1), in which an individual mouse learned that only one out of four corners was rewarded with water, while the other 


\section{Project II}

corners remained blocked. The number of mice assigned to each corner was balanced and semi-randomly determined. On day 2, reversal learning was assessed to measure cognitive flexibility as well as perseveration. Mice had to learn that the previously rewarded corner was now blocked, and instead the diametrically opposed corner now rewarded. Sucrose preference was assessed on day 3 by comparing preference for a corner rewarded with a $2 \%$ sucrose solution over another corner rewarded with tap water. During these $24 \mathrm{~h}$, the two previously blocked corners were now rewarded with either sucrose solution or tap water, whereas the remaining corners were blocked. The visits to the respective target corners during place and reversal learning as well as sucrose preference testing were used for statistical analysis. On days 4-5 mice had again access to two rewarding corners providing either a sucrose solution or tap water. However, these corners were now again the diametrically opposed corners to day 3 and access to the corners was only provided for a limited time, namely during the first two hours of the active phase of the mice (6.00-8.00 PM). Hence, mice were required to form a multi-modal association containing the information on the type of reward provided (what) as well as their locations (where) and the time at which to expect the reward (when), rendering this approach an experimental model for the assessment of episodic memory comparably to humans. Visits to rewarded corners during acquisition (day 4) and retrieval (day 5) of episodic-like memory were recorded and the delta between visits to the corner providing sucrose solution and the corner providing tap water was calculated.

\section{Cognitive flexibility, episodic-like memory and anhedonia}

Morris water maze. Spatial memory as well as cognitive flexibility and perseveration, via reversal learning, were evaluated in male and female mice at the age 19 or 13 months, respectively, using the Morris water maze task. The test was conducted as described (Dere et al., 2014; Netrakanti et al., 2015). Mice were tested within a circular tank (diameter $1.2 \mathrm{~m}$ and depth $0.6 \mathrm{~m})$ filled with opaque water $\left(25 \pm 1^{\circ} \mathrm{C}\right)$ in various successive phases, starting with two days of cued platform training as described above (see: vision). Subsequently, the blue flag cue was detached from the escape platform $(10 \mathrm{~cm}$ x $10 \mathrm{~cm})$, which was submerged $1 \mathrm{~cm}$ below the water surface and relocated within the tank, and mice were tested for 8 days during hidden platform training. Throughout these days mice had to form a spatial memory for the escape platform using various extra-maze cues placed on the walls of the testing room. During all training phases mice were tested in 4 daily trials with an ITI of $5 \mathrm{~min}$ and the individual performances were recorded with a video-tracking system (Viewer3, Biobserve GmbH, Bonn, Germany) for subsequent analysis. Training was followed by a probe test to assess spatial memory and the time spent in the target quadrant of the maze as well as visits and the latency 
to the target quadrant were used for statistical analysis. Moreover, total distance swam and the average swimming velocity were analyzed, to exclude potential motor deficits. Finally, mice were exposed to 4 days of reversal training, in which the only difference to the hidden platform training was that the escape platform was relocated into a new quadrant of the tank. Reversal training was followed by another probe trial, wherein the same parameters were determined as in the first probe test.

\section{Working memory}

$Y$ maze continuous alternation. Spontaneous alternation was assessed in male and female mice of 20 months age, in a Y shaped maze (Dere et al., 2014). Mice were individually placed into the center of a triangle-shaped maze with three identical open arms $(7.5 \mathrm{~cm} \mathrm{x} 18 \mathrm{~cm} \mathrm{x}$ $23.5 \mathrm{~cm}$ ) and allowed to freely explore the maze for a total of $5 \mathrm{~min}$. Performance of all mice including total number of arm entries, defined as entering with all four paws, was recorded with a video-tracking system (Viewer3, Biobserve GmbH, Bonn, Germany). An alternation ratio, defined as the number of alternating triplets multiplied by 100 and divided by the total number of entries, was calculated.

\section{Catatonic signs and executive function}

Bar test. Catatonic signs were assessed in male and female mice at various ages ranging from 2-21 months in male and 2-22 months in female mice. The bar test was conducted as described before (Garcia-Agudo et al., 2019; Janova et al., 2018). Briefly, mice were gently carried by the tail and brought into proximity of a horizontal bar made of stainless steel. Upon grasping the bar with both forepaws and standing upright, the tail was released. Mice were tested in two consecutive trials, which were recorded with a high-resolution camcorder (Sony HDR-CX405, Sony, Tokyo, Japan) and the time spent immobile at the bar (s) was determined.

Modified Hurdle test. The hurdle test is a novel tool to measure executive dysfunction in catatonia (Garcia-Agudo et al., 2019). The test setup comprises a circular open field arena containing a polyvinylchloride comb inset (119 cm diameter), made of equally built (10 x 10 $\mathrm{cm}$ ) connected combs, and 140 lux light intensity at the center of the arena, to motivate mice to move to the periphery. We applied minor modifications to the previously reported setup, by increasing the height of the connected combs from $2.7 \mathrm{~cm}$ to now $5.0 \mathrm{~cm}$ and adding fresh woodchip bedding into each comb. These modifications were applied, to increase the challenge, while simultaneously reducing aversive responses to the novel environment upon introduction of bedding as a familiar and comfortable texture. The experiment was conducted in male and female mice at age 6 and 18 or 6 and 20 months, respectively, as described (Garcia- 


\section{Project II}

Agudo et al., 2019). Briefly, mice were placed into the center of the above-mentioned inset and their performance until reaching the periphery or the cut-off time of $5 \mathrm{~min}$, was recorded using the Viewer 3 tracking software (Biobserve GmbH, Bonn, Germany). All mice were tested in two consecutive trials with a 5 min ITI. Executive performance was assessed by calculating the ratio of latency to periphery (s) divided by the number of crossed hurdles (\#). To account for animals that did not overcome any hurdle, we calculated the ratio as $[(\mathrm{s}) /(\#+1)]$.

Puzzle box. As an additional test assessing executive function, the puzzle box was conducted on male and female mice at age 18 or 20 months, respectively. We employed the test as 17 described (O'Connor et al., 2014) with minor modifications. The experimental setup comprises a rectangular-shaped arena $(75 \mathrm{~cm} \times 28 \mathrm{~cm} \times 25 \mathrm{~cm})$ split into an enclosed shelter $(15 \mathrm{~cm} \times 28$ $\mathrm{cm})$ and an illuminated (140 lux) open compartment $(60 \mathrm{~cm} \times 28 \mathrm{~cm})$. The compartments are connected to each other via a small doorway, centered at the front wall of the shelter $(4 \mathrm{~cm}$ width), through which mice can escape into the shelter upon placement into the open compartment. Over a course of 5 consecutive days, mice were required to overcome a total of 5 challenges of increasing difficulty, within a limited amount of time, to reach the shelter. We employed the following challenges: 1. open doorway, 2. gateway within doorway, 3. gateway filled with bedding, 4. plug made of paper tissue, 5. plug made of nesting material (shredded cardboard paper). Each mouse was tested in a total of three trials per day, in which mice were exposed to 2 different challenges daily. During the first trial on each day, mice were exposed to the challenge they had to overcome last on the day before, whereas the following two trials measured escape latencies upon introduction of a novel unfamiliar challenge. Exceptions to this approach were the very first trial on day 1 (open entry), which was tested only this one time, and day 5, since mice were tested only once with the most difficult challenge (plug made of nesting material) on day 5. Cut-off time was increased from 4 to 6 minutes during challenges 4 and 5 , to provide sufficient time for mice to be able to unplug the doorway into the shelter. Performance of each mouse was recorded with video-tracking system (Viewer3, Biobserve GmbH, Bonn, Germany) and averaged escape latency for each challenge measured.

Complex wheel running. Overnight voluntary complex wheel running (CRW) was conducted at age 23 months, as an additional measure of both drive and motor-cognitive performance. Mice of both genotypes and genders were single-housed and exposed to CRW (TSE Systems, Bad Homburg, Germany) for $24 \mathrm{~h}$. Placement of mice into the respective CRW cages was carried out in the morning (8-9 AM) allowing the mice to familiarize themselves with the novel environment throughout the day. CRW are defined by randomly omitted bars, providing a motor-cognitive and coordinatory challenge. Voluntary running on CRW was recorded automatically (Phenomaster software, TSE Systems, Bad Homburg, Germany) yielding 
information on time spent running ( $\mathrm{min})$, total distance run $(\mathrm{cm})$ and running velocity $(\mathrm{cm} / \mathrm{min})$. Analysis of CRW performance was conducted over $12 \mathrm{~h}$ during the active phase (lights off from 7 PM - 7 AM).

\section{Statisical Analyses}

Computation of an appropriate sample size for this study was carried out via G*Power software (Faul et al., Behavior Research Methods 2007, https://doi.org/10.3758/BF03193146) based on the following statistical requirements and assumptions: $\alpha$-error 0.05 , $\beta$-error 0.085 , statistical effect size 1.000, based on previous IntelliCage-based experiments in our lab. We chose the IntelliCage-based assay serving a as a crucial limiting experiment, due to the high cognitive demand of the setup (see also: Dere et al., Neurobiology of learning and memory 2018, doi: 10.1016/j.nlm.2018.02.023.) Mice of both genders (male/female) and both genotypes (control/conditional mutant) were used, with each mouse being a biological replicate in each assay.

Statistical analysis was performed using GraphPad Prism 9.0. Between-group comparisons were made by either one-way or two-way analysis of variance (ANOVA) with repeated measures or t-test for independent samples. Mann Whitney U, Wilcoxon tests were used if the normality assumption was violated (as assessed by the Kolmogorov-Smirnov test). Data presented in the figures and text are expressed as mean \pm SEM; p-values $<0.05$ were considered significant. Statistical outliers were assessed using the GraphPad Grubbs outlier tool, but no such outliers had to be removed from the data. Only mice of any gender or genotype that did not perform in an experiment were excluded from statistical analyses. 


\section{Project II}

\section{REFERENCES}

Arora, M., \& Praharaj, S. K. (2007). Butterfly glioma of corpus callosum presenting as catatonia. The World Journal of Biological Psychiatry, 8(1), 54-55.

Baumann, N., Turpin, J.-C., Lefevre, M., \& Colsch, B. (2002). Motor and psycho-cognitive clinical types in adult metachromatic leukodystrophy: genotype/phenotype relationships? Journal of Physiology-Paris, 96(3-4), 301-306.

Ben Abdallah, N. M.-B., Fuss, J., Trusel, M., Galsworthy, M. J., Bobsin, K., Colacicco, G., et al. (2011). The puzzle box as a simple and efficient behavioral test for exploring impairments of general cognition and executive functions in mouse models of schizophrenia. Experimental neurology, 227(1), 42-52.

Boggs, J. (2006). Myelin basic protein: a multifunctional protein. Cellular and Molecular Life Sciences CMLS, 63(17), 1945-1961.

de Monasterio-Schrader, P., Patzig, J., Möbius, W., Barrette, B., Wagner, T. L., Kusch, K., et al. (2013). Uncoupling of neuroinflammation from axonal degeneration in mice lacking the myelin protein tetraspanin-2. Glia, 61(11), 1832-1847.

Dere, E., Dahm, L., Lu, D., Hammerschmidt, K., Ju, A., Tantra, M., et al. (2014). Heterozygous ambra1 deficiency in mice: a genetic trait with autism-like behavior restricted to the female gender. Frontiers in behavioral neuroscience, 8, 181.

Edgar, J. M., \& Nave, K.-A. (2009). The role of CNS glia in preserving axon function. Current opinion in neurobiology, 19(5), 498-504.

Fünfschilling, U., Supplie, L. M., Mahad, D., Boretius, S., Saab, A. S., Edgar, J., et al. (2012). Glycolytic oligodendrocytes maintain myelin and long-term axonal integrity. Nature, 485(7399), 517-521.

Garbern, J. Y., Yool, D. A., Moore, G. J., Wilds, I. B., Faulk, M. W., Klugmann, M., et al. (2002). Patients lacking the major CNS myelin protein, proteolipid protein 1, develop length-dependent axonal degeneration in the absence of demyelination and inflammation. Brain, 125(3), 551-561.

Garcia-Agudo, L. F., Janova, H., Sendler, L. E., Arinrad, S., Steixner, A. A., Hassouna, I., et al. (2019). Genetically induced brain inflammation by Cnp deletion transiently benefits from microglia depletion. The FASEB Journal, 33(7), 8634-8647.

Gibson, E. M., Purger, D., Mount, C. W., Goldstein, A. K., Lin, G. L., Wood, L. S., et al. (2014). Neuronal activity promotes oligodendrogenesis and adaptive myelination in the mammalian brain. Science, 344(6183), 1252304. 


\section{Project II}

Gorski, J. A., Talley, T., Qiu, M., Puelles, L., Rubenstein, J. L., \& Jones, K. R. (2002). Cortical excitatory neurons and glia, but not GABAergic neurons, are produced in the Emx1-expressing lineage. Journal of Neuroscience, 22(15), 6309-6314.

Gould, E. A., Busquet, N., Shepherd, D., Dietz, R. M., Herson, P. S., de Souza, F. M. S., et al. (2018). Mild myelin disruption elicits early alteration in behavior and proliferation in the subventricular zone. Elife, 7, e34783.

Griffiths, I., Klugmann, M., Anderson, T., Yool, D., Thomson, C., Schwab, M. H., et al. (1998). Axonal swellings and degeneration in mice lacking the major proteolipid of myelin. Science, 280(5369), 1610-1613.

Gutiérrez, R., Boison, D., Heinemann, U., \& Stoffel, W. (1995). Decompaction of CNS myelin leads to a reduction of the conduction velocity of action potentials in optic nerve. Neuroscience letters, 195(2), 93-96.

Hagemeyer, N., Goebbels, S., Papiol, S., Kästner, A., Hofer, S., Begemann, M., et al. (2012). A myelin gene causative of a catatonia-depression syndrome upon aging. EMBO molecular medicine, $4(6), 528-539$.

Hammerschmidt, K., Radyushkin, K., Ehrenreich, H., \& Fischer, J. (2012). The structure and usage of female and male mouse ultrasonic vocalizations reveal only minor differences. PloS one, 7(7), e41133.

Inoue, K. (2019). Pelizaeus-Merzbacher Disease: Molecular and Cellular Pathologies and Associated Phenotypes Myelin (pp. 201-216): Springer.

Jahn, O., Tenzer, S., \& Werner, H. B. (2009). Myelin proteomics: molecular anatomy of an insulating sheath. Molecular neurobiology, 40(1), 55-72.

Janova, H., Arinrad, S., Balmuth, E., Mitjans, M., Hertel, J., Habes, M., et al. (2018). Microglia ablation alleviates myelin-associated catatonic signs in mice. The Journal of clinical investigation, 128(2), 734-745.

Jung, M., Sommer, I., Schachner, M., \& Nave, K. A. (1996). Monoclonal antibody O10 defines a conformationally sensitive cell-surface epitope of proteolipid protein (PLP): evidence that PLP misfolding underlies dysmyelination in mutant mice. J Neurosci, 16(24), 7920-7929.

Kassmann, C. M., Lappe-Siefke, C., Baes, M., Brügger, B., Mildner, A., Werner, H. B., et al. (2007). Axonal loss and neuroinflammation caused by peroxisome-deficient oligodendrocytes. Nature genetics, 39(8), 969-976.

Klugmann, M., Schwab, M. H., Pühlhofer, A., Schneider, A., Zimmermann, F., Griffiths, I. R., et al. (1997). Assembly of CNS myelin in the absence of proteolipid protein. Neuron, 18(1), 5970 . 


\section{Project II}

Kusch, K., Uecker, M., Liepold, T., Mobius, W., Hoffmann, C., Neumann, H., et al. (2017). Partial Immunoblotting of 2D-Gels: A Novel Method to Identify Post-Translationally Modified Proteins Exemplified for the Myelin Acetylome. Proteomes, 5(1).

Lappe-Siefke, C., Goebbels, S., Gravel, M., Nicksch, E., Lee, J., Braun, P. E., et al. (2003). Disruption of Cnp1 uncouples oligodendroglial functions in axonal support and myelination. Nature genetics, 33(3), 366-374.

Lee, Y., Morrison, B. M., Li, Y., Lengacher, S., Farah, M. H., Hoffman, P. N., et al. (2012). Oligodendroglia metabolically support axons and contribute to neurodegeneration. Nature, 487(7408), 443-448.

Lüders, K. A., Nessler, S., Kusch, K., Patzig, J., Jung, R. B., Möbius, W., et al. (2019). Maintenance of high proteolipid protein level in adult central nervous system myelin is required to preserve the integrity of myelin and axons. Glia, 67(4), 634-649.

Lüders, K. A., Patzig, J., Simons, M., Nave, K. A., \& Werner, H. B. (2017). Genetic dissection of oligodendroglial and neuronal Plp1 function in a novel mouse model of spastic paraplegia type 2. Glia, 65(11), 1762-1776.

McKenzie, I. A., Ohayon, D., Li, H., De Faria, J. P., Emery, B., Tohyama, K., et al. (2014). Motor skill learning requires active central myelination. Science, 346(6207), 318-322.

Milner, R. J., Lai, C., Nave, K.-A., Lenoir, D., Ogata, J., \& Sutcliffe, J. G. (1985). Nucleotide sequences of two mRNAs for rat brain myelin proteolipid protein. Cell, 42(3), 931-939.

Möbius, W., Nave, K.-A., \& Werner, H. B. (2016). Electron microscopy of myelin: structure preservation by high-pressure freezing. Brain research, 1641, 92-100.

Moore, S., Meschkat, M., Ruhwedel, T., Tzvetanova, I. D., Trevisiol, A., Battefeld, A., et al. (2019). A role of oligodendrocytes in information processing independent of conduction velocity. bioRxiv, 736975 .

Mukherjee, C., Kling, T., Russo, B., Miebach, K., Kess, E., Schifferer, M., et al. (2020). Oligodendrocytes Provide Antioxidant Defense Function for Neurons by Secreting Ferritin Heavy Chain. Cell Metabolism.

Nave, K.-A. (2010). Myelination and support of axonal integrity by glia. Nature, 468(7321), 244252.

Nave, K.-A., \& Boespflug-Tanguy, O. (1996). X-linked developmental defects of myelination: from mouse mutants to human genetic diseases. The Neuroscientist, 2(1), 33-43.

Nave, K.-A., \& Ehrenreich, H. (2014). Myelination and oligodendrocyte functions in psychiatric diseases. JAMA psychiatry, 71(5), 582-584. 
Nave, K.-A., Lai, C., Bloom, F. E., \& Milner, R. J. (1987). Splice site selection in the proteolipid protein (PLP) gene transcript and primary structure of the DM-20 protein of central nervous system myelin. Proceedings of the National Academy of Sciences, 84(16), 5665-5669.

Nave, K.-A., \& Werner, H. B. (2014). Myelination of the nervous system: mechanisms and functions. Annual review of cell and developmental biology, 30, 503-533.

Nawaz, S., Schweitzer, J., Jahn, O., \& Werner, H. B. (2013). Molecular evolution of myelin basic protein, an abundant structural myelin component. Glia, 61(8), 1364-1377.

Netrakanti, P. R., Cooper, B. H., Dere, E., Poggi, G., Winkler, D., Brose, N., et al. (2015). Fast cerebellar reflex circuitry requires synaptic vesicle priming by munc13-3. The Cerebellum, 14(3), 264-283.

Northoff, G., Kötter, R., Baumgart, F., Danos, P., Boeker, H., Kaulisch, T., et al. (2004). Orbitofrontal cortical dysfunction in akinetic catatonia: a functional magnetic resonance imaging study during negative emotional stimulation. Schizophrenia bulletin, 30(2), 405-427.

O'Connor, A. M., Burton, T. J., Leamey, C. A., \& Sawatari, A. (2014). The use of the puzzle box as a means of assessing the efficacy of environmental enrichment. JoVE (Journal of Visualized Experiments)(94), e52225.

Patzig, J., Kusch, K., Fledrich, R., Eichel, M. A., Lüders, K. A., Möbius, W., et al. (2016). Proteolipid protein modulates preservation of peripheral axons and premature death when myelin protein zero is lacking. Glia, 64(1), 155-174.

Pease-Raissi, S. E., \& Chan, J. R. (2018). Micro (glial)-managing executive function: white matter inflammation drives catatonia. The Journal of clinical investigation, 128(2), 564-566.

Peters, A., \& Kemper, T. (2012). A review of the structural alterations in the cerebral hemispheres of the aging rhesus monkey. Neurobiology of aging, 33(10), 2357-2372.

Peters, A., \& Sethares, C. (2002). The effects of age on the cells in layer 1 of primate cerebral cortex. Cerebral Cortex, 12(1), 27-36.

Petit, B., Giraudet, F., Béchon, C., Bardin, L., Avan, P., Boespflug-Tanguy, O., et al. (2014). Mice with a deletion of the major central myelin protein exhibit hypersensitivity to noxious thermal stimuli: involvement of central sensitization. Neurobiology of disease, 65, 55-68.

Poggi, G., Boretius, S., Möbius, W., Moschny, N., Baudewig, J., Ruhwedel, T., et al. (2016). Cortical network dysfunction caused by a subtle defect of myelination. Glia, 64(11), 2025-2040. Rosenbluth, J., Nave, K. A., Mierzwa, A., \& Schiff, R. (2006). Subtle myelin defects in PLP-null mice. Glia, 54(3), 172-182. 


\section{Project II}

Saab, A. S., Tzvetavona, I. D., Trevisiol, A., Baltan, S., Dibaj, P., Kusch, K., et al. (2016). Oligodendroglial NMDA receptors regulate glucose import and axonal energy metabolism. Neuron, 91(1), 119-132.

Saugier-Veber, P., Munnich, A., Bonneau, D., Rozet, J.-M., Le Merrer, M., Gil, R., et al. (1994). $\mathrm{X}$-linked spastic paraplegia and Pelizaeus-Merzbacher disease are allelic disorders at the proteolipid protein locus. Nature genetics, 6(3), 257.

Schardt, A., Brinkmann, B. G., Mitkovski, M., Sereda, M. W., Werner, H. B., \& Nave, K.-A. (2009). The SNARE protein SNAP-29 interacts with the GTPase Rab3A: implications for membrane trafficking in myelinating glia. Journal of Neuroscience Research, 87, 3465-3479.

Snaidero, N., Velte, C., Myllykoski, M., Raasakka, A., Ignatev, A., Werner, H. B., et al. (2017). Antagonistic functions of MBP and CNP establish cytosolic channels in CNS myelin. Cell reports, 18(2), 314-323.

Stassart, R. M., Möbius, W., Nave, K.-A., \& Edgar, J. M. (2018). The axon-myelin unit in development and degenerative disease. Frontiers in neuroscience, 12, 467.

Steyer, A. M., Ruhwedel, T., Nardis, C., Werner, H. B., Nave, K.-A., \& Möbius, W. (2020). Pathology of myelinated axons in the PLP-deficient mouse model of spastic paraplegia type 2 revealed by volume imaging using focused ion beam-scanning electron microscopy. Journal of structural biology, 210(2), 107492.

Trapp, B. D., Nishiyama, A., Cheng, D., \& Macklin, W. (1997). Differentiation and death of premyelinating oligodendrocytes in developing rodent brain. The Journal of cell biology, 137(2), 459-468.

Trevisiol, A., Kusch, K., Steyer, A. M., Gregor, I., Nardis, C., Winkler, U., et al. (2020). Structural myelin defects are associated with low axonal ATP levels but rapid recovery from energy deprivation in a mouse model of spastic paraplegia. PLoS biology, 18(11), e3000943.

Trevisiol, A., Saab, A. S., Winkler, U., Marx, G., Imamura, H., Möbius, W., et al. (2017). Monitoring ATP dynamics in electrically active white matter tracts. Elife, 6, e24241.

Wang, L., Winnewisser, J., Federle, C., Jessberger, G., Nave, K.-A., Werner, H. B., et al. (2017). Epitope-specific tolerance modes differentially specify susceptibility to proteolipid proteininduced experimental autoimmune encephalomyelitis. Frontiers in immunology, 8, 1511.

Zhou, B., Zhu, Z., Ransom, B.R., and Tong, X. (2021). Oligodendrocyte lineage cells and depression. Mol. Psych. 26, 103-117. 


\section{Acknowledgments}

We thank A. Sasmita, A. Fahrenholz and R. Jung for technical support and E.-M. KrämerAlbers for antibodies.

\section{Funding}

Our work is supported by the Deutsche Forschungsgemeinschaft (DFG) (WE 2720/2-2, WE 2720/4-1 and WE 2720/5-1 to H.B.W.) and the European Research Council (ERC) (advanced grant 'MyeliNano' to K.-A.N.).

\section{Conflict of interest statement}

The authors declare no conflict of interest 


\section{Project II}

\section{FIGURE LEGENDS}

\section{Figure 1. Targeting PLP expression in the forebrain of mice}

(A,B) Light-sheet microscopy to detect proteolipid protein (PLP) in the brains of Plp $p^{f a x} / Y$ (Ctrl; A) and $E m x^{C r}:: P / p^{f b x} / Y(c K O ; B)$ mice. Whole mount brain sections $(1 \mathrm{~mm})$ were subjected to staining and clearing. Boxed areas in panels $\mathbf{A}$ and $\mathbf{B}$ are enlarged in $\mathbf{A}^{\mathbf{s}}, \mathbf{A}^{\boldsymbol{⿰}}$ and $\mathbf{B}^{\mathbf{s}}, \mathbf{B}^{\star}$, respectively. White arrows indicate the sparse labeling of PLP in forebrain cortical structures (B') and the hippocampus (B') of cKO in comparison to controls (A', A'). PLP, proteolipid protein; CNP, 2'-3'-cyclic nucleotide 3'-phosphodiesterase; Autofl, autofluorescence.

(C) Immunoblot detection of PLP and its smaller isoform DM20 in lysates of the prefrontal

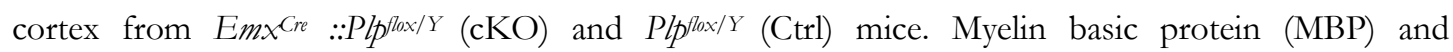
actin were used as loading controls ( $\mathrm{n}=2$ biological replicates). (D) Immunoblots of the cerebellum from the same mice.

Figure 2. Neurodegenerative changes in the subcortical white matter of the forebrain (A,C,E,G) Representative light microscopic images of cross-sectioned hippocampal fimbriae, immunolabeled for (A) APP, (C) MAC3, (E) IBA1 and (G) GFAP in Em $x^{C r e:: P / p^{f o x} / Y}(\mathrm{cKO})$ and $P / p^{f o x} / Y$ (Ctrl) mice at age 22 months. The frequency of axonal spheroids and the area of immunopositivity for MAC3, IBA1 and GFAP are increased in the fimbria of cKO compared to Ctrl mice as quantified in $\mathbf{B}, \mathbf{D}, \mathbf{F}$ and $\mathbf{H}$. Scale bars $50 \mu \mathrm{m}$. (I,J) Representative light microscopic images of cross-sectioned $\mathrm{Ctrl}$ (I) and cKO (J) mouse brains. Boxed areas in panels $\mathbf{I}$ and $\mathbf{J}$ are enlarged in I', I' and $\mathbf{J}$, J', respectively. Black arrows indicate axonal spheroids in the fimbria (J') and internal capsule (J') of cKO mice, which are essentially absent in Ctrl mice (I', I'). Mean +/ SEM; two-tailed unpaired t-test; ${ }^{* *}, \mathrm{p}<0.01 ; * * *, p<0.001$. APP, amyloid precursor protein; CC, corpus callosum; Ctx, cortex; Fim, fimbria; GFAP, glial fibrillary acidic protein; IBA1, ionized calcium-binding adaptor molecule 1 MAC3, lysosomalassociated membrane protein 2 (LAMP2).

Figure 2-supplement 1. Neuropathology in the forebrain of cKO mice in the corpus callosum but not the prefrontal cortex

(A,C,E,G) Representative light microscopic images of cross-sectioned corpus callosum immunolabeled for APP (A), MAC3 (C), IBA1 (E) and GFAP (G) in Plp $p^{f l a x / Y *} E m x^{C r e}(\mathrm{cKO})$ and $P \not p^{f l o x / Y}$ (Ctrl) mice at age 22 months. Scale bars $50 \mu \mathrm{m}$.

$\mathbf{( B , D , F , H ) ~ G e n o t y p e - d e p e n d e n t ~ q u a n t i f i c a t i o n ~ o f ~ t h e ~ f r e q u e n c y ~ o f ~ A P P - i m m u n o p o s i t i v e ~}$ axonal spheroids in the corpus callosum (B) and the relative area of the fimbria immunopositive for the microglial markers MAC3 (D) or IBA1 (F) or the astroglial marker GFAP (H). Note 
that the frequency of axonal spheroids and the area of immunopositivity for MAC3, IBA1 and GFAP are increased in the corpus callosum of cKO compared to Ctrl mice.

(I,K,M,O) Representative light microscopic images of cross-sectioned prefrontal cortex immunolabeled for APP (I), MAC3 (K), IBA1 (M) and GFAP (O) in Plp flax/Y*Emx $x^{\text {Cre }}(\mathrm{cKO})$ and $P l p^{f t a x / Y}$ (Ctrl) mice at age 22 months. Scale bars $50 \mu \mathrm{m}$.

$(\mathbf{J}, \mathbf{L}, \mathbf{N}, \mathbf{P})$ Genotype-dependent quantification of the frequency of APP-immunopositive axonal spheroids in the prefrontal cortex $(\mathbf{J})$ and the relative area of the fimbria immunopositive for the microglial markers MAC3 (L) or IBA1 (N) or the astroglial marker GFAP (P). Note that the frequency of axonal spheroids and the area of immunopositivity for MAC3, IBA1 and GFAP are similar in the prefrontal cortex of cKO compared to Ctrl mice. Mean + / SEM; twotailed unpaired t-test; not significant, n.s.; ${ }^{*}, \mathrm{p}<0.05 ;{ }^{* *}, \mathrm{p}<0.01$.

Figure 3. cKO mice display a catatonia-like sign along with impaired executive function $(\mathbf{A}, \mathbf{B})$ Bar test assessing the time that mice remain immobile upon placement of their forelimbs on a horizontal bar. Female (A) and male (B) groups of cKO and Ctrl mice were tested the indicated ages. Note that $\mathrm{cKO}$ mice upon ageing spend extended time immobile on the bar. Mean +/ SEM; Mann-Whitney- $U$-test. (C,D) Puzzle box assessing the time to escape from a well-lit open space into a dark shelter with increasing difficulty (open entry, addition of a gateway, gateway filled with bedding, entry filled with paper tissue, entry filled with nesting material; see illustrating images in Figure 3-supplement 1). Female (C) and male (D) groups of cKO and Ctrl mice were assessed at age 20 or 18 months, respectively. Mean +/- SEM; repeated measures 2-way ANOVA; females significant ***, $\mathrm{p}<0.001$; a trend to increased latency for males did not reach significance, n.s., $\mathrm{p}=0.0518$. (E,F) Modified Hurdle test assessing the escape latency of mice by crossing $5 \mathrm{~cm}$ hurdles upon placement into the center of a circular open field arena. Female (E) and male (F) mice were assessed at the age of 6 and 20 months. Mean + / SEM; Mann-Whitney-U-test; Trial 1 or 2, T1 or T2. All data in this figure are presented as mean +/- SEM; $\mathrm{p}<0.05$ considered significant. The precise number of mice tested per experiment, mean, SEM and p-values are specified in supplemental Table 1. 


\section{Project II}

Figure 3-supplement 1. Puzzle box

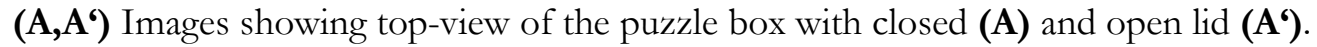

(B-F) Front-view of the shelter with open entry (B), entry with gateway (C), gateway filled with bedding (D), entry filled with paper tissue (E) and entry filled with nesting material (F).

Figure 3-supplement 2. cKO mice display normal latency to fall on the Rotarod

(A-D) Rotarod test assessing motor performance of cKO and Ctrl mice at ages 6 months $(\mathbf{A}, \mathbf{B})$ and 17 months (C,D) on two consecutive days (day 1 and day 2). Female (A,C) and male (B,D) groups were assessed. Note that cKO mice performed normally on the Rotarod. Mean +/SEM; not significant (n.s.) by repeated measures 2-way ANOVA.

Figure 4. cKO mice display reduced running distance but normal velocity

(A-F) Complex running wheel (CRW) assessing voluntary running behavior. Female (A,C,E) and male (B,D,F) groups of cKO and Ctrl mice were assessed at age 23 months. Both female and male cKO mice displayed reduced running distance $(\mathbf{A}, \mathbf{B})$ but normal average running velocity $(\mathbf{E}, \mathbf{F})$. A trend toward reduced running time reached significance in female but not in male cKO mice (C,D). Note that the result is interpreted as reflecting that $\mathrm{cKO}$ mice show normal motor capability but impaired executive function. Superimposed plot with connecting lines; repeated measures 2-way ANOVA; not significant, n.s.; * $\mathrm{p}<0.05$. 


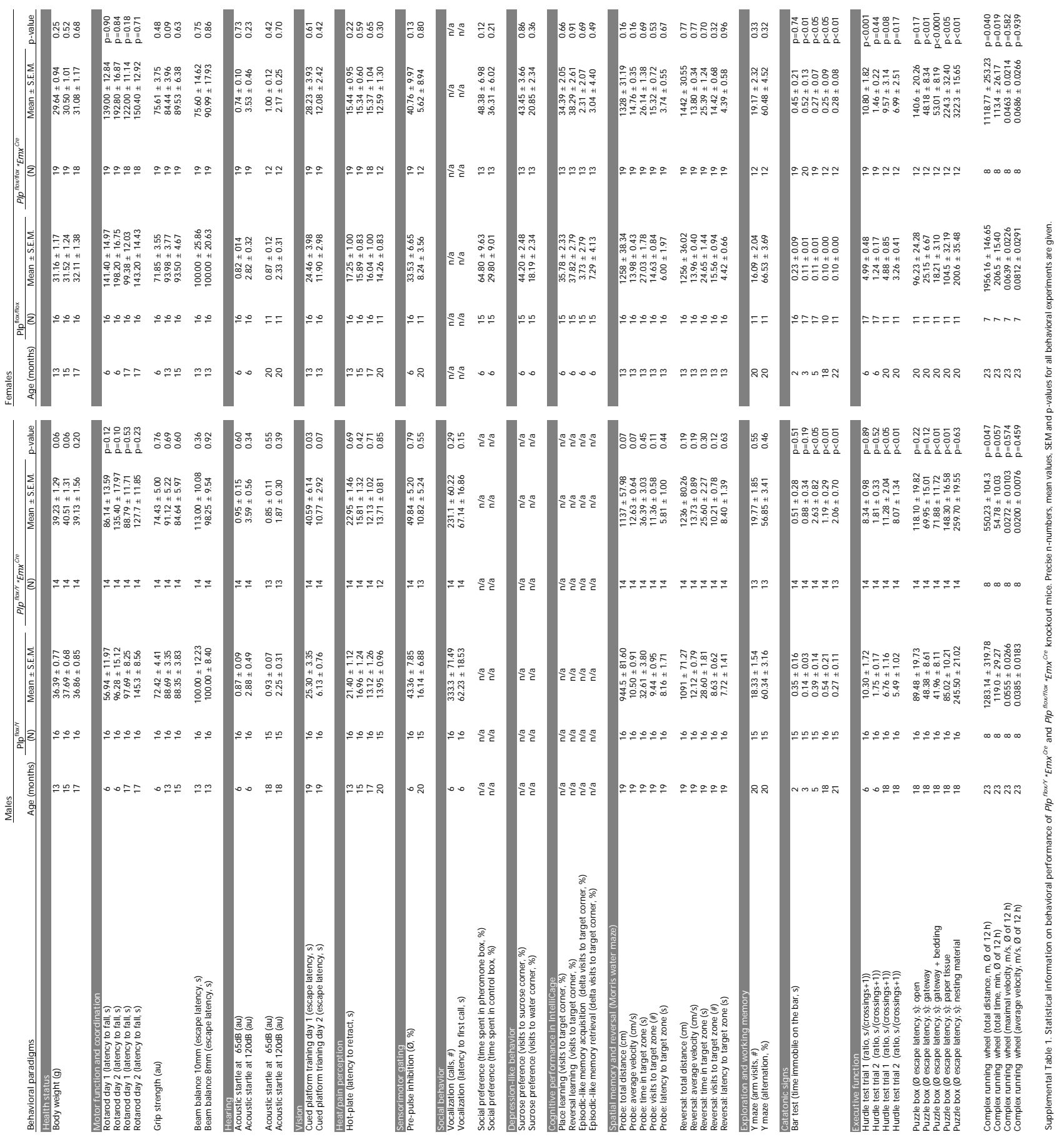


Arinrad et al., Figure 1
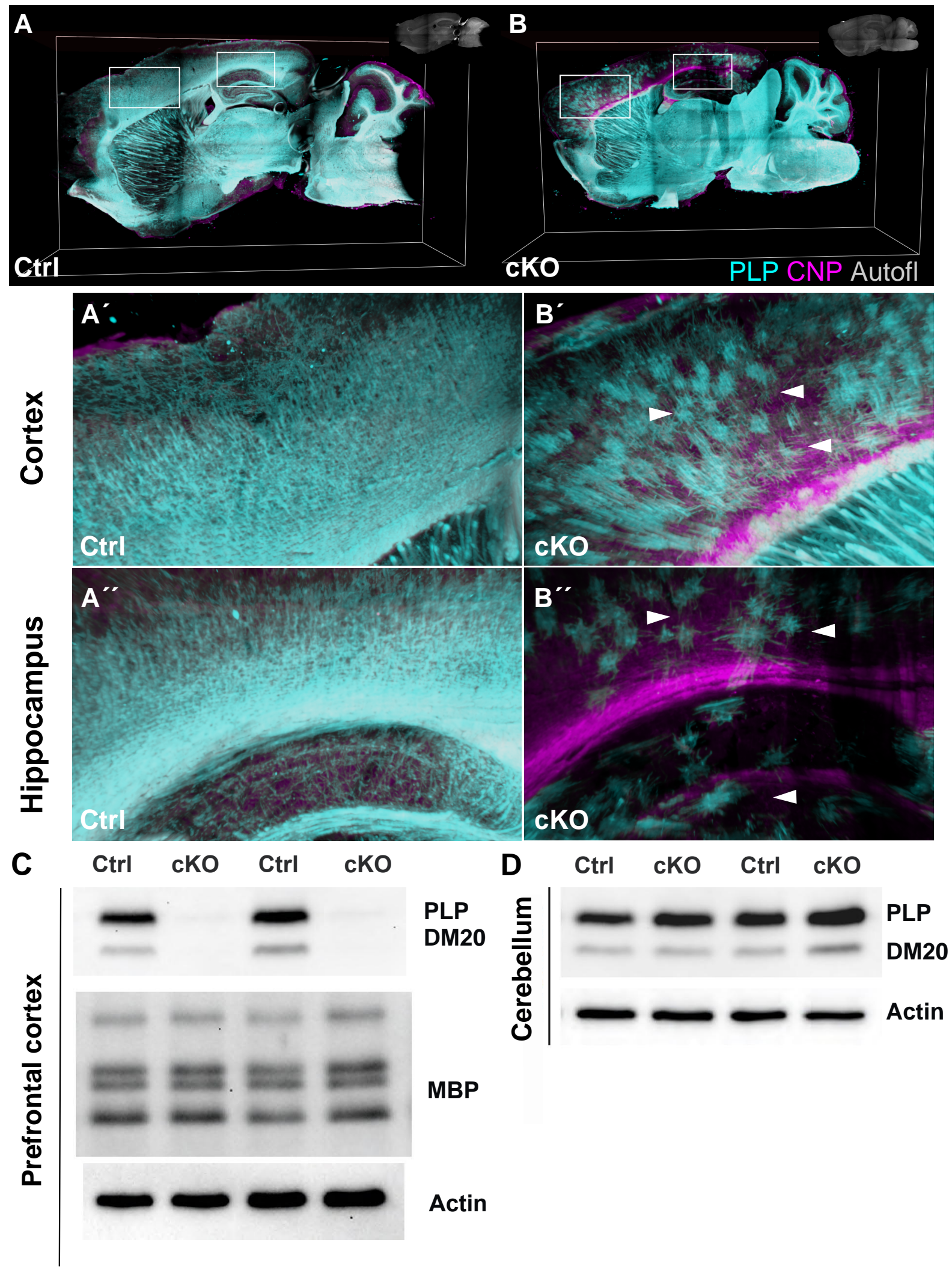
Project II

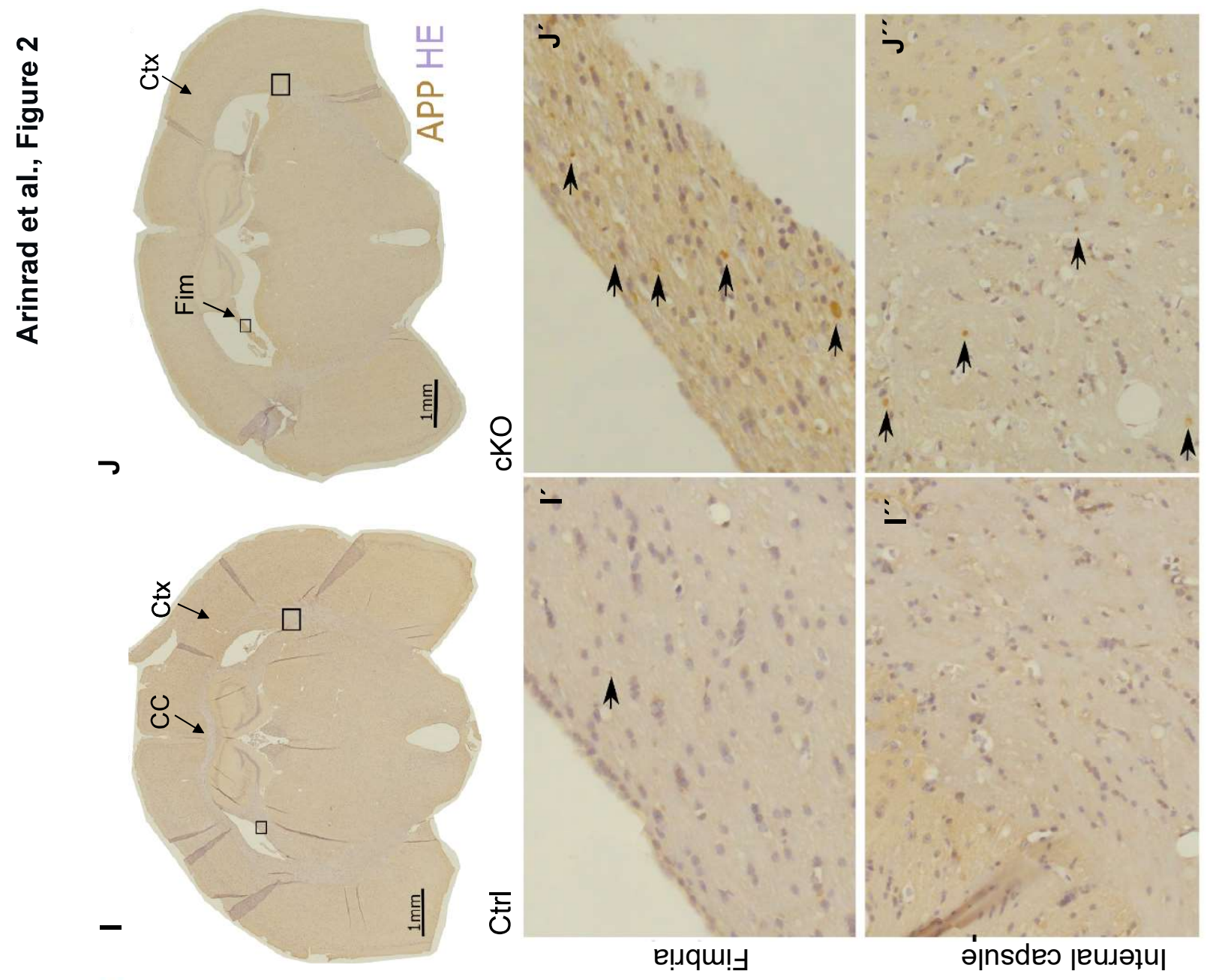

통 움

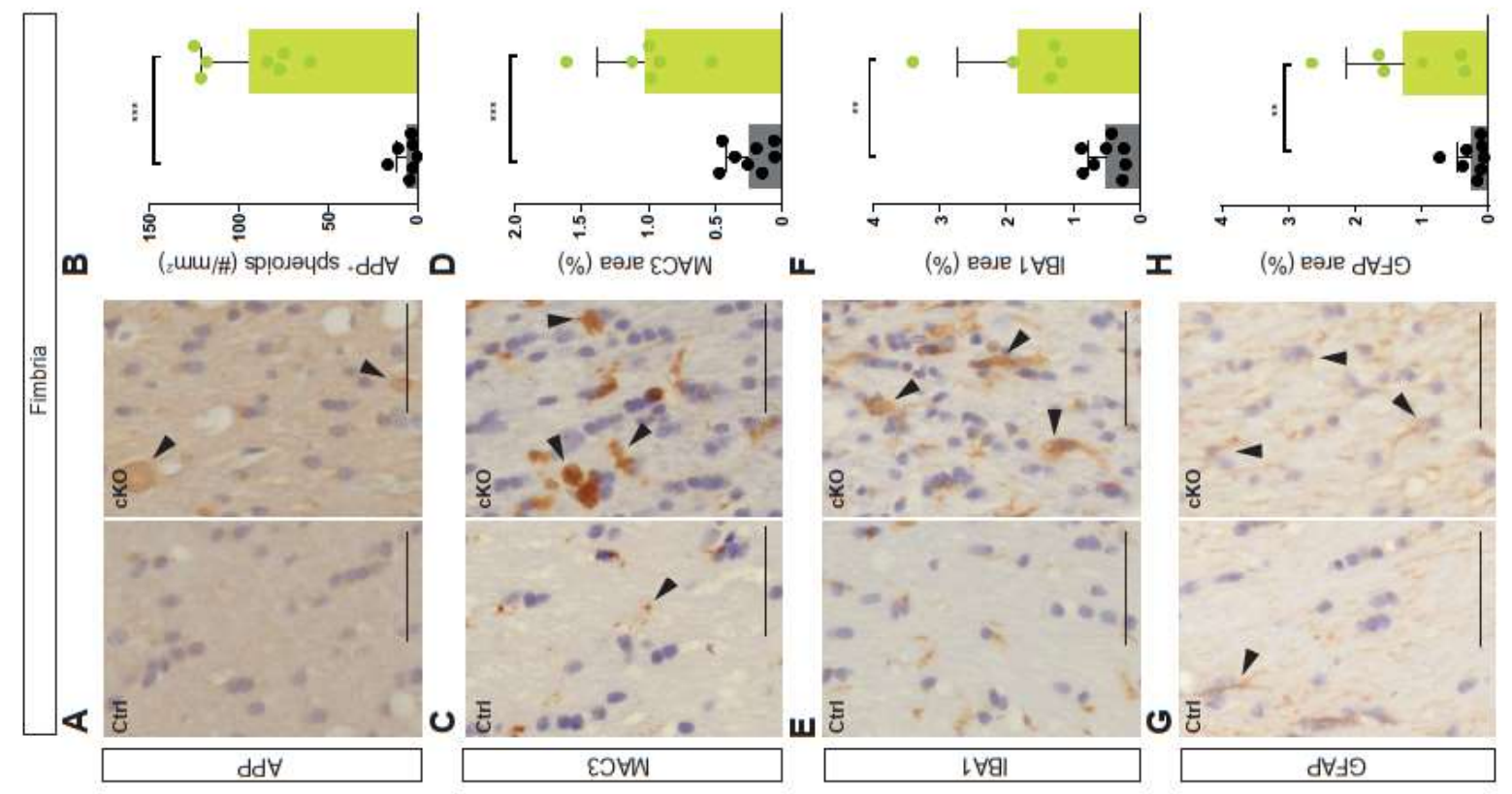




\section{Project II}

Arinrad et al., Figure 3
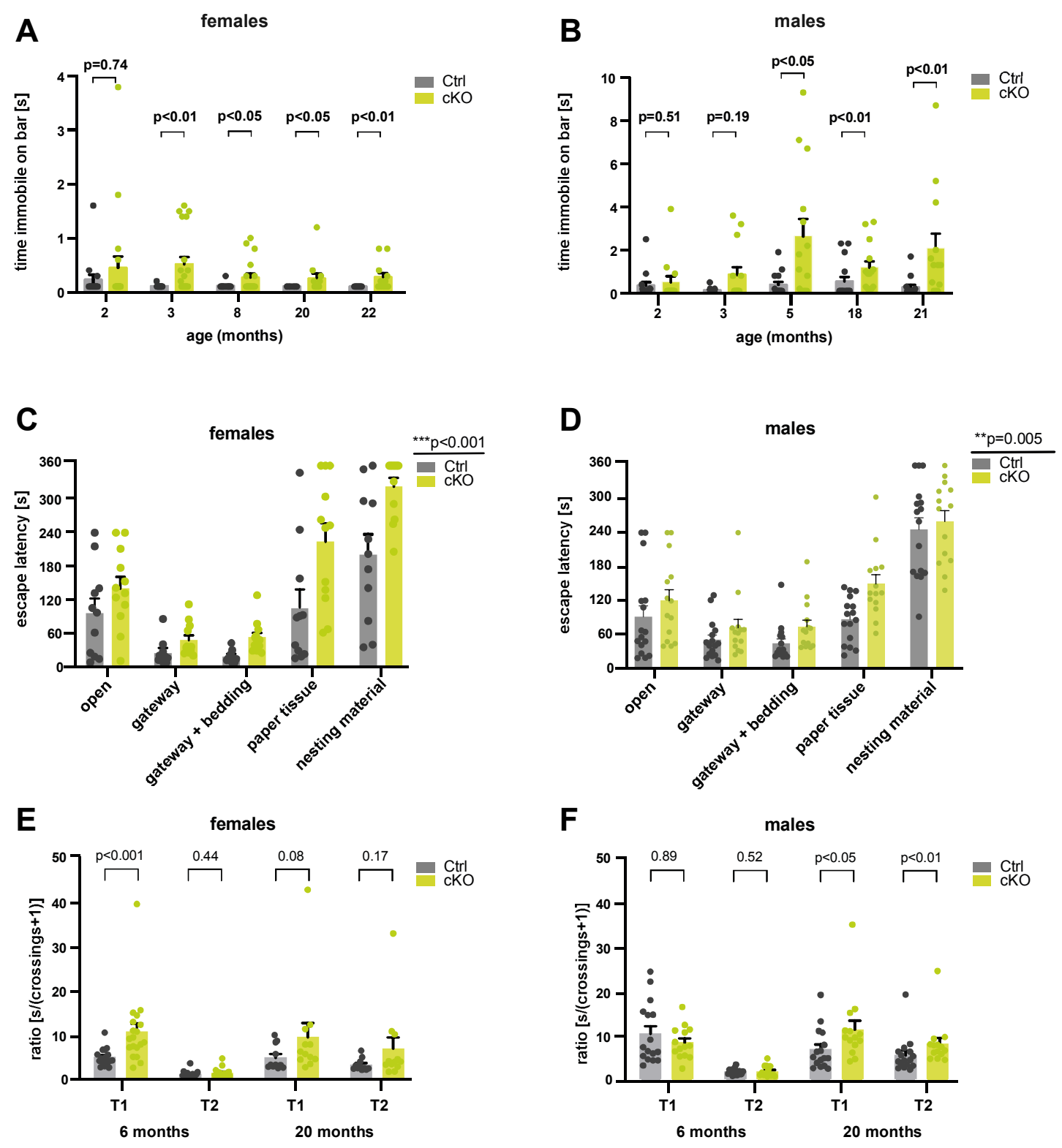


\section{Arinrad et al., Figure 4}

A
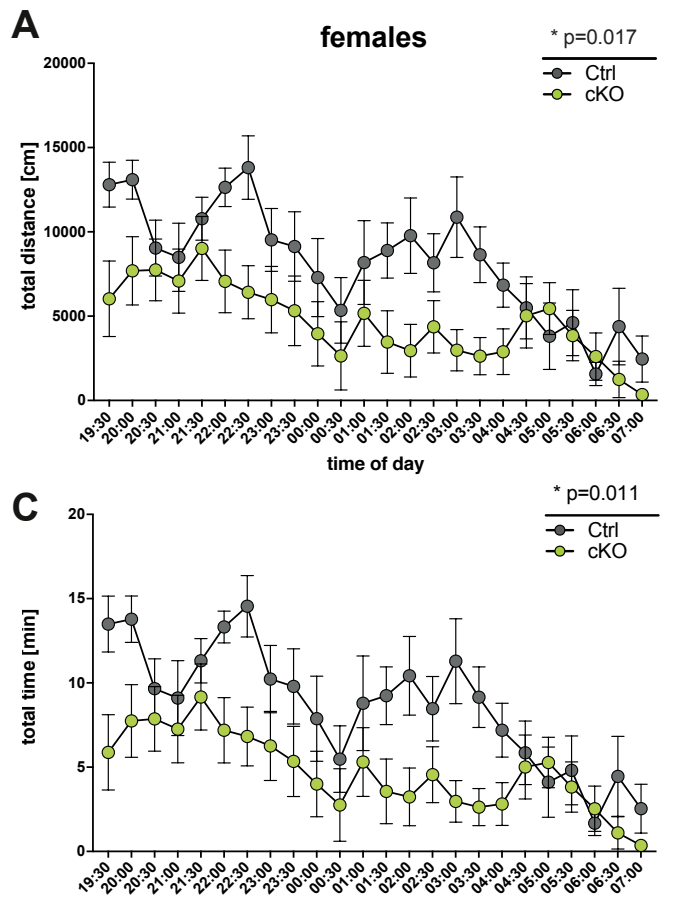

time of day
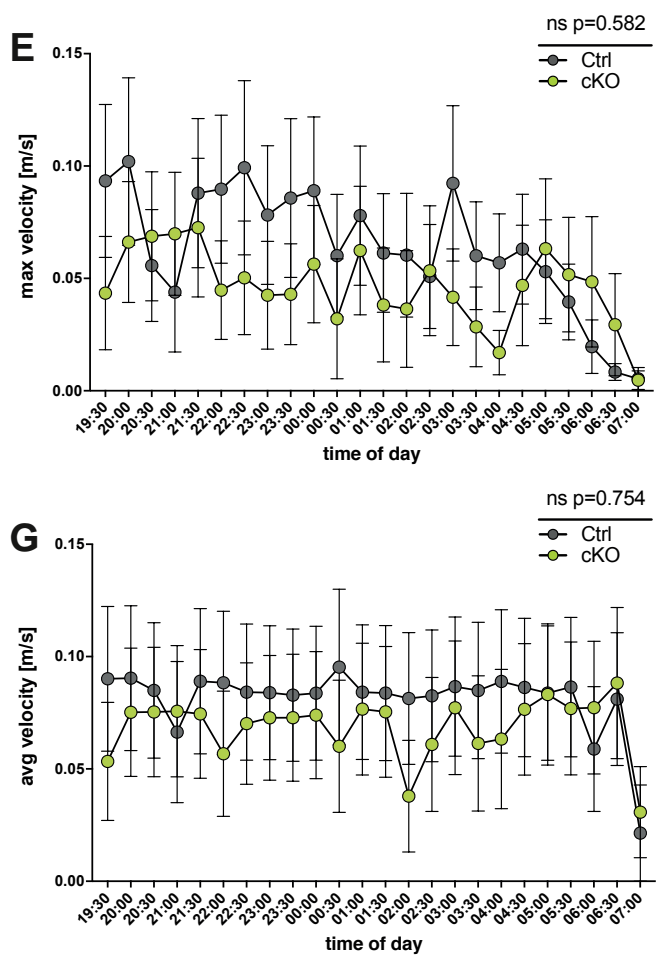

B

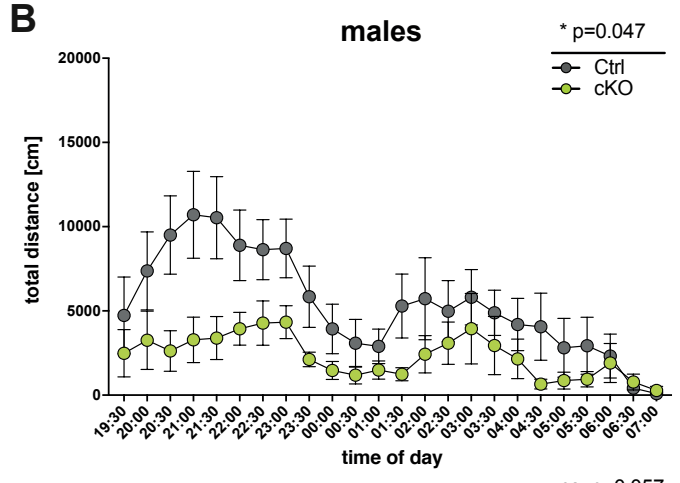

D
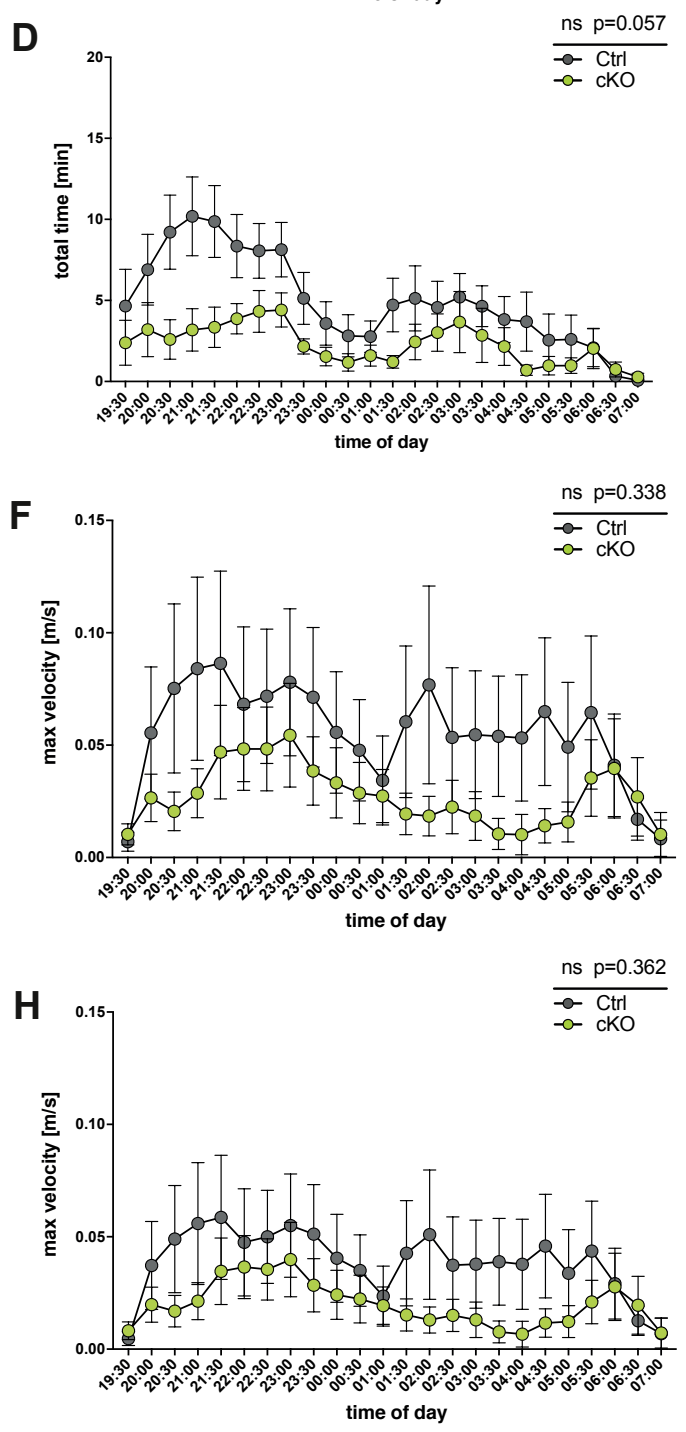


\section{Project II}
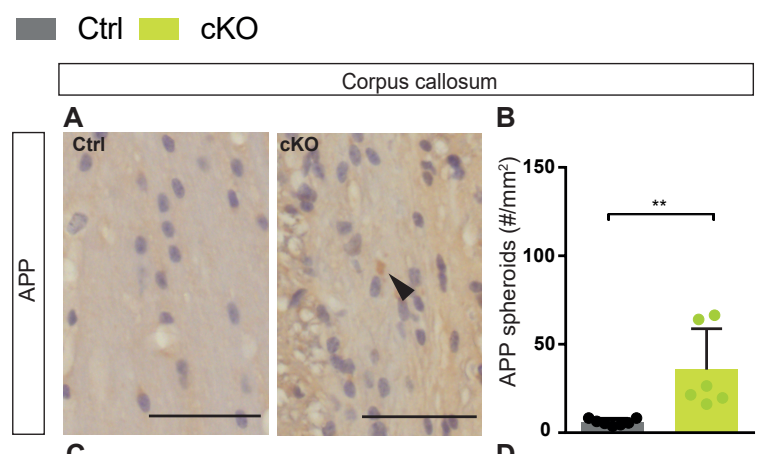

c

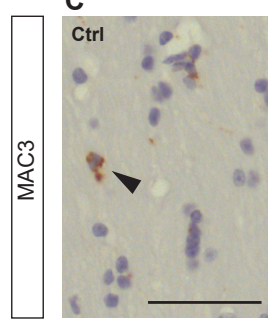

E

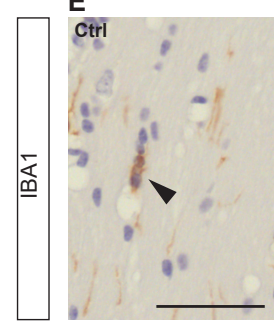

G

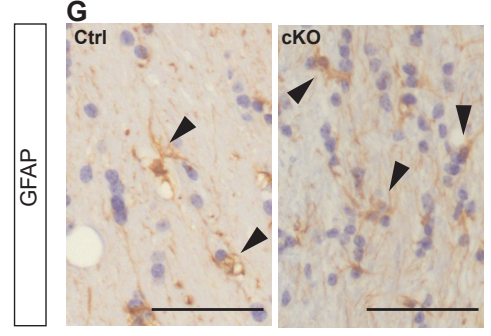

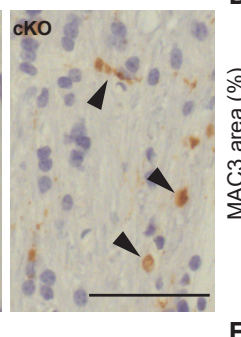

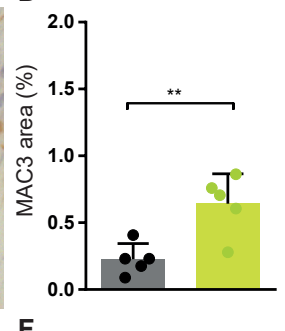

$\mathrm{F}$

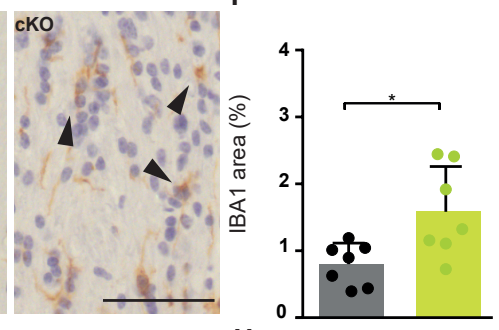

$\mathrm{H}$

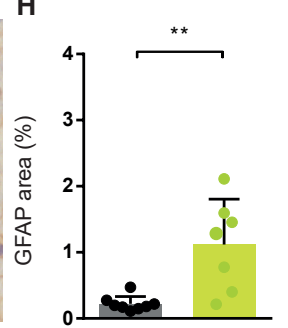

Arinrad et al., Figure 2 - supplement 1
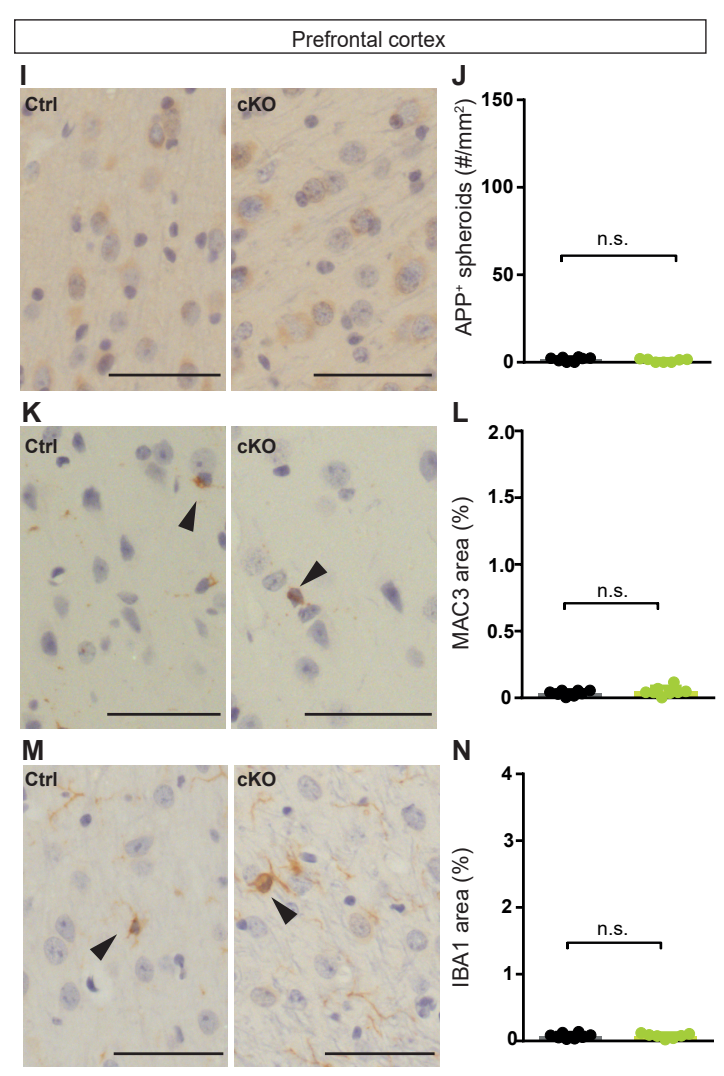

O
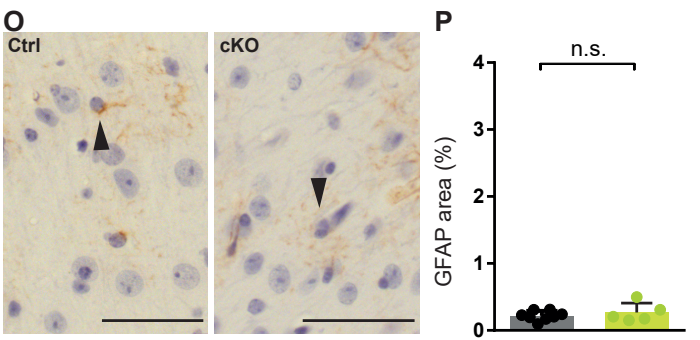

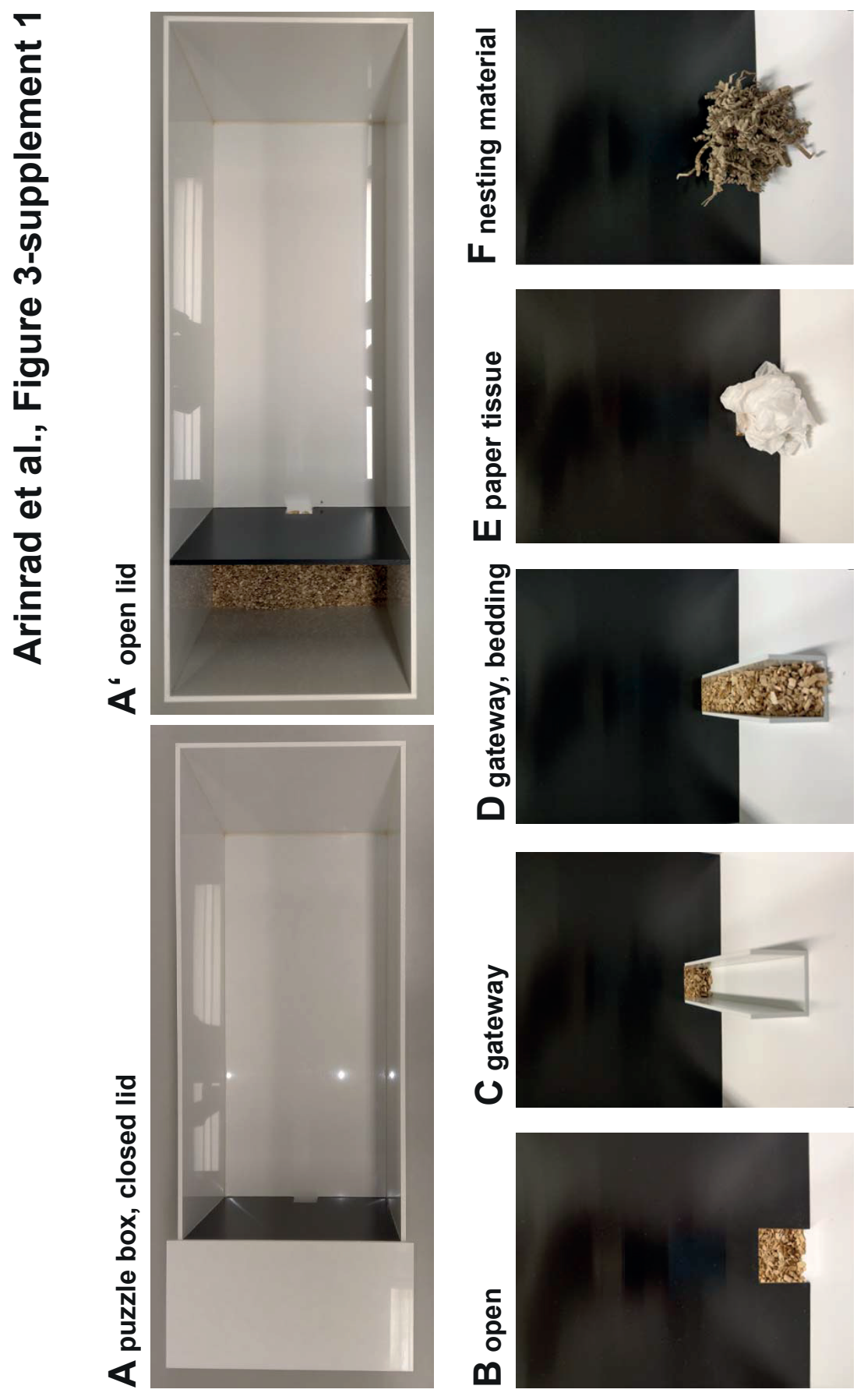


\section{Project II}

Arinrad et al., Figure 3 - supplement 2
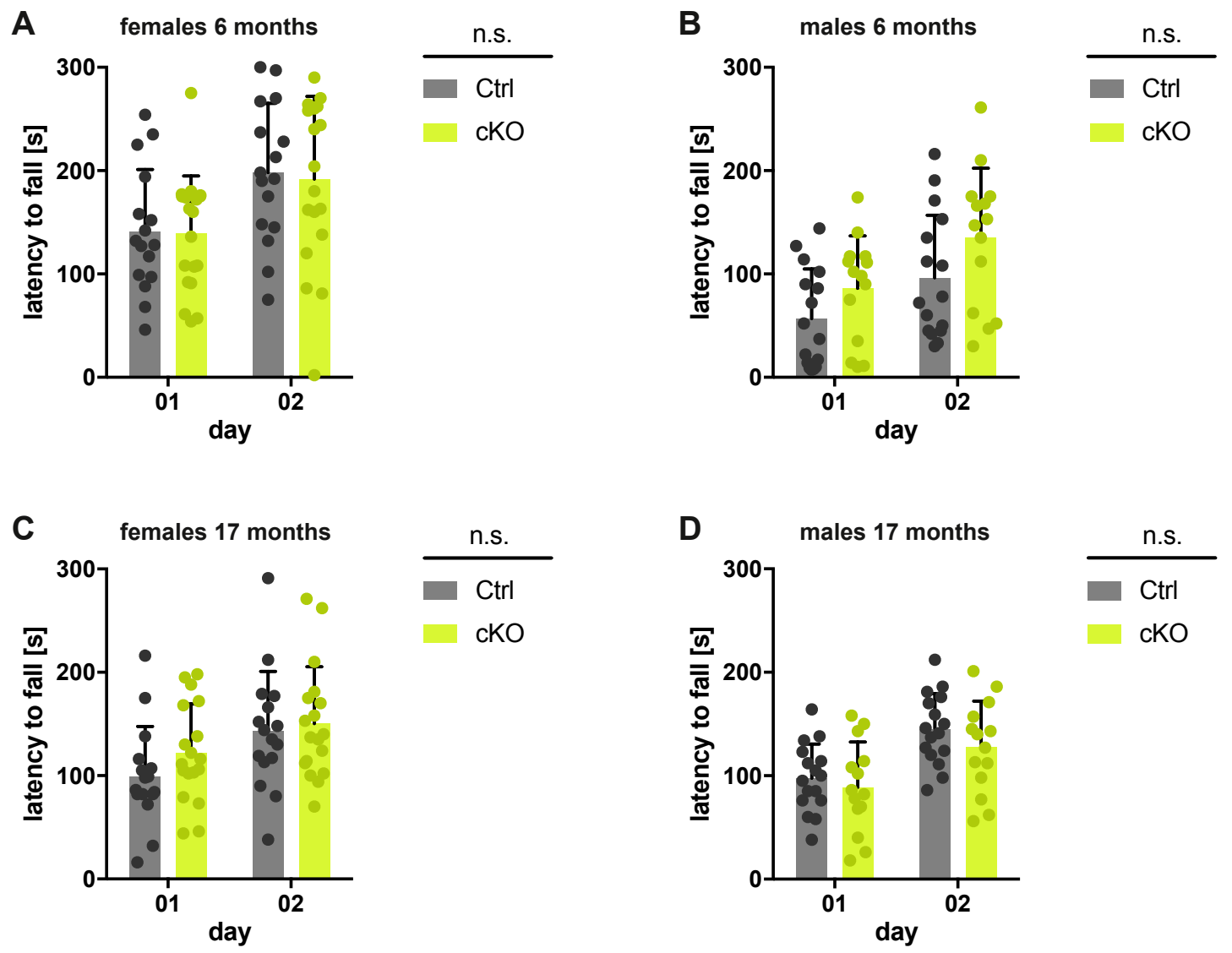


\section{Chapter 4}

\section{Conclusions \& Future Perspectives}


Conclusions \& Future Perspectives 


\section{Conclusions \& Future Perspectives}

\section{Conclusions and Future Perspectives}

Despite its first description by Kahlbaum (1874) as a novel psychomotor syndrome, the clinical diagnosis of catatonia and its respective treatment have been hampered for more than a century. The inclusion of catatonia as a subtype of dementia praecox (Kraepelin, 1899), and later on schizophrenia (Bleuler, 1911), have largely contributed to conceptual limitations and misperceptions of the syndrome. Today, catatonia is considered an independent psychomotor syndrome, presenting with disturbed volition along with aberrant motor and behavioral phenotypes (Hirjak et al., 2020). However, its etiology and underlying pathomechanisms still remain unclear and efficient treatment scarce. Moreover, research on catatonia is confined by divided clinical rating scales (Bräunig et al., 2000; McKenna et al., 1991; Northoff, 2000; Northoff, Koch, et al., 1999) and particularly by the lack of reliable animal models.

Previous studies from our group implied involvement of low-grade neuroinflammation as well as altered white matter integrity in $\mathrm{C} 57 \mathrm{Bl} / 6$ mice deficient of the myelin genes Cnp (Hagemeyer et al., 2012) or Mbp (Poggi et al., 2016) upon aging. A comparable age-dependent catatoniadepression syndrome was further reported in human schizophrenic carriers of a partial loss-offunction SNP in the CNP gene (Hagemeyer et al., 2012). Based on these findings, I aimed to systematically investigate the revelance of white matter integrity and neuroinflammatory processes in the context of the catatonia-like phenotype, with a particular focus on frontal brain structures, known to be essential to healthy executive function and higher-order cognitive abilities (Alvarez \& Emory, 2006; Diamond, 2013; Stuss \& Benson, 1984).

\section{Increased signs of neuroinflammation and catatonic signs in schizophrenic patients carrying the the $C N P$ partial loss-of-function SNP rs2070106-AA}

Upon first indications of neuropathology by DTI in schizophrenic carriers of the CNP SNP rs2070106-AA (Hagemeyer et al., 2012), we conducted a GWAS on the baseline cohort of SHIP-TREND-0 in close collaboration with Prof. Dr. Hans Jörgen Grabe and co-workers. We assessed the presence and extent of WMH in healthy subjects with and without the CNP SNP. An age-dependent increase in WMH was observed, confirming previous reports on the progression of WMH and its impact on white matter integrity even affecting cognitive abilities (Almkvist et al., 1992; Garde et al., 2005; Sullivan et al., 2001). Interestingly, WMH in our healthy subjects were significantly exceeded by carriers of the SNP, particularly affecting frontotemporal brain regions. While these data do not provide eminent proof of increased neuroinflammation 


\section{Conclusions \& Future Perspectives}

in CNP rs2070106-AA carriers, we previously also reported increased signs of degenerative processes in the frontal CC of AA carriers by DTI (Hagemeyer et al., 2012). These findings not only confirm a dominant phenotype in frontal brain stuctures, but further imply an underlying neuropathology.

Moreover, application of another GWAS study using our GRAS database on schizophrenic subjects (Begemann et al., 2010; Ribbe et al., 2010) revealed a significant age-dependent increase in catatonic signs, assessed by the CNI catatonia score (Chen et al., 1995), in $A A$ carriers of the SNP. Noteworthy, this finding was confirmed in an independent sample of schizophrenic subjects in close collaboration with Prof. Dr. Andreas Reif and co-workers. Collectively, these data are not only in accordance with our previous reports on the impact of the $A A$ genotype on catatonic signs in schizophrenic subjects (Hagemeyer et al., 2012), but further suggest underlying neuroinflammatory processes and altered white matter integrity to be causative to the observed phenotype. Systematic analysis of postmortem tissue of schizophrenic subjects comparing $A A$ carriers with $G G$ and $A G$ carriers - would be helpful to further evaluate the extent and distribution of the underyling neuropathology. Additionally, state of the art neuroimaging techniques provide an essential tool aiming to uncover the underlying neuroanatomical and biochemical causes of catatonia (Hirjak et al., 2020).

\section{Neuroinflammation of subcortical white matter is causative to a catatonia- like phenotype in $C n p^{-/-}$mice}

Based on previous observations of an age-dependent catatonia-like phenotype in C57Bl/ 6 mice with a heterozygous deficiency of the myelin genes Cnp (Hagemeyer et al., 2012) or Mbp (Poggi et al., 2016), I aimed to delineate the relevance of white matter integrity per se in the context of catatonia. To this end, we observed a striking facilitation of the onset of catatonic signs, assessed by a modified version of the well-established Bar test (Hagemeyer et al., 2012; Kuschiski et al., 1972; Poggi et al., 2016), in $C n p^{--}$mice. In contrast to $C n p^{+/-}$mice, $C n p^{-/-}$exhibit a significantly prolonged time standing immobile on a horizontal rod as early as 8 weeks of age. Importantly, this phenotype occurs in the absence of any motor dysfunctions, known to develop at progressed age upon CNP deficiency, including ataxia, hindlimb impairments and even premature death (Lappe-Siefke et al., 2003). Thus, we suggest the inability of $C n p^{-1-}$ mice to release themselves from an imposed unnatural posture to likely reflect a transient executive dysfuntion but no underlying motor impairments or forelimb paralysis. 


\section{Conclusions \& Future Perspectives}

To assess the distinct relevance of myelin-related alterations regarding the observed phenotype, I conducted the Bar test on $M b p^{+/-}$mice, the parental strain of shiverers (Chernoff, 1981; Readhead et al., 1987), as well as $P / p^{-1 y}$ mice, a well-characterized mouse model of SPG2 (Griffiths et al., 1998; Lüders et al., 2017; Saugier-Veber et al., 1994), both of which indeed exhibited catatonic signs at the ages of 60-72 or 25 weeks, respectively. Importantly, analysis of various other mouse models of CNS disease, including $\mathrm{Ambra1}^{+/-}$(Dere et al., 2014), Nlgn4\% (Jamain et al., 2008), Caps1\% (Jockusch et al., 2007), PSD93\% and PSD95\% (Winkler et al., 2018) and $A p o E^{-/-}$(Hammer et al., 2014) mice did not result in any of these lines exhibiting catatonic signs in the Bar test. While I cannot exclude the presence of a similar phenotype in other mouse models of CNS diseases, our data reliably suggest a key involvement of myelin-related genes and (subtle) alterations of such to be causative to catatonia.

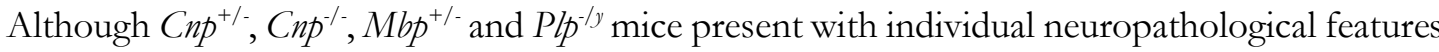
(Griffiths et al., 1998; Hagemeyer et al., 2012; Lappe-Siefke et al., 2003; Poggi et al., 2016), they all share the development of progressive neuroinflammation, however with onset of such at distinct ages. The latter is predominantly caused by abberant microglial phenotypes, shifting into detrimental and neurotoxic activities (Graeber et al., 2011; Streit et al., 2004). Microglial contributions to neurological and neuropsychiatric disorders are of increasing interest in recent years (Ransohoff \& El Khoury, 2016; Salter \& Stevens, 2017), ultimately aiming to address the question whether microglial dysfunctions are the cause or the consequence of neuropathology. Noteworhty, neuroinflammatory processes in the catatonia-like phenotype of $\mathrm{Cnp}^{+/-}$and $\mathrm{Mbp}^{+/}$ mice have been reported before (Hagemeyer et al., 2012; Poggi et al., 2016). Thus, I aimed to tackle the question of their importance to the development of the phenotype, particularly by application of PLX5622, a CSF1-R inhibitor causing rapid depletion of microglia, while allowing swift repopulation upon cessation of treatment (Dagher et al., 2015).

Indeed, treatment of $\mathrm{Cnp}^{-1}$ mice prior to the onset of the phenotype prevented it from occurring at a young age, i.e. 8 weeks. IHC analysis of the CC revealed a surprisingly sustained reduction of both neuroinflammation $\left(\mathrm{IBA}^{+}\right)$and -degeneration $\left(\mathrm{APP}^{+}\right)$, known features of $\mathrm{Cnp}^{-/}$mice (Lappe-Siefke et al., 2003), even 4 weeks after cessation of treatment. The beneficial impact of microglia-targeted treatment to the phenotype was further confirmed by MRS. We report a reduction fo myoinositol, a marker of glial activation and neuroinflammation, to be reduced in the CC and cortex of PLX5622-treated $C n p^{-1-}$ mice, even 5 weeks after cessation of treatment. The beneficial impact of microglia-targeted treatment, on a phenotype deriving from disrupted white matter integrity, is in accordance with another study, reporting attenuation of neuropathology induced by mutations of another myelin gene, namely PLP1 (Groh et al., 2019). 


\section{Conclusions \& Future Perspectives}

In recent years, white matter integrity and microglial activities, as well as their interactions, have gained an increasing attention in the context of healthy CNS functions (Gomez-Nicola et al., 2015; Nave \& Ehrenreich, 2014; Ransohoff \& El Khoury, 2016; Tay et al., 2018). Moreover, alterations of both appear to play an intertwined role in the development and progression of CNS disorders (Lloyd et al., 2017; Rissanen et al., 2018), rendering the decryption of these interactions a critical necessity with respect to future therapeutic approaches. In fact, therapeutic strategies via microglial replacement are increasingly appreciated as novel avenues in restoring the integrity of white matter and proper CNS functioning per se (Beckmann et al., 2018; Han et al., 2019; Lloyd et al., 2017; Waisman et al., 2015). However, whether treatment of mice at young age may reduce the pace of the underyling $C_{n} p^{-1}$ phenotype, possibly by (partially) restoring healthy microglial functions, remains to be addressed by systematic characterization and profiling of repopulated microglia. Noteworthy, a study by Elmore et al. (2015) using PLX3397, a less selective variant of the CSF1-R inhibitor (Dagher et al., 2015), reported newly repopulated microglia to exhibit an inflammatory profile and functioning comparable to resident microglia in healthy adult mice. In another study using PLX5622, Elmore et al. (2018) further reported microglial repopulation, 4 weeks after cessation, to improve age-related cognitive impairments in healthy mice, along with re-juvenated microglial properties. While these findings do not reflect the microglial properties of young adult $C n p^{-1-}$ mice, they certainly are of interest with regard to our observation of sustainable reduction even 4 weeks after cessation of treatment.

Importantly, a prolonged treatment at a more progressed state of the underlying $\mathrm{Cnp}^{-1}$ phenotype, i.e. 27 to 35 weeks, successfully alleviated the catatonia-like phenotype, along with a reduction of neuroinflammatory processes, namely microgliosis $\left(\mathrm{IBA} 1^{+}\right.$), phagocytic microglial activities $\left(\mathrm{CD}^{+} 8^{+}\right)$, axonal spheroids $\left(\mathrm{APP}^{+}\right)$, astrogliosis $\left(\mathrm{GFAP}^{+}\right)$and infiltrating $\mathrm{T}$ lymphocytes $\left(\mathrm{CD}^{+}\right)$. Collectively, these findings strongly suggest a crucial involvement of abberant microglial behavior and neuroinflammation, induced by white matter pertubations, with respect to the catatonia-like phenotype of $\mathrm{Cnp}^{-1-}$ mice. Moreover, microglia-targeted pharmaceutical treatment via PLX5622 successfully prevents the onset of the phenotype upon early application and further alleviates catatonia at a progressed disease state. Yet, the timepoint of application as well as the impact of any CSF1-R inhibitor on other target cells have to be considered with respect to the outcome of the treatment (Han et al., 2019; Nelson \& Lenz, 2017). To this end, we observed a reduced efficacy of microglial depletion upon progressed neurodegenerative state of $\mathrm{Cnp}^{-1-}$ mice. Furthermore, we cannot exclude involvement of other cell types equally affected by PLX5622, such as infiltrating T lymphocytes, astroglia or OPCs $\left(\operatorname{PDGFR} \alpha^{+}\right)$. Further studies are required to systematically delineate the functionality of individual glial cell lines both in the development of the here reported catatonia-like phenotype 


\section{Conclusions \& Future Perspectives}

and its reduction by CSF1-R inhibition. Irrespectively, to my knowledge, the here reported mouse model is the first construct-valid animal model of catatonia suggesting key involvement of disrupted white matter integrity as well as secondary neuroinflammation/abberant microglial activities. These insights provide new opportunities to investigate the underyling mechanisms of the psychomotor syndrome of catatonia, ultimately aiming to develop a more selective and targeted therapeutic strategy (Rogers et al., 2019). It is, however, important to bear in mind that clinical presentation of catatonia in patients is heterogeneous in both etiology and symptomatology. Moreover, catatonia has a high comorbidity with various medical conditions, rendering neuroinflammation of subcortical white matter, as reported here, as one among various possible causes. Noteworthy, we confirmed the importance of disrupted white matter integrity in the here reported model, since we did not obrserve catatonic signs in WT mice upon intraperitoneal injection of lipopolysaccharide (LPS), a well-established method to induce chronic neuroinflammation and-degeneration (Qin et al., 2007). Application of LPS further did not amplify catatonic signs observed in $P h p^{-/ y}$ mice, suggesting a crucial involvement of disrupted underyling white matter integrity in the observed catatonia-like phenotype in our model.

\section{Catatonic signs upon perturbed white matter integrity are accompanied by executive dysfunction in mice and man}

Previously, Hagemeyer et al. (2012) and Poggi et al. (2016) reported age-dependent elevated signs of neuroinflammation particularly in frontal brain regions of $\mathrm{Cnp}^{+/-}$and $\mathrm{Mbp} \mathrm{p}^{+/}$, suggesting a key involvement of inherent structures with respect to the observed phenotypes. Additionally, elevated signs of neuroinflammation were also reported in schizophrenic carriers of the CNP SNP rs2070106-AA. In the framework of the here reported Project I, we further confirmed these findings, since we noted a progression of neuroinflammation into the cingulate cortex of $\mathrm{Cnp}^{-1}$ at a progressed disease state. Taking into account that the catatonic phenotype was observed as early as 8 weeks of age, i.e. prior to any known motor dysfunctions of $\mathrm{Cnp}^{-1-}$ mice at progressed age (Lappe-Siefke et al., 2003), we hypothesized that the catatonia-like phenotype is likely a reflection of transient executive dysfunction rather than any underlying motor impairments. This assumption is further supported by the fact that $C n p^{+/-}$mice exhibit a catatonia-depression syndrome upon aging, while motor function is not impaired (Hagemeyer et al., 2012). 


\section{Conclusions \& Future Perspectives}

Executive function is defined as a higher-order cognitive ability, particularly of the frontal lobes (Alvarez \& Emory, 2006; Otero \& Barker, 2014; Stuss \& Benson, 1984), comprising numerous functions such as selective attention, cognitive flexibility and problem solving (Diamond, 2013). The PFC has been reported to play a substantial part in proper and healthy execution of these functions (Chudasama, 2011; Yuan \& Raz, 2014). While a battery of behavioral experiments assessing distinct features of executive function in rodents are available (Heisler et al., 2015; Izquierdo et al., 2006; Sanchez-Roige et al., 2012), the majority of them come with unfavorable shortcomings such as multiple phase approaches or motivation via food or water deprivation, rendering such approaches time consuming, more vulnerable to unwanted confounding factors and increased stress via deprivation. Hence, the development of a novel, and importantly fast and reliable behavioral paradigm based on the innate behavior of mice was crucial to the assessment of executive function in our model. In a recent publication by Fernandez GarciaAgudo et al. (2019), we reported the Hurdle test as a respective novel test to assess executive function, while avoiding the above-mentioned shortcomings, by making use of the innate drive of mice to escape from a brightly lit open space to a shelter, i.e. walled and dimmed periphery. Application of the Hurdle test on $\mathrm{Cnp}^{-1}$ mice indeed revealed executive dysfunction. Importantly, we further reported catatonic signs and executive dysfunction to correlate in both mice and man. The latter was assessed by application of the Trail Making Test B (Periáñez et al., 2007) and the Luria Test (Chen et al., 1995), both of which cover distinct features of executive function. These findings not only indicate the relevance of executive function in the context of catatonia, but further allow for an improved assessment of the latter by combination of two robust behavioral paradigms in mice. Surprisingly, analogous early-on and delayed treatment of $\mathrm{Cnp}^{-1}$ with PLX5622, as conducted in Project I, did not reveal alleviation of executive impairments as assessed by the Hurdle test, suggesting involvement of additional underlying causes to the catatonia-related executive dysfunctions, other than neuroinflammation alone. Clearly, future studies are required to investigate the underlying mechanisms of the differential responsiveness of catatonic signs (Bar test) and executive dysfunction (Hurdle test) in the here reported mouse model of catatonia. Nevertheless, the studies conducted in the framework of the article by Fernandez Garcia-Agudo et al. (2019) confirmed the relevance of executive dysfunction in the etiology of the observed catatonia-like phenotype, induced by pertubations of white matter integrity and secondary neuroinflammation. 


\section{Conclusions \& Future Perspectives}

\section{Forebrain-specific loss of white matter integrity suffices to induce catatonia and related executive dysfuntion in mice}

Based on these findings, I employed a second project, in which we investigated and characterized mice with a $\mathrm{cKO}$ of $P / p 1$ in frontal brain structures, further aiming to elaborate the role of the latter in the framework of catatonia in our models. Experimental male $P h p^{f l o x / y *} E m x^{C r e}$ and female $P l p^{f b x / f b x *} E m x^{C r e}$ mice were generated in close collaboration with Prof. Klaus-Armin Nave, Ph.D., and PD Dr. Hauke B. Werner, essentially lacking PLP and its isoform DM20 in oligodendrocytes of the forebrain (Gorski et al., 2002). Functionality of the mouse model was confirmed by respective immunohistochemical analysis resulting in lack of PLP in the PFC, the hippocampal fimbria as well the CC, but not in untargeted control regions. Importantly, myelination per se was not affected, since IHC revealed no differences in MBP in the PFC. Moreover, neuropathological assessment revealed prominent neuroinflammatory and -degenerative features in the fimbria and $\mathrm{CC}$ of $\mathrm{cKO}$ mice, comprising degeneration $\left(\mathrm{APP}^{+}\right)$, microgliosis $\left(\mathrm{IBA}^{+}, \mathrm{MAC}^{+}\right)$and astrogliosis $\left(\mathrm{GFAP}^{+}\right)$, all of which are known from other Plp1 KO mouse models (Griffiths et al., 1998; Lüders et al., 2019; Lüders et al., 2017). Importantly, these observations were not made in the PFC, as expected. Collectively, these data confirm the targeted deletion of $P \not p 1$ in oligodendrocytes of the forebrain, resulting in the anticipated loss of myelin integrity along with secondary axonal degeneration and neuroinflammation.

The successful generation of this forebrain-specfic cKO renders the model an optimal tool to elaborate the importance of forebrain white matter integrity in catatonia and catatonia-related executive dysfunctions. In a recent publication by Gould et al. (2018), the authors reported distinct behavioral anomalies of Plp1 null mice, all of which preceded their known motor impairments (Griffiths et al., 1998). Interestingly, among those observations the authors reported impaired executive function assessed by another behavioral experiment, the Puzzle box (Ben Abdallah et al., 2011; Gould et al., 2018; O'Connor et al., 2014; Pease-Raissi \& Chan, 2018). Moreover, we observed catatonic signs via the Bar test in Plp1 null mice in the framework of my first project. Knowing that catatonic signs and executive dysfunction are correlated (Garcia-Agudo et al., 2019), I conducted a thorough behavioral characterization of the here reported newly generated cKO mice, particularly focusing on the former two domains. Behavioral assessment of male and female mice comprised a broad spectrum of domains, such as motor and sensory function, coordination, sensorimotor gating, social behavor, depressionlike behavior and cognitive abilities such as working memory, spatial memory and cognitive flexibility. In contrast to the diverse observations reported by Gould et al. (2018) on Plp1 null mice, $\mathrm{cKO}$ of $P \not p 1$ in the forebrain did not result in any of the previously reported phenotypes, 


\section{Conclusions \& Future Perspectives}

including motor and sensory performance, coordination, anxiety-like behavior or stereotypy. In fact, $\mathrm{cKO}$ mice of both genders remained largely unaffected, except a gender-dependent onset of catatonia along with executive dysfunction, assessed by two behavioral paradigms, namely the Hurdle test and the Puzzle box. The conduction of a subsequent second trial of the former, consistently revealed a reduced ratio [escape latency / number of crossed hurdles +1 ] in both genders, further reflecting an executive dysfunction upon initial exposure during the first trial. However, we report differences in the performance of male and female mice upon testing of the Hurdle test at 6 and 20 months, likely reflecting age- and gender-related effects in this behavioral paradigm. Neuropathological comparison of both genders, specifically describing the extent of axonopathy and neuroinflammation at these ages, would be worthwile to further investigate the underlying cause of the here reported differences. Importantly, as reported by Gould et al. (2018), development of these phenotypes occurred in the absence of any motor impairments known to develop in Plp1 null mice (Griffiths et al., 1998). In addition, overnight voluntary complex wheel running, a cortico-motor task relying on proper myelination (Gibson et al., 2014; McKenzie et al., 2014), in aged mice (23 months), further indicated altered executive function rather than motor impairments, since cKO mice of both genders exhibited reduced running times and distances, but not running velocities.

These findings further confirm a critical involvement of perturbed white matter integrity along with neuroinflammatory processes with respect to the catatonia-like phenotype observed in our mouse models. Moreover, by cKO of $\mathrm{Plp} 1$ specifically in forebrain structures, we report the latter to be essential to both catatonia and related executive dysfunctions. These findings are in accordance with previous reports on catatonia and impaired executive functions in patients suffering from altered white matter integrity particularly in the frontal lobes (Arora \& Praharaj, 2007; Northoff et al., 2004). Moreover, a higher-order fronto-parietal concept of catatonia has been reported by several neuroimaging studies, implying key involvements of the parietal cortex and the PFC (Hirjak et al., 2020). Upon usage of the here reported P/p1 cKO mice, we ultimately dissected the role of forebrain white matter integrity with regard to catatonia and executive function, the latter of which was reported upon constitutive lack of PLP (Gould et al., 2018), from known late-onset motor impairments reported in $P / p 1$ null mice (Griffiths et al., 1998). In conclusion, I report disrupted white matter integrity of of the forebrain, along with secondary neuroinflammation and axonopathy, to be essential to the etiology of a catatonia-like phenotype in our mouse models. While the myelin genes $C n p^{-1-}$ and $P / p 1$ clearly execute distinct tasks in the framework of proper myelination and healthy CNS functioning (Edgar \& Nave, 2009; Nave \& Werner, 2014), deficiency of either results in comparable neuropathological features, comprising altered white matter integrity and secondary neurodegeneration and -inflammation. 


\section{Conclusions \& Future Perspectives}

Although, pathology - caused by the individual lack of each gene - results in distinct spatiotemporal progression of such (Patzig et al., 2016), it is certainly interesting that combination of both have been reported to enhance these detrimental features (Lüders et al., 2017). While we do confirm neuroinflammation of subcortical white matter to be causative to catatonia and executive dysfunction in the here reported mouse model, further questions remain to be tackled. For instance, a novel approach by combination of $C n p^{-1}$ and $P / p 1$-deficient mice could be employed to investigate, whether the enhanced neuropathology translates into an enhanced behavioral phenotype as well. Moreover, the underlying mechanisms observed here in both animal models should be further investigated aiming to narrow down the precise alteration upon perturbed white matter integrity, which ultimately causes the here reported phenotype. It would be interesting to thereby elaborate whether both mouse models share other pathological features that result in the catatonia-like phenotype and further to which extent these features can be targeted by CSF1-R inhibition or microglial replacement strategies, both of which are increasingly appreciated as novel tools to tackle CNS disorders (Chitu et al., 2016; Han et al., 2019; Waisman et al., 2015). Noteworthy, altered white matter integrity has been compared to a prematurely aging brain in mice and primates (Hagemeyer et al., 2012; Peters \& Kemper, 2012). Hence, possibly respective processes, reflecting premature aging of the CNS per se, may contribute to the here reported executive dysfunctions, since we report the latter to specifically manifest upon aging in $\mathrm{P} / \mathrm{p} 1 \mathrm{cKO}$ mice of both genders.

Collectively, the here reported insights provide novel avenues to further investigate the underlying mechanisms of the psychomotor syndrome of catatonia (Rogers et al., 2019). However, I note that catatonia in patients is very heterogeneous in etiology and symptomatology along with a high comorbidity with various clinical conditions. Thus, the here reported model of neuroinflammation of subcortical white matter may not be the cause of catatonia in all catatonic patients. Nevertheless, our model clearly provides a novel and reliable animal model much needed in future research, ultimately aiming not only to expand our understanding of the psychomotor syndrome of catatonia but further to develop respective targeted and efficient therapeutic strategies. 
Chapter 5

\section{Bibliography}




\section{Bibliography}

Aggarwal, S., Snaidero, N., Pähler, G., Frey, S., Sánchez, P., Zweckstetter, M., et al. (2013). Myelin membrane assembly is driven by a phase transition of myelin basic proteins into a cohesive protein meshwork. PLoS biology, 11(6).

Ajami, B., Bennett, J. L., Krieger, C., Tetzlaff, W., \& Rossi, F. M. (2007). Local self-renewal can sustain CNS microglia maintenance and function throughout adult life. Nature neuroscience, 10(12), 1538-1543.

Alliot, F., Godin, I., \& Pessac, B. (1999). Microglia derive from progenitors, originating from the yolk sac, and which proliferate in the brain. Developmental Brain Research, 117(2), 145152.

Almkvist, O., Wahlund, L.-O., Andersson-Lundman, G., Basun, H., \& Bäckman, L. (1992). White-matter hyperintensity and neuropsychological functions in dementia and healthy aging. Archives of Neurology, 49(6), 626-632.

Alvarez, J. A., \& Emory, E. (2006). Executive function and the frontal lobes: a meta-analytic review. Neuropsychology review, 16(1), 17-42.

American Psychiatric Association. (2013). Diagnostic and statistical manual of mental disorders (DSM5®): American Psychiatric Pub.

Anderson, T., Schneider, A., Barrie, J., Klugmann, M., McCulloch, M., Kirkham, D., et al. (1998). Late-onset neurodegeneration in mice with increased dosage of the proteolipid protein gene. Journal of Comparative Neurology, 394(4), 506-519.

Appiani, F. J., \& Castro, G. S. (2018). Catatonia is not schizophrenia and it is treatable. Schizophrenia research, 200, 112-116.

Arora, M., \& Praharaj, S. K. (2007). Butterfly glioma of corpus callosum presenting as catatonia. The World Journal of Biological Psychiatry, 8(1), 54-55.

Asai, H., Ikezu, S., Tsunoda, S., Medalla, M., Luebke, J., Haydar, T., et al. (2015). Depletion of microglia and inhibition of exosome synthesis halt tau propagation. Nature neuroscience, 18(11), 1584.

Aston, C., Jiang, L., \& Sokolov, B. (2005). Transcriptional profiling reveals evidence for signaling and oligodendroglial abnormalities in the temporal cortex from patients with major depressive disorder. Molecular psychiatry, 10(3), 309-322.

Ayata, P., Badimon, A., Strasburger, H. J., Duff, M. K., Montgomery, S. E., Loh, Y.-H. E., et al. (2018). Epigenetic regulation of brain region-specific microglia clearance activity. Nature neuroscience, $21(8), 1049-1060$.

Bahro, M., Kämpf, C., \& Strnad, J. (1999). Catatonia under medication with risperidone in a 61year-old patient. Acta Psychiatrica Scandinavica, 99(3), 223-226.

Baraban, M., Mensch, S., \& Lyons, D. A. (2016). Adaptive myelination from fish to man. Brain research, 1641, 149-161.

Bauer, N. G., Richter-Landsberg, C., \& Ffrench-Constant, C. (2009). Role of the oligodendroglial cytoskeleton in differentiation and myelination. Glia, 57(16), 16911705.

Baumann, N., Turpin, J.-C., Lefevre, M., \& Colsch, B. (2002). Motor and psycho-cognitive clinical types in adult metachromatic leukodystrophy: genotype/phenotype relationships? Journal of Physiology-Paris, 96(3-4), 301-306.

Beach, S. R., Gomez-Bernal, F., Huffman, J. C., \& Fricchione, G. L. (2017). Alternative treatment strategies for catatonia: a systematic review. General hospital psychiatry, 48, 1-19.

Beckmann, N., Giorgetti, E., Neuhaus, A., Zurbruegg, S., Accart, N., Smith, P., et al. (2018). Brain region-specific enhancement of remyelination and prevention of demyelination by the CSF1R kinase inhibitor BLZ945. Acta neuropathologica communications, 6(1), 9. 
Begemann, M., Grube, S., Papiol, S., Malzahn, D., Krampe, H., Ribbe, K., et al. (2010). Modification of cognitive performance in schizophrenia by complexin 2 gene polymorphisms. Archives of general psychiatry, 67(9), 879-888.

Ben Abdallah, N. M.-B., Fuss, J., Trusel, M., Galsworthy, M. J., Bobsin, K., Colacicco, G., et al. (2011). The puzzle box as a simple and efficient behavioral test for exploring impairments of general cognition and executive functions in mouse models of schizophrenia. Experimental neurology, 227(1), 42-52.

Bengtsson, S. L., Nagy, Z., Skare, S., Forsman, L., Forssberg, H., \& Ullén, F. (2005). Extensive piano practicing has regionally specific effects on white matter development. Nature neuroscience, 8(9), 1148-1150.

Bianchin, M. M., Capella, H. M., Chaves, D. L., Steindel, M., Grisard, E. C., Ganev, G. G., et al. (2004). Nasu-Hakola disease (polycystic lipomembranous osteodysplasia with sclerosing leukoencephalopathy_PLOSL): a dementia associated with bone cystic lesions. From clinical to genetic and molecular aspects. Cellular and molecular neurobiology, 24(1), 1-24.

Bleuler, E. (1911). Dementia praecox: oder Gruppe der Schizophrenien: F. Deuticke.

Boggs, J. (2006). Myelin basic protein: a multifunctional protein. Cellular and Molecular Life Sciences CMLS, 63(17), 1945-1961.

Bowley, M. P., Cabral, H., Rosene, D. L., \& Peters, A. (2010). Age changes in myelinated nerve fibers of the cingulate bundle and corpus callosum in the rhesus monkey. Journal of Comparative Neurology, 518(15), 3046-3064.

Braun, P. E., Sandillon, F., Edwards, A., Matthieu, J.-M., \& Privat, A. (1988). Immunocytochemical localization by electron microscopy of 2'3'-cyclic nucleotide 3'phosphodiesterase in developing oligodendrocytes of normal and mutant brain. Journal of Neuroscience, 8(8), 3057-3066.

Bräunig, P., Krüger, S., Shugar, G., Höffler, J., \& Börner, I. (2000). The catatonia rating scale I-development, reliability, and use. Comprehensive Psychiatry, 41(2), 147-158.

Bunge, R. P. (1968). Glial cells and the central myelin sheath. Physiological reviews, 48(1), 197-251.

Bush, G., Fink, M., Petrides, G., Dowling, F., \& Francis, A. (1996a). Catatonia. I. Rating scale and standardized examination. Acta Psychiatrica Scandinavica, 93(2), 129-136.

Bush, G., Fink, M., Petrides, G., Dowling, F., \& Francis, A. (1996b). Catatonia. II. Treatment with lorazepam and electroconvulsive therapy. Acta Psychiatrica Scandinavica, 93(2), 137143.

Carpenter, P. A., Just, M. A., \& Reichle, E. D. (2000). Working memory and executive function: Evidence from neuroimaging. Current opinion in neurobiology, 10(2), 195-199.

Cartier, N., Lewis, C.-A., Zhang, R., \& Rossi, F. M. (2014). The role of microglia in human disease: therapeutic tool or target? Acta neuropathologica, 128(3), 363-380.

Cassidy, E., O'Brien, M., Osman, M., Finucane, J., \& O'Keane, V. (2001). Lethal catatonia responding to high-dose olanzapine therapy. Journal of Psychopharmacology, 15(4), 302-304.

Chan, R. C., Shum, D., Toulopoulou, T., \& Chen, E. Y. (2008). Assessment of executive functions: Review of instruments and identification of critical issues. Archives of clinical neuropsychology, 23(2), 201-216.

Chang, L., Munsaka, S. M., Kraft-Terry, S., \& Ernst, T. (2013). Magnetic resonance spectroscopy to assess neuroinflammation and neuropathic pain. Journal of neuroimmune pharmacology, 8(3), 576-593.

Chen, E. Y., Shapleske, J., Luque, R., McKenna, P. J., Hodges, J. R., Calloway, S. P., et al. (1995). The Cambridge Neurological Inventory: a clinical instrument for assessment of soft neurological signs in psychiatric patients. Psychiatry Research, 56(2), 183-204.

Chernoff, G. F. (1981). Shiverer: an autosomal recessive mutant mouse with myelin deficiency. Journal of Heredity, 72(2), 128-128. 
Chitu, V., Gokhan, S.., Nandi, S., Mehler, M. F., \& Stanley, E. R. (2016). Emerging roles for CSF-1 receptor and its ligands in the nervous system. Trends in neurosciences, 39(6), 378393.

Choi, D. K., Koppula, S., \& Suk, K. (2011). Inhibitors of microglial neurotoxicity: focus on natural products. Molecules, 16(2), 1021-1043.

Chudasama, Y. (2011). Animal models of prefrontal-executive function. Behavioral neuroscience, 125(3), 327.

Colonna, M., \& Butovsky, O. (2017). Microglia function in the central nervous system during health and neurodegeneration. Annual review of immunology, 35, 441-468.

Dagher, N. N., Najafi, A. R., Kayala, K. M. N., Elmore, M. R., White, T. E., Medeiros, R., et al. (2015). Colony-stimulating factor 1 receptor inhibition prevents microglial plaque association and improves cognition in 3xTg-AD mice. Journal of neuroinflammation, 12(1), 139.

Davalos, D., Grutzendler, J., Yang, G., Kim, J. V., Zuo, Y., Jung, S., et al. (2005). ATP mediates rapid microglial response to local brain injury in vivo. Nature neuroscience, 8(6), 752-758.

De Biase, L. M., Schuebel, K. E., Fusfeld, Z. H., Jair, K., Hawes, I. A., Cimbro, R., et al. (2017). Local cues establish and maintain region-specific phenotypes of basal ganglia microglia. Neuron, 95(2), 341-356. e346.

de Monasterio-Schrader, P., Jahn, O., Tenzer, S., Wichert, S. P., Patzig, J., \& Werner, H. B. (2012). Systematic approaches to central nervous system myelin. Cellular and molecular life sciences, 69(17), 2879-2894.

de Monasterio-Schrader, P., Patzig, J., Möbius, W., Barrette, B., Wagner, T. L., Kusch, K., et al. (2013). Uncoupling of neuroinflammation from axonal degeneration in mice lacking the myelin protein tetraspanin-2. Glia, 61(11), 1832-1847.

Dean, D. C., Hurley, S. A., Kecskemeti, S. R., O’Grady, J. P., Canda, C., Davenport-Sis, N. J., et al. (2017). Association of amyloid pathology with myelin alteration in preclinical Alzheimer disease. JAMA neurology, 74(1), 41-49.

Dere, E., Dahm, L., Lu, D., Hammerschmidt, K., Ju, A., Tantra, M., et al. (2014). Heterozygous ambra1 deficiency in mice: a genetic trait with autism-like behavior restricted to the female gender. Frontiers in behavioral neuroscience, 8, 181.

Dere, E., Ronnenberg, A., Tampe, B., Arinrad, S., Schmidt, M., Zeisberg, E., et al. (2018). Cognitive, emotional and social phenotyping of mice in an observer-independent setting. Neurobiology of learning and memory, 150, 136-150.

Diamond, A. (2013). Executive functions. Annual review of psychology, 64, 135-168.

Doorn, K. J., Brevé, J. J., Drukarch, B., Boddeke, H. W., Huitinga, I., Lucassen, P. J., et al. (2015). Brain region-specific gene expression profiles in freshly isolated rat microglia. Frontiers in cellular neuroscience, 9, 84.

Doorn, K. J., Moors, T., Drukarch, B., van de Berg, W. D., Lucassen, P. J., \& van Dam, A.-M. (2014). Microglial phenotypes and toll-like receptor 2 in the substantia nigra and hippocampus of incidental Lewy body disease cases and Parkinson's disease patients. Acta neuropathologica communications, 2(1), 90.

Du, L., Zhang, Y., Chen, Y., Zhu, J., Yang, Y., \& Zhang, H.-L. (2017). Role of microglia in neurological disorders and their potentials as a therapeutic target. Molecular neurobiology, 54(10), 7567-7584.

Duncan, I., Hammang, J., \& Trapp, B. (1987). Abnormal compact myelin in the myelin-deficient rat: absence of proteolipid protein correlates with a defect in the intraperiod line. Proceedings of the National Academy of Sciences, 84(17), 6287-6291.

Edgar, J. M., McLaughlin, M., Yool, D., Zhang, S.-C., Fowler, J. H., Montague, P., et al. (2004). Oligodendroglial modulation of fast axonal transport in a mouse model of hereditary spastic paraplegia. The Journal of cell biology, 166(1), 121-131.

Edgar, J. M., \& Nave, K.-A. (2009). The role of CNS glia in preserving axon function. Current opinion in neurobiology, 19(5), 498-504. 
Edgar, N., \& Sibille, E. (2012). A putative functional role for oligodendrocytes in mood regulation. Translational psychiatry, 2(5), e109-e109.

El Khoury, J. (2010). Neurodegeneration and the neuroimmune system. Nature medicine, 16(12), 1369-1370.

Elmore, M. R., Hohsfield, L. A., Kramár, E. A., Soreq, L., Lee, R. J., Pham, S. T., et al. (2018). Replacement of microglia in the aged brain reverses cognitive, synaptic, and neuronal deficits in mice. Aging cell, 17(6), e12832.

Elmore, M. R., Lee, R. J., West, B. L., \& Green, K. N. (2015). Characterizing newly repopulated microglia in the adult mouse: impacts on animal behavior, cell morphology, and neuroinflammation. PloS one, 10(4).

Elmore, M. R., Najafi, A. R., Koike, M. A., Dagher, N. N., Spangenberg, E. E., Rice, R. A., et al. (2014). Colony-stimulating factor 1 receptor signaling is necessary for microglia viability, unmasking a microglia progenitor cell in the adult brain. Neuron, 82(2), 380397.

Eyo, U. B., \& Wu, L.-J. (2013). Bidirectional microglia-neuron communication in the healthy brain. Neural plasticity, 2013.

Fields, R. D. (2008). White matter in learning, cognition and psychiatric disorders. Trends in neurosciences, 31(7), 361-370.

Filley, C. M., \& Fields, R. D. (2016). White matter and cognition: making the connection. Journal of neurophysiology, 116(5), 2093-2104.

Flores, A. I., Narayanan, S. P., Morse, E. N., Shick, H. E., Yin, X., Kidd, G., et al. (2008). Constitutively active Akt induces enhanced myelination in the CNS. Journal of Neuroscience, 28(28), 7174-7183.

Frick, L. R., Williams, K., \& Pittenger, C. (2013). Microglial dysregulation in psychiatric disease. Clinical and developmental immunology, 2013.

Frühbeis, C., Fröhlich, D., Kuo, W. P., Amphornrat, J., Thilemann, S., Saab, A. S., et al. (2013). Neurotransmitter-triggered transfer of exosomes mediates oligodendrocyte-neuron communication. PLoS biology, 11(7).

Fünfschilling, U., Supplie, L. M., Mahad, D., Boretius, S., Saab, A. S., Edgar, J., et al. (2012). Glycolytic oligodendrocytes maintain myelin and long-term axonal integrity. Nature, 485(7399), 517-521.

Garbern, J. Y., Yool, D. A., Moore, G. J., Wilds, I. B., Faulk, M. W., Klugmann, M., et al. (2002). Patients lacking the major CNS myelin protein, proteolipid protein 1, develop lengthdependent axonal degeneration in the absence of demyelination and inflammation. Brain, 125(3), 551-561.

Garcia-Agudo, L. F., Janova, H., Sendler, L. E., Arinrad, S., Steixner, A. A., Hassouna, I., et al. (2019). Genetically induced brain inflammation by Cnp deletion transiently benefits from microglia depletion. The FASEB Journal, 33(7), 8634-8647.

Garde, E., Mortensen, E. L., Rostrup, E., \& Paulson, O. (2005). Decline in intelligence is associated with progression in white matter hyperintensity volume. Journal of Neurology, Neurosurgery \& Psychiatry, 76(9), 1289-1291.

Gibson, E. M., Purger, D., Mount, C. W., Goldstein, A. K., Lin, G. L., Wood, L. S., et al. (2014). Neuronal activity promotes oligodendrogenesis and adaptive myelination in the mammalian brain. science, 344(6183), 1252304.

Ginhoux, F., Greter, M., Leboeuf, M., Nandi, S., See, P., Gokhan, S., et al. (2010). Fate mapping analysis reveals that adult microglia derive from primitive macrophages. science, 330(6005), 841-845.

Girish, K., \& Gill, N. S. (2003). Electroconvulsive therapy in lorazepam non-responsive catatonia. Indian journal of psychiatry, 45(1), 21.

Glezer, I., Simard, A., \& Rivest, S. (2007). Neuroprotective role of the innate immune system by microglia. Neuroscience, 147(4), 867-883. 
Gomez-Nicola, D., \& Perry, V. H. (2015). Microglial dynamics and role in the healthy and diseased brain: a paradigm of functional plasticity. The Neuroscientist, 21(2), 169-184.

Gorski, J. A., Talley, T., Qiu, M., Puelles, L., Rubenstein, J. L., \& Jones, K. R. (2002). Cortical excitatory neurons and glia, but not GABAergic neurons, are produced in the Emx1expressing lineage. Journal of Neuroscience, 22(15), 6309-6314.

Gosselin, D., Link, V. M., Romanoski, C. E., Fonseca, G. J., Eichenfield, D. Z., Spann, N. J., et al. (2014). Environment drives selection and function of enhancers controlling tissuespecific macrophage identities. Cell, 159(6), 1327-1340.

Gosselin, D., Skola, D., Coufal, N. G., Holtman, I. R., Schlachetzki, J. C., Sajti, E., et al. (2017). An environment-dependent transcriptional network specifies human microglia identity. science, 356(6344), eaal3222.

Gould, E. A., Busquet, N., Shepherd, D., Dietz, R. M., Herson, P. S., de Souza, F. M. S., et al. (2018). Mild myelin disruption elicits early alteration in behavior and proliferation in the subventricular zone. Elife, 7, e34783.

Grabert, K., Michoel, T., Karavolos, M. H., Clohisey, S., Baillie, J. K., Stevens, M. P., et al. (2016). Microglial brain region- dependent diversity and selective regional sensitivities to aging. Nature neuroscience, 19(3), 504.

Graeber, M. B., Li, W., \& Rodriguez, M. L. (2011). Role of microglia in CNS inflammation. FEBS letters, 585(23), 3798-3805.

Graham, K. T., \& Carroll, B. T. (2001). Dopamine in catatonia. Journal of clinical psychopharmacology, 21(6), 624-625.

Gravel, M., Peterson, J., Yong, V. W., Kottis, V., Trapp, B., \& Braun, P. E. (1996). Overexpression of 2', 3'-cyclic nucleotide 3'-phosphodiesterase in transgenic mice alters oligodendrocyte development and produces aberrant myelination. Molecular and Cellular Neuroscience, 7(6), 453-466.

Greter, M., Lelios, I., Pelczar, P., Hoeffel, G., Price, J., Leboeuf, M., et al. (2012). Stroma-derived interleukin-34 controls the development and maintenance of langerhans cells and the maintenance of microglia. Immunity, 37(6), 1050-1060.

Griffiths, I., Klugmann, M., Anderson, T., Yool, D., Thomson, C., Schwab, M. H., et al. (1998). Axonal swellings and degeneration in mice lacking the major proteolipid of myelin. science, 280(5369), 1610-1613.

Groh, J., Klein, D., Berve, K., West, B. L., \& Martini, R. (2019). Targeting microglia attenuates neuroinflammation-related neural damage in mice carrying human PLP1 mutations. Glia, 67(2), 277-290.

Gutiérrez, R., Boison, D., Heinemann, U., \& Stoffel, W. (1995). Decompaction of CNS myelin leads to a reduction of the conduction velocity of action potentials in optic nerve. Neuroscience letters, 195(2), 93-96.

Hagemeyer, N., Goebbels, S., Papiol, S., Kästner, A., Hofer, S., Begemann, M., et al. (2012). A myelin gene causative of a catatonia-depression syndrome upon aging. EMBO molecular medicine, 4(6), 528-539.

Hagemeyer, N., Hanft, K.-M., Akriditou, M.-A., Unger, N., Park, E. S., Stanley, E. R., et al. (2017). Microglia contribute to normal myelinogenesis and to oligodendrocyte progenitor maintenance during adulthood. Acta neuropathologica, 134(3), 441-458.

Hakak, Y., Walker, J. R., Li, C., Wong, W. H., Davis, K. L., Buxbaum, J. D., et al. (2001). Genome-wide expression analysis reveals dysregulation of myelination-related genes in chronic schizophrenia. Proceedings of the National Academy of Sciences, 98(8), 4746-4751.

Hammer, C., Stepniak, B., Schneider, A., Papiol, S., Tantra, M., Begemann, M., et al. (2014). Neuropsychiatric disease relevance of circulating anti-NMDA receptor autoantibodies depends on blood-brain barrier integrity. Molecular psychiatry, 19(10), 1143-1149.

Hammerschmidt, K., Radyushkin, K., Ehrenreich, H., \& Fischer, J. (2012). The structure and usage of female and male mouse ultrasonic vocalizations reveal only minor differences. PloS one, 7(7), e41133. 
Han, J., Zhu, K., Zhang, X. M., \& Harris, R. A. (2019). Enforced microglial depletion and repopulation as a promising strategy for the treatment of neurological disorders. Glia, 67(2), 217-231.

Haroutunian, V., Katsel, P., Roussos, P., Davis, K., Altshuler, L., \& Bartzokis, G. (2014). Myelination, oligodendrocytes, and serious mental illness. Glia, 62(11), 1856-1877.

Heisler, J. M., Morales, J., Donegan, J. J., Jett, J. D., Redus, L., \& O'connor, J. C. (2015). The attentional set shifting task: a measure of cognitive flexibility in mice. JoVE (Journal of Visualized Experiments)(96), e51944.

Hickman, S. E., Kingery, N. D., Ohsumi, T. K., Borowsky, M. L., Wang, L.-c., Means, T. K., et al. (2013). The microglial sensome revealed by direct RNA sequencing. Nature neuroscience, 16(12), 1896.

Hildebrand, C., Remahl, S., Persson, H., \& Bjartmar, C. (1993). Myelinated nerve fibres in the CNS. Progress in neurobiology, 40(3), 319-384.

Hirjak, D., Kubera, K. M., Wolf, R. C., \& Northoff, G. (2020). Going back to Kahlbaum's psychomotor (and GABAergic) origins: is catatonia more than just a motor and dopaminergic syndrome? Schizophrenia bulletin, 46(2), 272-285.

Hirrlinger, J., \& Nave, K. A. (2014). Adapting brain metabolism to myelination and long-range signal transduction. Glia, 62(11), 1749-1761.

Holmes, S. E., Hinz, R., Conen, S., Gregory, C. J., Matthews, J. C., Anton-Rodriguez, J. M., et al. (2018). Elevated translocator protein in anterior cingulate in major depression and a role for inflammation in suicidal thinking: a positron emission tomography study. Biological psychiatry, 83(1), 61-69.

Hong, S., Beja-Glasser, V. F., Nfonoyim, B. M., Frouin, A., Li, S., Ramakrishnan, S., et al. (2016). Complement and microglia mediate early synapse loss in Alzheimer mouse models. science, 352(6286), 712-716.

Huang, Y., Xu, Z., Xiong, S., Sun, F., Qin, G., Hu, G., et al. (2018). Repopulated microglia are solely derived from the proliferation of residual microglia after acute depletion. Nature neuroscience, 21(4), 530-540.

Hughes, E. G., Kang, S. H., Fukaya, M., \& Bergles, D. E. (2013). Oligodendrocyte progenitors balance growth with self-repulsion to achieve homeostasis in the adult brain. Nature neuroscience, 16(6), 668.

Inoue, K. (2019). Pelizaeus-Merzbacher Disease: Molecular and Cellular Pathologies and Associated Phenotypes Myelin (pp. 201-216): Springer.

Irvine, K., \& Blakemore, W. (2008). Remyelination protects axons from demyelinationassociated axon degeneration. Brain, 131(6), 1464-1477.

Iseki, K., Ikeda, A., Kihara, T., Kawamoto, Y., Mezaki, T., Hanakawa, T., et al. (2009). Impairment of the cortical GABAergic inhibitory system in catatonic stupor: a case report with neuroimaging. Epileptic Disorders, 11(2), 126-131.

Izquierdo, A., Wiedholz, L. M., Millstein, R. A., Yang, R. J., Bussey, T. J., Saksida, L. M., et al. (2006). Genetic and dopaminergic modulation of reversal learning in a touchscreenbased operant procedure for mice. Behavioural brain research, 171(2), 181-188.

Jahn, O., Tenzer, S., \& Werner, H. B. (2009). Myelin proteomics: molecular anatomy of an insulating sheath. Molecular neurobiology, 40(1), 55-72.

Jamain, S., Radyushkin, K., Hammerschmidt, K., Granon, S., Boretius, S., Varoqueaux, F., et al. (2008). Reduced social interaction and ultrasonic communication in a mouse model of monogenic heritable autism. Proceedings of the National Academy of Sciences, 105(5), 17101715.

Janova, H., Arinrad, S., Balmuth, E., Mitjans, M., Hertel, J., Habes, M., et al. (2018). Microglia ablation alleviates myelin-associated catatonic signs in mice. The Journal of clinical investigation, 128(2), 734-745. 
Jockusch, W. J., Speidel, D., Sigler, A., Sørensen, J. B., Varoqueaux, F., Rhee, J.-S., et al. (2007). CAPS-1 and CAPS-2 are essential synaptic vesicle priming proteins. Cell, 131(4), 796808.

Jung, M., Sommer, I., Schachner, M., \& Nave, K. A. (1996). Monoclonal antibody O10 defines a conformationally sensitive cell-surface epitope of proteolipid protein (PLP): evidence that PLP misfolding underlies dysmyelination in mutant mice. J Neurosci, 16(24), 79207929.

Kahlbaum, K. (1874). Die Katatonie oder das Spannungsirresein, eine klinische form psychischer krankheit, 1st Hirschwald.

Kahlbaum, K. (2007). The clinico-diagnostic perspective in psychopathology'. History of psychiatry, 18(2), 233-245.

Kassmann, C. M., Lappe-Siefke, C., Baes, M., Brügger, B., Mildner, A., Werner, H. B., et al. (2007). Axonal loss and neuroinflammation caused by peroxisome-deficient oligodendrocytes. Nature genetics, 39(8), 969-976.

Keller, T. A., \& Just, M. A. (2009). Altering cortical connectivity: remediation-induced changes in the white matter of poor readers. Neuron, 64(5), 624-631.

Kettenmann, H., Kirchhoff, F., \& Verkhratsky, A. (2013). Microglia: new roles for the synaptic stripper. Neuron, 77(1), 10-18.

Kierdorf, K., Erny, D., Goldmann, T., Sander, V., Schulz, C., Perdiguero, E. G., et al. (2013). Microglia emerge from erythromyeloid precursors via Pu. 1-and Irf8-dependent pathways. Nature neuroscience, 16(3), 273.

Kierdorf, K., \& Prinz, M. (2017). Microglia in steady state. The Journal of clinical investigation, 127(9), 3201-3209.

Kirby, B. B., Takada, N., Latimer, A. J., Shin, J., Carney, T. J., Kelsh, R. N., et al. (2006). In vivo time-lapse imaging shows dynamic oligodendrocyte progenitor behavior during zebrafish development. Nature neuroscience, 9(12), 1506-1511.

Klein, D., Patzko, A., Schreiber, D., van Hauwermeiren, A., Baier, M., Groh, J., et al. (2015). Targeting the colony stimulating factor 1 receptor alleviates two forms of CharcotMarie-Tooth disease in mice. Brain, 138(11), 3193-3205.

Klugmann, M., Schwab, M. H., Pühlhofer, A., Schneider, A., Zimmermann, F., Griffiths, I. R., et al. (1997). Assembly of CNS myelin in the absence of proteolipid protein. Neuron, 18(1), 59-70.

Kondo, Y., Kinoshita, M., Fukushima, K., Yoshida, K., \& Ikeda, S.-i. (2013). Early involvement of the corpus callosum in a patient with hereditary diffuse leukoencephalopathy with spheroids carrying the de novo K793T mutation of CSF1R. Internal Medicine, 52(4), 503506.

Konno, T., Tada, M., Tada, M., Koyama, A., Nozaki, H., Harigaya, Y., et al. (2014). Haploinsufficiency of CSF-1R and clinicopathologic characterization in patients with HDLS. Neurology, 82(2), 139-148.

Kraepelin, E. (1899). Psychiatrie. Ein Lehrbuch für Studierende und Ärzte. Barth, 6. Aufl. Leiprig.

Kuhn, S., Gritti, L., Crooks, D., \& Dombrowski, Y. (2019). Oligodendrocytes in Development, Myelin Generation and Beyond. Cells, 8(11), 1424.

Kusch, K., Uecker, M., Liepold, T., Mobius, W., Hoffmann, C., Neumann, H., et al. (2017). Partial Immunoblotting of 2D-Gels: A Novel Method to Identify Post-Translationally Modified Proteins Exemplified for the Myelin Acetylome. Proteomes, 5(1).

Kuschiski, K., \& Hornykiewicz, O. (1972). Morphine catalepsy in the rat: relation to striatal dopamine metabolism. European journal of pharmacology, 19(1), 119-122.

Lampron, A., Larochelle, A., Laflamme, N., Préfontaine, P., Plante, M.-M., Sánchez, M. G., et al. (2015). Inefficient clearance of myelin debris by microglia impairs remyelinating processes. Journal of Experimental Medicine, 212(4), 481-495. 
Lappe-Siefke, C., Goebbels, S., Gravel, M., Nicksch, E., Lee, J., Braun, P. E., et al. (2003). Disruption of Cnp1 uncouples oligodendroglial functions in axonal support and myelination. Nature genetics, 33(3), 366-374.

Lasiene, J., Matsui, A., Sawa, Y., Wong, F., \& Horner, P. J. (2009). Age-related myelin dynamics revealed by increased oligodendrogenesis and short internodes. Aging cell, 8(2), 201-213.

Lawson, L. J., Perry, V. H., Dri, P., \& Gordon, S. (1990). Heterogeneity in the distribution and morphology of microglia in the normal adult mouse brain. Neuroscience, 39(1), 151-170.

Lee, A. S., Azmitia, E. C., \& Whitaker-Azmitia, P. M. (2017). Developmental microglial priming in postmortem autism spectrum disorder temporal cortex. Brain, behavior, and immunity, 62, 193-202.

Lee, J., Gravel, M., Zhang, R., Thibault, P., \& Braun, P. E. (2005). Process outgrowth in oligodendrocytes is mediated by $\mathrm{CNP}$, a novel microtubule assembly myelin protein. The Journal of cell biology, 170(4), 661-673.

Lee, Y., Morrison, B. M., Li, Y., Lengacher, S., Farah, M. H., Hoffman, P. N., et al. (2012). Oligodendroglia metabolically support axons and contribute to neurodegeneration. Nature, 487(7408), 443-448.

Liu, J., Dietz, K., DeLoyht, J. M., Pedre, X., Kelkar, D., Kaur, J., et al. (2012). Impaired adult myelination in the prefrontal cortex of socially isolated mice. Nature neuroscience, 15(12), 1621-1623.

Lloyd, A. F., Davies, C. L., \& Miron, V. E. (2017). Microglia: origins, homeostasis, and roles in myelin repair. Current opinion in neurobiology, 47, 113-120.

Lüders, K. A., Nessler, S., Kusch, K., Patzig, J., Jung, R. B., Möbius, W., et al. (2019). Maintenance of high proteolipid protein level in adult central nervous system myelin is required to preserve the integrity of myelin and axons. Glia, 67(4), 634-649.

Lüders, K. A., Patzig, J., Simons, M., Nave, K. A., \& Werner, H. B. (2017). Genetic dissection of oligodendroglial and neuronal Plp1 function in a novel mouse model of spastic paraplegia type 2. Glia, 65(11), 1762-1776.

Lund, C., Mortimer, A., Rogers, D., \& McKenna, P. (1991). Motor, Volitional and Behavioural Disorders in Schizophrenia: 1: Assessment Using the Modified Rogers Scale. The British Journal of Psychiatry, 158(3), 323-327.

Madigand, J., Lebain, P., Callery, G., \& Dollfus, S. (2016). Catatonic syndrome: from detection to therapy. L'Encéphale, 42(4), 340-345.

Makinodan, M., Rosen, K. M., Ito, S., \& Corfas, G. (2012). A critical period for social experience-dependent oligodendrocyte maturation and myelination. science, 337(6100), 1357-1360.

Markham-Abedi, C., McNeely, C., \& de Leon, J. (2007). A case report with ziprasidone-induced catatonic symptoms. The Journal of neuropsychiatry and clinical neurosciences, 19(4), 476-477.

Marshall, G. P. I., Deleyrolle, L. P., Reynolds, B. A., Steindler, D. A., \& Laywell, E. D. (2014). Microglia from neurogenic and non-neurogenic regions display differential proliferative potential and neuroblast support. Frontiers in cellular neuroscience, 8, 180.

Martényi, F., Metcalfe, S., Schausberger, B., \& Dossenbach, M. (2001). An efficacy analysis of olanzapine treatment data in schizophrenia patients with catatonic signs and symptoms. The Journal of clinical psychiatry, 62, 25-27.

Mastroeni, D., Nolz, J., Sekar, S., Delvaux, E., Serrano, G., Cuyugan, L., et al. (2018). Lasercaptured microglia in the Alzheimer's and Parkinson's brain reveal unique regional expression profiles and suggest a potential role for hepatitis B in the Alzheimer's brain. Neurobiology of aging, 63, 12-21.

Matcovitch-Natan, O., Winter, D. R., Giladi, A., Aguilar, S. V., Spinrad, A., Sarrazin, S., et al. (2016). Microglia development follows a stepwise program to regulate brain homeostasis. science, 353(6301), aad8670. 
McGeer, P. L., Itagaki, S., Tago, H., \& McGeer, E. G. (1987). Reactive microglia in patients with senile dementia of the Alzheimer type are positive for the histocompatibility glycoprotein HLA-DR. Neuroscience letters, 79(1-2), 195-200.

McKenna, P., Lund, C., Mortimer, A., \& Biggins, C. (1991). Motor, Volitional and Behavioural Disorders in Schizophrenia: 2: The 'Conflict of Paradigms' Hypothesis. The British Journal of Psychiatry, 158(3), 328-336.

McKenzie, I. A., Ohayon, D., Li, H., De Faria, J. P., Emery, B., Tohyama, K., et al. (2014). Motor skill learning requires active central myelination. science, 346(6207), 318-322.

Mei, F., Lehmann-Horn, K., Shen, Y.-A. A., Rankin, K. A., Stebbins, K. J., Lorrain, D. S., et al. (2016). Accelerated remyelination during inflammatory demyelination prevents axonal loss and improves functional recovery. Elife, 5, e18246.

Michalski, J.-P., \& Kothary, R. (2015). Oligodendrocytes in a nutshell. Frontiers in cellular neuroscience, 9, 340.

Milner, R. J., Lai, C., Nave, K.-A., Lenoir, D., Ogata, J., \& Sutcliffe, J. G. (1985). Nucleotide sequences of two mRNAs for rat brain myelin proteolipid protein. Cell, 42(3), 931-939.

Miron, V. E. (2017). Microglia-driven regulation of oligodendrocyte lineage cells, myelination, and remyelination. Journal of leukocyte biology, 101(5), 1103-1108.

Mitkus, S. N., Hyde, T. M., Vakkalanka, R., Kolachana, B., Weinberger, D. R., Kleinman, J. E., et al. (2008). Expression of oligodendrocyte-associated genes in dorsolateral prefrontal cortex of patients with schizophrenia. Schizophrenia research, 98(1-3), 129-138.

Möbius, W., Nave, K.-A., \& Werner, H. B. (2016). Electron microscopy of myelin: structure preservation by high-pressure freezing. Brain research, 1641, 92-100.

Möbius, W., Patzig, J., Nave, K.-A., \& Werner, H. B. (2008). Phylogeny of proteolipid proteins: divergence, constraints, and the evolution of novel functions in myelination and neuroprotection. Neuron glia biology, 4(2), 111-127.

Monji, A., Kato, T. A., Mizoguchi, Y., Horikawa, H., Seki, Y., Kasai, M., et al. (2013). Neuroinflammation in schizophrenia especially focused on the role of microglia. Progress in Neuro-Psychopharmacology and Biological Psychiatry, 42, 115-121.

Moore, S., Meschkat, M., Ruhwedel, T., Tzvetanova, I. D., Trevisiol, A., Battefeld, A., et al. (2019). A role of oligodendrocytes in information processing independent of conduction velocity. bioRxiv, 736975.

Morgan, J. T., Chana, G., Pardo, C. A., Achim, C., Semendeferi, K., Buckwalter, J., et al. (2010). Microglial activation and increased microglial density observed in the dorsolateral prefrontal cortex in autism. Biological psychiatry, 68(4), 368-376.

Mosconi, L., Mistur, R., Switalski, R., Tsui, W. H., Glodzik, L., Li, Y., et al. (2009). FDG-PET changes in brain glucose metabolism from normal cognition to pathologically verified Alzheimer's disease. European journal of nuclear medicine and molecular imaging, 36(5), 811 822.

Mosher, K. I., \& Wyss-Coray, T. (2015). Go with your gut: microbiota meet microglia. Nature neuroscience, 18(7), 930-931.

Mount, C. W., \& Monje, M. (2017). Wrapped to adapt: experience-dependent myelination. Neuron, 95(4), 743-756.

Mukherjee, C., Kling, T., Russo, B., Miebach, K., Kess, E., Schifferer, M., et al. (2020). Oligodendrocytes Provide Antioxidant Defense Function for Neurons by Secreting Ferritin Heavy Chain. Cell Metabolism.

Muneer, A. (2016). The neurobiology of bipolar disorder: an integrated approach. Chonnam medical journal, 52(1), 18-37.

Murase, S.-i., \& Hayashi, Y. (1998). Expression pattern and neurotrophic role of the c-fms proto-oncogene M-CSF receptor in rodent Purkinje cells. Journal of Neuroscience, 18(24), 10481-10492. 
Naaldijk, Y. M., Bittencourt, M. C., Sack, U., \& Ulrich, H. (2016). Kinins and microglial responses in bipolar disorder: a neuroinflammation hypothesis. Biological chemistry, 397(4), 283-296.

Nandi, S., Gokhan, S., Dai, X.-M., Wei, S., Enikolopov, G., Lin, H., et al. (2012). The CSF-1 receptor ligands IL-34 and CSF-1 exhibit distinct developmental brain expression patterns and regulate neural progenitor cell maintenance and maturation. Developmental biology, 367(2), 100-113.

Narayanan, S. P., Flores, A. I., Wang, F., \& Macklin, W. B. (2009). Akt signals through the mammalian target of rapamycin pathway to regulate CNS myelination. Journal of Neuroscience, 29(21), 6860-6870.

Nave, K.-A. (2010). Myelination and support of axonal integrity by glia. Nature, 468(7321), 244252.

Nave, K.-A., \& Boespflug-Tanguy, O. (1996). X-linked developmental defects of myelination: from mouse mutants to human genetic diseases. The Neuroscientist, 2(1), 33-43.

Nave, K.-A., \& Ehrenreich, H. (2014). Myelination and oligodendrocyte functions in psychiatric diseases. JAMA psychiatry, 71(5), 582-584.

Nave, K.-A., Lai, C., Bloom, F. E., \& Milner, R. J. (1987). Splice site selection in the proteolipid protein (PLP) gene transcript and primary structure of the DM-20 protein of central nervous system myelin. Proceedings of the National Academy of Sciences, 84(16), 5665-5669.

Nave, K.-A., \& Werner, H. B. (2014). Myelination of the nervous system: mechanisms and functions. Annual review of cell and developmental biology, 30, 503-533.

Nawaz, S., Schweitzer, J., Jahn, O., \& Werner, H. B. (2013). Molecular evolution of myelin basic protein, an abundant structural myelin component. Glia, 61(8), 1364-1377.

Nelson, L. H., \& Lenz, K. M. (2017). Microglia depletion in early life programs persistent changes in social, mood-related, and locomotor behavior in male and female rats. Behavioural brain research, 316, 279-293.

Netrakanti, P. R., Cooper, B. H., Dere, E., Poggi, G., Winkler, D., Brose, N., et al. (2015). Fast cerebellar reflex circuitry requires synaptic vesicle priming by munc13-3. The Cerebellum, 14(3), 264-283.

Nikodemova, M., Kimyon, R. S., De, I., Small, A. L., Collier, L. S., \& Watters, J. J. (2015). Microglial numbers attain adult levels after undergoing a rapid decrease in cell number in the third postnatal week. Journal of neuroimmunology, 278, 280-288.

Nimmerjahn, A., Kirchhoff, F., \& Helmchen, F. (2005). Resting microglial cells are highly dynamic surveillants of brain parenchyma in vivo. science, 308(5726), 1314-1318.

Nissen, J. C., Thompson, K. K., West, B. L., \& Tsirka, S. E. (2018). Csf1R inhibition attenuates experimental autoimmune encephalomyelitis and promotes recovery. Experimental neurology, 307, 24-36.

Northoff, G. (2000). Brain imaging in catatonia: current findings and a pathophysiologic model. CNS spectrums, 5(7), 34-46.

Northoff, G., Braus, D., Sartorius, A., Khoram-Sefat, D., Russ, M., Eckert, J., et al. (1999). Reduced activation and altered laterality in two neuroleptic-naive catatonic patients during a motor task in functional MRI. Psychological medicine, 29(4), 997-1002.

Northoff, G., Koch, A., Wenke, J., Eckert, J., Böker, H., Pflug, B., et al. (1999). Catatonia as a psychomotor syndrome: a rating scale and extrapyramidal motor symptoms. Movement disorders: official journal of the Movement Disorder Society, 14(3), 404-416.

Northoff, G., Kötter, R., Baumgart, F., Danos, P., Boeker, H., Kaulisch, T., et al. (2004). Orbitofrontal cortical dysfunction in akinetic catatonia: a functional magnetic resonance imaging study during negative emotional stimulation. Schizophrenia bulletin, 30(2), 405427.

Northoff, G., Wenke, J., Demisch, L., Eckert, J., Gille, B., \& Pflug, B. (1995). Catatonia: shortterm response to lorazepam and dopaminergic metabolism. Psychopharmacology, 122(2), 182-186. 
Numata, S., Kato, O., Misawa, H., Kanai, T., Kasahara, T., \& Ohmori, T. (2001). Treatment of catatonia with olanzapine. Pharmacopsychiatry, 34, 25-26.

O'Connor, A. M., Burton, T. J., Leamey, C. A., \& Sawatari, A. (2014). The use of the puzzle box as a means of assessing the efficacy of environmental enrichment. JoVE (Journal of Visualized Experiments)(94), e52225.

Olah, M., Amor, S., Brouwer, N., Vinet, J., Eggen, B., Biber, K., et al. (2012). Identification of a microglia phenotype supportive of remyelination. Glia, 60(2), 306-321.

Oldham, M. (2019). A diversified theory of catatonia. The lancet. Psychiatry, 6(7), 554-555.

Otero, T. M., \& Barker, L. A. (2014). The frontal lobes and executive functioning Handbook of executive functioning (pp. 29-44): Springer.

Paloneva, J., Autti, T., Raininko, R., Partanen, J., Salonen, O., Puranen, M., et al. (2001). CNS manifestations of Nasu-Hakola disease: a frontal dementia with bone cysts. Neurology, 56(11), 1552-1558.

Patzig, J., Kusch, K., Fledrich, R., Eichel, M. A., Lüders, K. A., Möbius, W., et al. (2016). Proteolipid protein modulates preservation of peripheral axons and premature death when myelin protein zero is lacking. Glia, 64(1), 155-174.

Pease-Raissi, S. E., \& Chan, J. R. (2018). Micro (glial)-managing executive function: white matter inflammation drives catatonia. The Journal of clinical investigation, 128(2), 564-566.

Perdiguero, E. G., Klapproth, K., Schulz, C., Busch, K., Azzoni, E., Crozet, L., et al. (2015). Tissue-resident macrophages originate from yolk-sac-derived erythro-myeloid progenitors. Nature, 518(7540), 547-551.

Periáñez, J., Rios-Lago, M., Rodríguez-Sánchez, J., Adrover-Roig, D., Sánchez-Cubillo, I., Crespo-Facorro, B., et al. (2007). Trail Making Test in traumatic brain injury, schizophrenia, and normal ageing: Sample comparisons and normative data. Archives of clinical neuropsychology, 22(4), 433-447.

Perry, V. H., \& Holmes, C. (2014). Microglial priming in neurodegenerative disease. Nature Reviews Neurology, 10(4), 217-224.

Peters, A. (2002). The effects of normal aging on myelin and nerve fibers: a review. Journal of neurocytology, 31(8-9), 581-593.

Peters, A., \& Kemper, T. (2012). A review of the structural alterations in the cerebral hemispheres of the aging rhesus monkey. Neurobiology of aging, 33(10), 2357-2372.

Petit, B., Giraudet, F., Béchon, C., Bardin, L., Avan, P., Boespflug-Tanguy, O., et al. (2014). Mice with a deletion of the major central myelin protein exhibit hypersensitivity to noxious thermal stimuli: involvement of central sensitization. Neurobiology of disease, 65, 55-68.

Poggi, G., Boretius, S., Möbius, W., Moschny, N., Baudewig, J., Ruhwedel, T., et al. (2016). Cortical network dysfunction caused by a subtle defect of myelination. Glia, 64(11), 2025-2040.

Purger, D., Gibson, E. M., \& Monje, M. (2016). Myelin plasticity in the central nervous system. Neuropharmacology, 110, 563-573.

Qin, L., Wu, X., Block, M. L., Liu, Y., Breese, G. R., Hong, J. S., et al. (2007). Systemic LPS causes chronic neuroinflammation and progressive neurodegeneration. Glia, 55(5), 453462.

Rademakers, R., Baker, M., Nicholson, A. M., Rutherford, N. J., Finch, N., Soto-Ortolaza, A., et al. (2012). Mutations in the colony stimulating factor 1 receptor (CSF1R) gene cause hereditary diffuse leukoencephalopathy with spheroids. Nature genetics, 44(2), 200.

Ransohoff, R. M., \& El Khoury, J. (2016). Microglia in health and disease. Cold Spring Harbor perspectives in biology, 8(1), a020560.

Rasband, M. N., Tayler, J., Kaga, Y., Yang, Y., Lappe-Siefke, C., Nave, K. A., et al. (2005). CNP is required for maintenance of axon-glia interactions at nodes of Ranvier in the CNS. Glia, 50(1), 86-90. 
Readhead, C., \& Hood, L. (1990). The dysmyelinating mouse mutations shiverer (shi) and myelin deficient (shi mld). Behavior genetics, 20(2), 213-234.

Readhead, C., Popko, B., Takahashi, N., Shine, H. D., Saavedra, R. A., Sidman, R. L., et al. (1987). Expression of a myelin basic protein gene in transgenic shiverer mice: correction of the dysmyelinating phenotype. Cell, 48(4), 703-712.

Ribbe, K., Friedrichs, H., Begemann, M., Grube, S., Papiol, S., Kästner, A., et al. (2010). The cross-sectional GRAS sample: a comprehensive phenotypical data collection of schizophrenic patients. BMC psychiatry, 10(1), 91.

Richter, A., Grimm, S., \& Northoff, G. (2010). Lorazepam modulates orbitofrontal signal changes during emotional processing in catatonia. Human Psychopharmacology: Clinical and Experimental, 25(1), 55-62.

Rinholm, J. E., Hamilton, N. B., Kessaris, N., Richardson, W. D., Bergersen, L. H., \& Attwell, D. (2011). Regulation of oligodendrocyte development and myelination by glucose and lactate. Journal of Neuroscience, 31(2), 538-548.

Rissanen, E., Tuisku, J., Vahlberg, T., Sucksdorff, M., Paavilainen, T., Parkkola, R., et al. (2018). Microglial activation, white matter tract damage, and disability in MS. NeurologyNeuroimmunology Neuroinflammation, 5(3).

Ritzel, R. M., Patel, A. R., Pan, S., Crapser, J., Hammond, M., Jellison, E., et al. (2015). Age-and location-related changes in microglial function. Neurobiology of aging, 36(6), 2153-2163.

Roach, A., Boylan, K., Horvath, S., Prusiner, S. B., \& Hood, L. E. (1983). Characterization of cloned cDNA representing rat myelin basic protein: absence of expression in brain of shiverer mutant mice. Cell, 34(3), 799-806.

Rogers, J. P., Pollak, T. A., Blackman, G., \& David, A. S. (2019). Catatonia and the immune system: a review. The Lancet Psychiatry.

Rosenbluth, J., Nave, K. A., Mierzwa, A., \& Schiff, R. (2006). Subtle myelin defects in PLP-null mice. Glia, 54(3), 172-182.

Ross, B. D., Bluml, S., Cowan, R., Danielsen, E., Farrow, N., \& Gruetter, R. (1997). In vivo magnetic resonance spectroscopy of human brain: the biophysical basis of dementia. Biophysical chemistry, 68(1-3), 161-172.

Roussos, P., \& Haroutunian, V. (2014). Schizophrenia: susceptibility genes and oligodendroglial and myelin related abnormalities. Frontiers in cellular neuroscience, $8,5$.

Saab, A. S., Tzvetanova, I. D., \& Nave, K.-A. (2013). The role of myelin and oligodendrocytes in axonal energy metabolism. Current opinion in neurobiology, 23(6), 1065-1072.

Saab, A. S., Tzvetavona, I. D., Trevisiol, A., Baltan, S., Dibaj, P., Kusch, K., et al. (2016). Oligodendroglial NMDA receptors regulate glucose import and axonal energy metabolism. Neuron, 91(1), 119-132.

Saher, G., Rudolphi, F., Corthals, K., Ruhwedel, T., Schmidt, K.-F., Löwel, S., et al. (2012). Therapy of Pelizaeus-Merzbacher disease in mice by feeding a cholesterol-enriched diet. Nature medicine, 18(7), 1130.

Salter, M. W., \& Stevens, B. (2017). Microglia emerge as central players in brain disease. Nature medicine, 23(9), 1018.

Sampaio-Baptista, C., \& Johansen-Berg, H. (2017). White matter plasticity in the adult brain. Neuron, 96(6), 1239-1251.

Sampaio-Baptista, C., Khrapitchev, A. A., Foxley, S., Schlagheck, T., Scholz, J., Jbabdi, S., et al. (2013). Motor skill learning induces changes in white matter microstructure and myelination. Journal of Neuroscience, 33(50), 19499-19503.

Sanchez-Roige, S., Peña-Oliver, Y., \& Stephens, D. N. (2012). Measuring impulsivity in mice: the five-choice serial reaction time task. Psychopharmacology, 219(2), 253-270.

Saugier-Veber, P., Munnich, A., Bonneau, D., Rozet, J.-M., Le Merrer, M., Gil, R., et al. (1994). $\mathrm{X}$-linked spastic paraplegia and Pelizaeus-Merzbacher disease are allelic disorders at the proteolipid protein locus. Nature genetics, 6(3), 257. 
Schafer, D. P., Lehrman, E. K., Kautzman, A. G., Koyama, R., Mardinly, A. R., Yamasaki, R., et al. (2012). Microglia sculpt postnatal neural circuits in an activity and complementdependent manner. Neuron, 74(4), 691-705.

Schain, A. J., Hill, R. A., \& Grutzendler, J. (2014). Label-free in vivo imaging of myelinated axons in health and disease with spectral confocal reflectance microscopy. Nature medicine, 20(4), 443.

Schardt, A., Brinkmann, B. G., Mitkovski, M., Sereda, M. W., Werner, H. B., \& Nave, K.-A. (2009). The SNARE protein SNAP-29 interacts with the GTPase Rab3A: implications for membrane trafficking in myelinating glia. Journal of Neuroscience Research, 87, 34653479.

Scherer, S. S. (2015). Blocking bad. Brain, 138(11), 3132-3133.

Scherer, S. S., Braun, P. E., Grinspan, J., Collarini, E., Wang, D.-Y., \& Kamholz, J. (1994). Differential regulation of the 2', 3'-cyclic nucleotide 3'-phosphodiesterase gene during oligodendrocyte development. Neuron, 12(6), 1363-1375.

Scheuerecker, J., Ufer, S., Käpernick, M., Wiesmann, M., Brückmann, H., Kraft, E., et al. (2009). Cerebral network deficits in post-acute catatonic schizophrenic patients measured by fMRI. Journal of psychiatric research, 43(6), 607-614.

Seethalakshmi, R., Dhavale, S., Suggu, K., \& Dewan, M. (2008). Catatonic syndrome: importance of detection and treatment with lorazepam. Annals of Clinical Psychiatry, 20(1), 5-8.

Sereda, M., Griffiths, I., Pühlhofer, A., Stewart, H., Rossner, M. J., Zimmermann, F., et al. (1996). A transgenic rat model of Charcot-Marie-Tooth disease. Neuron, 16(5), 10491060.

Sereda, M. W., \& Nave, K.-A. (2006). Animal models of Charcot-Marie-Tooth disease type 1A. Neuromolecular medicine, 8(1-2), 205-215.

Shen, Z., Li, X., Bao, X., \& Wang, R. (2017). Microglia-targeted stem cell therapies for Alzheimer disease: A preclinical data review. Journal of neuroscience research, 95(12), 24202429.

Sierra, A., Gottfried-Blackmore, A. C., McEwen, B. S., \& Bulloch, K. (2007). Microglia derived from aging mice exhibit an altered inflammatory profile. Glia, 55(4), 412-424.

Simons, M., \& Nave, K.-A. (2016). Oligodendrocytes: myelination and axonal support. Cold Spring Harborperspectives in biology, 8(1), a020479.

Snaidero, N., Möbius, W., Czopka, T., Hekking, L. H., Mathisen, C., Verkleij, D., et al. (2014). Myelin membrane wrapping of CNS axons by PI (3, 4, 5) P3-dependent polarized growth at the inner tongue. Cell, 156(1-2), 277-290.

Snaidero, N., Velte, C., Myllykoski, M., Raasakka, A., Ignatev, A., Werner, H. B., et al. (2017). Antagonistic functions of MBP and CNP establish cytosolic channels in CNS myelin. Cell reports, 18(2), 314-323.

Sosna, J., Philipp, S., Albay, R., Reyes-Ruiz, J. M., Baglietto-Vargas, D., LaFerla, F. M., et al. (2018). Early long-term administration of the CSF1R inhibitor PLX3397 ablates microglia and reduces accumulation of intraneuronal amyloid, neuritic plaque deposition and pre-fibrillar oligomers in 5XFAD mouse model of Alzheimer's disease. Molecular neurodegeneration, 13(1), 11.

Spittau, B. (2017). Aging microglia—phenotypes, functions and implications for age-related neurodegenerative diseases. Frontiers in aging neuroscience, 9, 194.

Srivastava, R. K., Bulte, J. W., Walczak, P., \& Janowski, M. (2018). Migratory potential of transplanted glial progenitors as critical factor for successful translation of glia replacement therapy: The gap between mice and men. Glia, 66(5), 907-919.

Stassart, R. M., Möbius, W., Nave, K.-A., \& Edgar, J. M. (2018). The axon-myelin unit in development and degenerative disease. Frontiers in neuroscience, 12, 467.

Steyer, A. M., Ruhwedel, T., Nardis, C., Werner, H. B., Nave, K.-A., \& Möbius, W. (2020). Pathology of myelinated axons in the PLP-deficient mouse model of spastic paraplegia 
type 2 revealed by volume imaging using focused ion beam-scanning electron microscopy. Journal of structural biology, 210(2), 107492.

Streit, W. J., Conde, J. R., Fendrick, S. E., Flanary, B. E., \& Mariani, C. L. (2005). Role of microglia in the central nervous system's immune response. Neurological research, 27(7), 685-691.

Streit, W. J., Mrak, R. E., \& Griffin, W. S. T. (2004). Microglia and neuroinflammation: a pathological perspective. Journal of neuroinflammation, 1(1), 14.

Sturrock, R. R. (1976). Changes in neuroglia and myelination in the white matter of aging mice. Journal of Gerontology, 31(5), 513-522.

Stuss, D. T., \& Benson, D. F. (1984). Neuropsychological studies of the frontal lobes. Psychological bulletin, 95(1), 3.

Subramaniam, S. R., \& Federoff, H. J. (2017). Targeting microglial activation states as a therapeutic avenue in Parkinson's disease. Frontiers in aging neuroscience, 9, 176.

Sullivan, E. V., Adalsteinsson, E., Hedehus, M., Ju, C., Moseley, M., Lim, K. O., et al. (2001). Equivalent disruption of regional white matter microstructure in ageing healthy men and women. Neuroreport, 12(1), 99-104.

Sundal, C., Van Gerpen, J. A., Nicholson, A. M., Wider, C., Shuster, E. A., Aasly, J., et al. (2012). MRI characteristics and scoring in HDLS due to CSF1R gene mutations. Neurology, 79(6), 566-574.

Tay, T. L., Béchade, C., D’Andrea, I., St-Pierre, M.-K., Henry, M. S., Roumier, A., et al. (2018). Microglia gone rogue: impacts on psychiatric disorders across the lifespan. Frontiers in molecular neuroscience, 10, 421.

Tkachev, D., Mimmack, M. L., Ryan, M. M., Wayland, M., Freeman, T., Jones, P. B., et al. (2003). Oligodendrocyte dysfunction in schizophrenia and bipolar disorder. The Lancet, 362(9386), 798-805.

Tomassy, G. S., Berger, D. R., Chen, H.-H., Kasthuri, N., Hayworth, K. J., Vercelli, A., et al. (2014). Distinct profiles of myelin distribution along single axons of pyramidal neurons in the neocortex. science, 344(6181), 319-324.

Trapp, B., Moench, T., Pulley, M., Barbosa, E., Tennekoon, G., \& Griffin, J. (1987). Spatial segregation of mRNA encoding myelin-specific proteins. Proceedings of the National Academy of Sciences, 84(21), 7773-7777.

Trapp, B. D., Nishiyama, A., Cheng, D., \& Macklin, W. (1997). Differentiation and death of premyelinating oligodendrocytes in developing rodent brain. The Journal of cell biology, 137(2), 459-468.

Trevisiol, A., Kusch, K., Steyer, A. M., Gregor, I., Nardis, C., Winkler, U., et al. (2020). Structural myelin defects are associated with low axonal ATP levels but rapid recovery from energy deprivation in a mouse model of spastic paraplegia. PLoS biology, 18(11), e3000943.

Trevisiol, A., Saab, A. S., Winkler, U., Marx, G., Imamura, H., Möbius, W., et al. (2017). Monitoring ATP dynamics in electrically active white matter tracts. Elife, 6, e24241.

Ungvari, G., Leung, C., Wong, M., \& Lau, J. (1994). Benzodiazepines in the treatment of catatonic syndrome. Acta Psychiatrica Scandinavica, 89(4), 285-288.

Ungvari, G. S., Chiu, H. F., Chow, L. Y., Lau, B. S., \& Tang, W. K. (1999). Lorazepam for chronic catatonia: a randomized, double-blind, placebo-controlled cross-over study. Psychopharmacology, 142(4), 393-398.

Valevski, A., Loebl, T., Keren, T., Bodinger, L., \& Weizman, A. (2001). Response of catatonia to risperidone: two case reports. Clinical neuropharmacology, 24(4), 228-231.

Van Den Eede, F., Van Hecke, J., Van Dalfsen, A., Van den Bossche, B., Cosyns, P., \& Sabbe, B. G. (2005). The use of atypical antipsychotics in the treatment of catatonia. European Psychiatry, 20(5-6), 422-429.

Van der Heijden, F., Tuinier, S., Arts, N., Hoogendoorn, M., Kahn, R., \& Verhoeven, W. (2005). Catatonia: disappeared or under-diagnosed? Psychopathology, 38(1), 3-8. 
Varvel, N. H., Grathwohl, S. A., Baumann, F., Liebig, C., Bosch, A., Brawek, B., et al. (2012). Microglial repopulation model reveals a robust homeostatic process for replacing CNS myeloid cells. Proceedings of the National Academy of Sciences, 109(44), 18150-18155.

Völzke, H., Alte, D., Schmidt, C. O., Radke, D., Lorbeer, R., Friedrich, N., et al. (2011). Cohort profile: the study of health in Pomerania. International journal of epidemiology, 40(2), 294307.

Waisman, A., Ginhoux, F., Greter, M., \& Bruttger, J. (2015). Homeostasis of microglia in the adult brain: review of novel microglia depletion systems. Trends in immunology, 36(10), 625-636.

Walther, S., Schäppi, L., Federspiel, A., Bohlhalter, S., Wiest, R., Strik, W., et al. (2017). Restingstate hyperperfusion of the supplementary motor area in catatonia. Schizophrenia bulletin, 43(5), 972-981.

Walther, S., Stegmayer, K., Wilson, J. E., \& Heckers, S. (2019). Structure and neural mechanisms of catatonia. The Lancet Psychiatry.

Wang, F., Ren, S.-Y., Chen, J.-F., Liu, K., Li, R.-X., Li, Z.-F., et al. (2020). Myelin degeneration and diminished myelin renewal contribute to age-related deficits in memory. Nature Neuroscience, 23(4), 481-486.

Wang, L., Winnewisser, J., Federle, C., Jessberger, G., Nave, K.-A., Werner, H. B., et al. (2017). Epitope-specific tolerance modes differentially specify susceptibility to proteolipid protein-induced experimental autoimmune encephalomyelitis. Frontiers in immunology, 8, 1511.

Wang, Q., Liu, Y., \& Zhou, J. (2015). Neuroinflammation in Parkinson's disease and its potential as therapeutic target. Translational Neurodegeneration, 4(1), 19.

Wang, Y., Szretter, K. J., Vermi, W., Gilfillan, S., Rossini, C., Cella, M., et al. (2012). IL-34 is a tissue-restricted ligand of CSF1R required for the development of Langerhans cells and microglia. Nature immunology, 13(8), 753.

Wang, Y. Q., Berezovska, O., \& Fedoroff, S. (1999). Expression of colony stimulating factor-1 receptor (CSF-1R) by CNS neurons in mice. Journal of neuroscience research, 57(5), 616-632.

Wei, S., Nandi, S., Chitu, V., Yeung, Y. G., Yu, W., Huang, M., et al. (2010). Functional overlap but differential expression of CSF-1 and IL-34 in their CSF-1 receptor-mediated regulation of myeloid cells. Journal of leukocyte biology, 88(3), 495-505.

Werner, H. B., Krämer-Albers, E. M., Strenzke, N., Saher, G., Tenzer, S., Ohno-Iwashita, Y., et al. (2013). A critical role for the cholesterol-associated proteolipids PLP and M6B in myelination of the central nervous system. Glia, 61(4), 567-586.

Werner, H. B., Kuhlmann, K., Shen, S., Uecker, M., Schardt, A., Dimova, K., et al. (2007). Proteolipid protein is required for transport of sirtuin 2 into CNS myelin. Journal of Neuroscience, 27(29), 7717-7730.

Wes, P. D., Holtman, I. R., Boddeke, E. W., Möller, T., \& Eggen, B. J. (2016). Next generation transcriptomics and genomics elucidate biological complexity of microglia in health and disease. Glia, 64(2), 197-213.

Wierzba-Bobrowicz, T., Lewandowska, E., Lechowicz, W., Stępień, T., \& Pasennik, E. (2005). Quantitative analysis of activated microglia, ramified and damage of processes in the frontal and temporal lobes of chronic schizophrenics. Folia Neuropathologica, 43(2).

Winkler, D., Daher, F., Wüstefeld, L., Hammerschmidt, K., Poggi, G., Seelbach, A., et al. (2018). Hypersocial behavior and biological redundancy in mice with reduced expression of PSD95 or PSD93. Behavioural brain research, 352, 35-45.

Winkler, E. A., Nishida, Y., Sagare, A. P., Rege, S. V., Bell, R. D., Perlmutter, D., et al. (2015). GLUT1 reductions exacerbate Alzheimer's disease vasculo-neuronal dysfunction and degeneration. Nature neuroscience, 18(4), 521. 


\section{Bibliography}

Wlodarczyk, A., Holtman, I. R., Krueger, M., Yogev, N., Bruttger, J., Khorooshi, R., et al. (2017). A novel microglial subset plays a key role in myelinogenesis in developing brain. The EMBO journal, 36(22), 3292-3308.

Wohleb, E. S., Terwilliger, R., Duman, C. H., \& Duman, R. S. (2018). Stress-induced neuronal colony stimulating factor 1 provokes microglia-mediated neuronal remodeling and depressive-like behavior. Biological psychiatry, 83(1), 38-49.

World Health Organization. (2004). ICD-10: international statistical classification of diseases and related health problems: tenth revision.

Xue, J., Schmidt, S. V., Sander, J., Draffehn, A., Krebs, W., Quester, I., et al. (2014). Transcriptome-based network analysis reveals a spectrum model of human macrophage activation. Immunity, 40(2), 274-288.

Yin, X., Peterson, J., Gravel, M., Braun, P., \& Trapp, B. (1997). CNP overexpression induces aberrant oligodendrocyte membranes and inhibits MBP accumulation and myelin compaction. Journal of neuroscience research, 50(2), 238-247.

Yool, D., Klugmann, M., McLaughlin, M., Vouyiouklis, D., Dimou, L., Barrie, J., et al. (2001). Myelin proteolipid proteins promote the interaction of oligodendrocytes and axons. Journal of neuroscience research, 63(2), 151-164.

Young, K. M., Psachoulia, K., Tripathi, R. B., Dunn, S.-J., Cossell, L., Attwell, D., et al. (2013). Oligodendrocyte dynamics in the healthy adult CNS: evidence for myelin remodeling. Neuron, 77(5), 873-885.

Yu, W.-P., Collarini, E. J., Pringle, N. P., \& Richardson, W. D. (1994). Embryonic expression of myelin genes: evidence for a focal source of oligodendrocyte precursors in the ventricular zone of the neural tube. Neuron, 12(6), 1353-1362.

Yuan, P., \& Raz, N. (2014). Prefrontal cortex and executive functions in healthy adults: a metaanalysis of structural neuroimaging studies. Neuroscience \& Biobehavioral Reviews, 42, $180-$ 192.

Zhou, B., Zhu, Z., Ransom, B. R., \& Tong, X. (2021). Oligodendrocyte lineage cells and depression. Molecular psychiatry, 26(1), 103-117. 


\section{Chapter 6}

\section{Appendices}




\title{
List of abbreviations
}

\author{
$A D$ Alzheimer's Disease \\ Ambra1 Autophagy and beclin 1 regulator 1 \\ ApoE Apolipoprotein-E \\ APP Amyloid precursor protein \\ ASD Autism Spectrum Disorder \\ ATP Adenosine Triphophate \\ $B B B$ Blood-brain barrier \\ Caps1 Calcium-dependent secretion activator 1 \\ CC Corpus callosum \\ CD3/68 Cluster of differentiation 3/68 \\ cKO Conditional knockout \\ CMT Charcot-Marie-Tooth Disease \\ CNP/Cnp 2',3'-cyclic nucleotide 3'-phosphodiesterase \\ CNS Central Nervous System \\ $C R W$ Complex running wheels \\ CSF1/CSF1-R Colony-stimulating factor $1 /$ receptor \\ Ctrl Control \\ CX3CR1 C-X3-C Motif Chemokine Receptor 1 \\ $d B$ Decibel \\ DSM5 Diagnostic and Statistical Manual of Mental Disorders 5 \\ DTI Diffusion Tensor Imaging \\ ECT Electroconvulsive Therapy \\ Emx1 Empty spiracles homebox 1 \\ ER Endoplasmatic reticulum \\ $G A B A \quad$ Gamma-aminobutoric acid \\ GFAP Glial fibrillary acidic protein \\ GWAS Genome-wide association study \\ HDLS Hereditary Diffuse Leukoencephalopathy with Spheroids \\ HRP Horseradish peroxidase \\ IBA1 Ionized calcium-binding adapter molecule 1 \\ ICD10 International Statistical Classification of Disease 10 \\ Il-34 Interleukin 34 \\ ITI Inter-trial-interval
}




\section{Appendix I}

LPS Lipopolysaccharide

MAC3 Lysosomal-associated membrane protein 2

MBP/Mbp Myelin basic protein

MCTs Monocarboxylate transporters

MDD Major Depression Disorder

MRI/MRS Magnetic Resonance Imaging/Spectroscopy

$m R N A$ Messenger Ribonucleic Acid

MS Multiple Sclerosis

NCRS Northoff Catatonia Rating Scale

NHD Nasu-Hakola Disease

Nlgn4 Neuroligin-4

NMDAR $\quad N$-methyl-D-aspartate receptor

OPCs Oligodendrocyte precursor cells

$P D$ Parkinson's Disease

PFC Prefrontal cortex

PLP/Ph Proteolipid protein

PLX5622 Plexxikon 5622

PMD Pelizaeus-Merzbacher Disease

PSD93/95 Postsynaptic density protein 93/95

PVDF Polyvinylidene difluoride

RFID Radio-frequency identification

$r p m$ Rotations per minute

SEM Standard error of mean

SNP Single nucleotide polymorphism

SPG2 Spastic paraplegia type 2

SVZ Subventricular zone

TBST Tris-buffered saline and Tween 20

WMH White matter hyperintensities

WT Wildtype 


\section{Peer-reviewed co-authorship publications}

I

Dere, E., Ronnenberg, A., Tampe, B., Arinrad, S., Schmidt, M., Zeisberg, E., \& Ehrenreich, H. (2018). Cognitive, emotional and social phenotyping of mice in an observer-independent setting. Neurobiology of learning and memory, 150, 136-150.

Personal contributions: I contributed to the preparation and the analysis of behavioral data of male and female WT mice (Figures 1-3).

\section{II}

Garcia-Agudo, L.F.*, Janova, H*., Sendler, L.E.*, Arinrad, S., Steixner, A.A., Hassouna, I., Balmuth, E., Ronnenberg, A., Schopf, N., van der Flier, F.J. , Begemann, M., Martens, H., Weber, M.S., Boretius, S., Nave, K.A. and Ehrenreich, H. (2019). Genetically induced brain inflammation by Cnp deletion transiently benefits from microglia depletion. The FASEB Journal , 33(7), 8634-8647.

Personal contributions: I carried out all behavioral experiments shown in Figures $1 \mathrm{~A}-\mathrm{C}$ and $\mathrm{E}$ - M, with assistance from Anja Ronnenberg in execution of the Hurdle test. Furthermore, I analyzed all Bar and Hurdle test data depicted and/or used in Figures 1C and F- M, while Agnes Stexiner used some of these datasets to prepare Figures 1C, K and M. I also analyzed all data depicted in Figures 1N - V and prepared all graphs, including all before-mentioned graphs with the exception of Figures 1C, K and M.

\section{III}

Wakhloo, D.*, Scharkowski, F.*, Curto, Y.*, Butt, U.J., Bansal, V., Steixner-Kumar, A.A., Wüstefeld, L., Rajput A., Arinrad, S., Zillmann, M.R., Seelbach, A., Hassouna, I., Schneider, K., Ibrahim., A.Q., Werner., H.B., Martens, H., Miskowiak, K., Wojcik, S.M., Bonn, S., Nacher, J., Nave, K.A. and Ehrenreich, H. (2020). Functional hypoxia drives neuroplasticity and neurogenesis via brain erythropoietin. Nature communications, 11(1), 1-12.

Personal contributions: I contributed to all CRW experimental setups and the respective analyses of behavioral data shown in Figures 4A - C, F- H and J. Additionally, I contributed to the overnight CRW experimental procedures and subsequent tissue collection for analysis of neuronal activity via labeling of cFos-positive neurons within the cornu ammonis 1 region of the hippocampus (Figures 4M-P). 


\section{Appendix II}

\section{IV}

Butt, U.J.*, Steixner-Kumar, A.A.*, Depp, C*, Sun, T., Hassouna, I., Wüstefeld, L., Arinrad, S., Zillmann, M., Schopf, N., Garcia-Agudo, L.F., Bode, U., Ronnenberg, A., Goebbels, S., Bonn,

S., Katschinski, D.M., Nave, K.A. and Ehrenreich, H. (2021). Hippocampal neurons respond to brain activity with functional hypoxia. Molecular Psychiatry, 26:1790-1807.

Personal contributions: I contributed to the acquisition and processing of behavioral data assessed in the CRW experiments (Figure 5). Moreover, I contributed to the extraction of hippocampal brain tissue from mice exposed to previous normoxic or hypoxic conditions or voluntary CRW activity. This tissue was used to label cell activity states via cFos labeling as well as expression levels of distinct genes within the hippocampi (Figure 5).

\section{V}

Wilke, J.B.H.*, Hindermann, M.*, Moussavi, A., Butt, U.J., Dadarwal, R., Berghoff, S.A., Sarcheshmeh, A.K., Ronnenberg, A., Zihsler, S., Arinrad, S., Hardeland, R., Seidel, J., Lühder, F., Nave, K.A, Boretius, S. and Ehrenreich, H. (2021). Inducing sterile pyramidal neuronal death in mice to model distinct aspects of gray matter encephalitis. Acta Neuropathologica Communications, 9(1), 1-19.

Personal contributions: I contributed to the organization, acquisition and processing of all behavioral data (Figures 1-2, Tables 1-2).

\section{VI}

Wilke, J.B.H.*, Hindermann, M., Berghoff, S.A., Zihsler, S., Arinrad, S., Ronnenberg, A., Barnkothe, N., Steixner-Kumar, A.A., Röglin, S., Stöcker, W., Hollmann, M., Nave, K.A., Lühder, F. and Ehrenreich, H. (2021). Autoantibodies agains NMDA receptor 1 modify rather than cause encephalitis. Molecular Psychiatry, 1-14.

Personal contributions: I contributed to the organization, acquisition and processing of all behavioral data (Figures 2 and 5, Tables 1-2).

* Shared first authorship 


\title{
Curriculum Vitae
}

\section{Sahab Arinrad}

\author{
26.10.1986. Teheran, Iran \\ Nonnenstieg 45, 37075 Göttingen \\ 畕 (+49) 1797599982 I $\square$ arinrad@em.mpg.de
}

\section{Academic career}

\section{Doctoral studies}

Georg-August-University Göttingen

Clinical Neuroscience, Max Planck Institute of Experimental Medicine

Prof. Dr. Dr. Hannelore Ehrenreich

Lower Saxony, Germany

\section{Master of Science studies}

Julius-Maximilians-University Würzburg

Department of Functional Neuroanatomy

Clinic and Polyclinic for Psychiatry, Psychosomatics and Psychotherapy

PD Dr. Angelika Schmitt-Böhrer

Bavaria, Germany

\section{Bachelor of Science studies}

University of Regensburg

Department of Behavioral and Molecular Neurobiology

Prof. Dr. Inga Neumann

Bavaria, Germany

\section{General qualification for university entrance}

Hainberg Grammar School Göttingen

Lower Saxony, Germany 


\section{Appendix III}

\section{List of publications}

1. Arinrad, S., Siems, S.B., Eichel, M.A., Depp, C., Ronnenberg, A., Hammerschmidt, K, Lüders, K.A., Werner, H.B., Ehrenreich, H. and Nave, K.A. (under revision). Isolated catatoniaexecutive dysfunction complex in aged mice induced by forebrain-specific loss of myelin integrity.

2. Arinrad, S.*, Wilke, J.B.*, Seelbach, A., Doeren, J., Hindermann, M., Butt, U.J., SteixnerKumar, A.A., Spieth, L., Ronnenberg, A., Pan, H., Berghoff, S.A., Hollmann, M., Lühder, F., Nave, K.A., Becher, K. and Ehrenreich, H. (accepted by Molecular Psychiatry, 2021). NMDAR1 autoantibodies amplify behavioral phenotypes of genetic white matter inflammation: A mild encephalitis model with neuropsychiatric relevance.

3. Wilke, J.B.H.*, Hindermann, M.*, Moussavi, A., Butt, U.J., Dadarwal, R., Berghoff, S.A., Sarcheshmeh, A.K., Ronnenberg, A., Zihsler, S., Arinrad, S., Hardeland, R., Seidel, J., Lühder, F., Nave, K.A, Boretius, S. and Ehrenreich, H. (2021). Inducing sterile pyramidal neuronal death in mice to model distinct aspects of gray matter encephalitis. Acta Neuropathologica Communications, $9(1), 1-19$.

4. Wilke, J.B.H, Hindermann, M., Berghoff, S.A., Zihsler, S., Arinrad, S., Ronnenberg, A., Barnkothe, N., Steixner-Kumar, A.A., Röglin, S., Stöcker, W., Hollmann, M., Nave, K.A., Lühder, F. and Ehrenreich, H. (2021). Autoantibodies agains NMDA receptor 1 modify rather than cause encephalitis. Molecular Psychiatry, 1-14.

5. Butt, U.J.*, Steixner-Kumar, A.A.*, Depp, C*, Sun, T., Hassouna, I., Wüstefeld, L., Arinrad, S., Zillmann, M., Schopf, N., Garcia-Agudo, L.F., Bode, U., Ronnenberg, A., Goebbels, S., Bonn, S., Katschinski, D.M., Nave, K.A. and Ehrenreich, H. (2021). Hippocampal neurons respond to brain activity with functional hypoxia. Molecular Psychiatry, 26:1790-1807.

6. Wakhloo, D.*, Scharkowski, F.*, Curto, Y.*, Butt, U.J., Bansal, V., Steixner-Kumar, A.A., Wüstefeld, L., Rajput A., Arinrad, S., Zillmann, M.R., Seelbach, A., Hassouna, I., Schneider, K., Ibrahim., A.Q., Werner., H.B., Martens, H., Miskowiak, K., Wojcik, S.M., Bonn, S., Nacher, J., Nave, K.A. and Ehrenreich, H. (2020). Functional hypoxia drives neuroplasticity and neurogenesis via brain erythropoietin. Nature communications, 11(1), 1-12.

7. Garcia-Agudo, L.F.*, Janova, H*., Sendler, L.E.*, Arinrad, S., Steixner, A.A., Hassouna, I., Balmuth, E., Ronnenberg, A., Schopf, N., van der Flier, F.J., Begemann, M., Martens, H., 
Weber, M.S., Boretius, S., Nave, K.A. and Ehrenreich, H. (2019). Genetically induced brain inflammation by Cnp deletion transiently benefits from microglia depletion. The FASEB Journal, 33(7), 8634-8647.

8. Janova, H.*, Arinrad, S.*, Balmuth, E.*, Mitjans, M., Hertel, J., Habes, M., Bittner, R.A., Pan, H., Goebbels, S., Begemann, M., Gerwig, U.C., Langner, S., Werner, H.B., Kittel-Schneider, S., Homuth, G., Davatzikos, C., Völzke, H., West, B.L., Reif, A., Grabe, H.J., Boretius, S., Ehrenreich, H. and Nave, K.A. (2018). Microglia ablation alleviates myelin-associated catatonic signs in mice. Journal of Clinical Investigation, 128(2):734-745.

9. Dere, E., Ronnenberg, A., Tampe, B., Arinrad, S., Schmidt, M., Zeisberg, E., \& Ehrenreich, H. (2018). Cognitive, emotional and social phenotyping of mice in an observer-independent setting. Neurobiology of learning and memory, 150, 136-150.

* Shared first authorship. 


\section{Appendix III}

\section{Conferences and lectures}

\section{Conference talk}

$08 / 2019$

Title: Assessing executive function in mice

Baltic Summer School: Behavioral Characterization of Rodent Models of Major Brain Disorders

University of Helsinki and University of Eastern Finland

Tartu, Estonia

\section{Poster presentation}

Title: Improving Cognition - A role for the brain erythropoietin system and bypoxia

Scientific Advisory Board - Max Planck Institute of Eperimental Medicine

Göttingen, Germany

\section{Lecture}

Title: Testing of schizophrenia-related functions in mice

Interdisciplinary Workshop: Translational Neuroscience "Schizophrenia"

Clinical Neuroscience, Max Planck Institute of Experimental Medicine

Göttingen, Germany

\section{References}

\section{Prof. Dr. Dr. Hannelore Ehrenreich}

Clinical Neuroscience, Max Planck Institute of Experimental Medicine, Göttingen (+49) 551 3899615, ehrenreich@em.mpg.de

\section{PD Dr. Angelika Schmitt-Böhrer}

Functional Neuroanatomy, Clinic and Polyclinic for Psychiatry, Psychosomatics and Psychotherapy, Würzburg

(+49) 931 20176402, schmitt_a3@ukw.de

\section{Prof. Dr. Inga Neumann}

Department of Behavioral and Molecular Neurobiology, University of Regensburg (+49) 941 9433055, inga.neumann@ur.de

\section{Prof. Dr. Oliver Bosch}

Department of Behavioral and Molecular Neurobiology, University of Regensburg (+49) 941 9433076, oliver.bosch@ur.de 


\section{Appendix}

\section{Professional qualifications}

\section{Behavioral characterization of mouse models of neuropsychiatric disorders}

Neonatal assessment, pre-/postnatal stress paradigms, locomotion and exploration, anxiety-

/depression-like behavior, anhedonia, motor performance and coordination, sensory performance, sociability and social memory, vocalization, nociception, sensorimotor gating, fear conditioning, catatonia and cognition (working memory, place/reversal learning, episodic-like memory, cognitive flexibility, executive function), FELASA B qualification, preparation of ethical proposals.

\section{Surgical interventions and dissections}

Anaesthesia, i.p./i.c.v./tail vein injections, transcardial perfusions, stereotactic placement of cannulae, hippocampus, CA1, amygdala, hypothalamus and prefrontal cortex.

\section{Molecular biology}

RT-qPCR, western blot, RNA/DNA extraction, immunohistochemistry.

\section{Computer literacy}

GraphPad Prism, SPSS, MBF Bioscience Stereoinvestigator, JWatcher, BioRad CFX Manager, MS Office, Adobe Photoshop and Illustrator, Endnote Bibliography, Mendeley Bibliography.

\section{Languages}

English, German, Farsi, Spanish. 